\title{
Wirkungsmonitoring der FFG Förderung 2015
}


Diese Studie wurde im Auftrag der Österreichischen Forschungsförderungsgesellschaft (FFG) durchgeführt.

\section{VERFASSER DES BERICHTS:}

Peter Kaufmann

Laurenz Wolf

INTERNES REVIEW:

Sonja Sheikh

\section{LAYOUT:}

Susanne Fröhlich/Martina Gugerell

Die vorliegende Studie wurde nach allen Maßstäben der Sorgfalt erstellt.

Die KMU Forschung Austria übernimmt jedoch keine Haftung für Schäden oder Folgeschäden, die auf diese Studie oder auf mögliche fehlerhafte Angaben zurückgehen.

Dieses Werk ist urheberrechtlich geschützt. Jede Art von Nachdruck, Vervielfältigung, Verbreitung, Wiedergabe, Übersetzung oder Einspeicherung und Verwendung in Datenverarbeitungssystemen, und sei es auch nur auszugsweise, ist nur mit ausdrücklicher Zustimmung des Auftraggebers gestattet.

Mitglied bei:

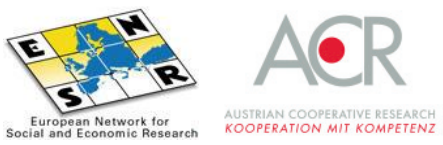




\section{Inhaltsverzeichnis}

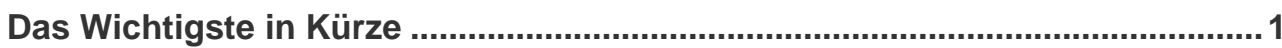

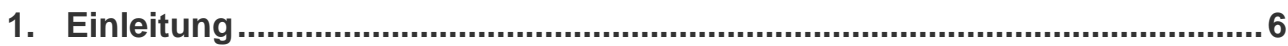

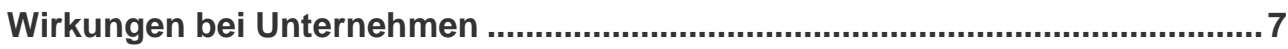

2. Methodik und Eckdaten der analysierten Unternehmen............................7

3. Positionierung der Projekte im Portfolio der Unternehmen ........................14

4. Wirtschaftliche Projektergebnisse …........................................................ 19

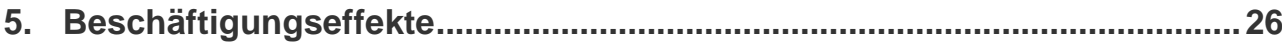

6. Auswirkungen auf die Forschungs-, Entwicklungs-, und Innovationsaktivitäten der Unternehmen .....................................................30

7. Rolle von ,Open Innovation‘ im Innovationsprozess ..................................40

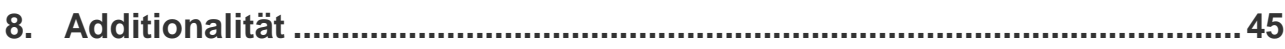

Wirkungen bei Forschungseinheiten .................................................................. 48

9. Methodik und Eckdaten der analysierten Forschungseinheiten ...............48

10. Initiierung und Positionierung der Projekte im Portfolio der Forschungseinheiten..................................................................................... 51

11. Effekte auf die Forschungseinheiten und Spillovers ................................57

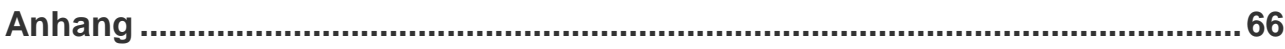




\section{Tabellenverzeichnis}

Tabelle 1 Verteilung der in die Analyse einbezogenen und 2011

abgeschlossenen Projektbeteiligungen durch Unternehmen

Tabelle 2 Zuordnung der Programme und erfassten Unternehmensfragebögen zu FFG Förderungs- und Finanzierungsinstrumenten

Tabelle 3 Verteilung der 2011 abgeschlossenen Projekte nach Beschäftigtengrößenklassen der Unternehmen, Anzahl

Tabelle 4 Verteilung der analysierten und 2011 abgeschlossenen Projekte nach ÖNACE-Klassifikation der Projektteilnahmen.

Tabelle 5 Verteilung der 2011 abgeschlossenen Projekte nach der Rolle des Projekts hinsichtlich der FuE Aktivitäten im Unternehmen.....

Tabelle 6 Erreichung des Projektzieles aus technischer und wirtschaftlicher Sicht nach Unternehmensgrößenklassen, 2011 abgeschlossene Projekte.

Tabelle 7 Projektkosten nach Förderungs- und Finanzierungsinstrumenten sowie Unternehmensgröße.

Tabelle 8 Wirtschaftliche Verwertung der vor 4 Jahren abgeschlossenen Projekte.

Tabelle 9 Direkte wirtschaftliche Ergebnisse der im Jahr 2011 abgeschlossenen Projekte, in Mio. $€$ 24

Tabelle 10 Externe und interne Rekrutierung von Forschungspersonal ..............28

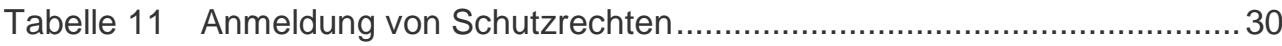

Tabelle 12 Arten der Innovation von im Jahr 2011 abgeschlossenen Projekten

Tabelle 13 Effekte auf Netzwerkbildung, 2011 abgeschlossene Projekte 37

Tabelle 142011 abgeschlossene Projekte; Rücklauf der online befragten Forschungseinheiten nach Programmbeteiligung ....

Tabelle 152011 abgeschlossene Projekte; Rücklauf der online befragten Forschungseinheiten nach Organisationsart ..................................50

Tabelle 162011 abgeschlossene Projekte; Instrumentenzuordnung ..................50

Tabelle 18 Verortung der Projekte im Institutsprofil .........................................53

Tabelle 19 Verteilung der am Projekt beteiligten FuE-MitarbeiterInnen...............54

Tabelle 20 Gewerbliche Schutzrechte unter Teilnahme der Forschungs-

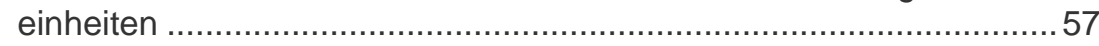

Tabelle 21 Anzahl und Art der Folgeprojekte und deren Finanzierung ................60

Tabelle 22 KMU-Definition der Europäische Kommission.....................................66

Tabelle 23 Durchschnittliche Gesamtkosten und Förderbarwerte nichtantwortender Unternehmen

Tabelle 24 Frage 7: Wurde das Projektziel aus technischer / wirtschaftlicher Sicht erreicht? Im Bundesländervergleich 
Tabelle 25 Frage 10: Werden die Projektergebnisse im Unternehmen wirtschaftlich verwertet? Im Bundesländervergleich ......................... 70

Tabelle 26 Frage 22: Sind neue Kontakte/Kooperationen entstanden? ..............71

Tabelle 27 Frage 23: Wurden Kontakte nach Projektabschluss weitergeführt?

Tabelle 28 Frage 7: Wurde das Projektziel aus technisch/wirtschaftlicher Sicht erreicht? Nach Beschäftigtengrößenklassen .............................. 72

Tabelle 29 Frage 10: Werden die Projektergebnisse im Unternehmen wirtschaftlich verwertet? Nach Beschäftigtengrößenklassen ............. 73

Tabelle 30 Frage 22: Sind neue Kontakte/Kooperationen entstanden? ...............74

Tabelle 31 Frage 23: Wurden Kontakte nach Projektabschluss weitergeführt?

Tabelle 32 Frage 7: Wurde das Projektziel aus technisch/wirtschaftlicher Sicht erreicht? Nach ÖNACE Klassifikation der Projekte

Tabelle 33 Frage 10: Werden die Projektergebnisse im Unternehmen wirtschaftlich verwertet? Nach ÖNACE Klassifikation der

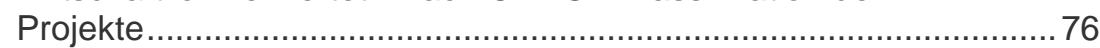

Tabelle 34 Frage 22: Sind neue Kontakte/Kooperationen entstanden? ..............77

Tabelle 35 Frage 23: Wurden Kontakte nach Projektabschluss weitergeführt? 


\section{Abbildungsverzeichnis}

Abbildung 1 Anteile des Basisprogramms und anderer Programme in den letzten fünf Jahren

Abbildung 2 Verteilung der 2011 abgeschlossenen Projekte nach der Rolle des Projekts im Portfolio des Unternehmens

Abbildung 3 Erreichung der Projektziele aus technischer und wirtschaftlicher Sicht, 2011 abgeschlossene Projekte .....

Abbildung 4 Gründe für wirtschaftliche Zielverfehlung ................................... 17

Abbildung 5 Wirtschaftliche Verwertung der vor 4 Jahren abgeschlossenen Projekte

Abbildung 6 Wirtschaftliche Verwertung der vor 4 Jahren abgeschlossenen Projekte nach Förderungs- und Finanzierungsinstrumenten ........22

Abbildung 7 Fördervolumen der abgeschlossenen Projekte mit bzw. ohne wirtschaftlicher Zielerreichung aus den FFG-geförderten Projekten, nach Größenklassen und Form der Zuwendung ..........23

Abbildung $8 \quad$ Fördermultiplikator im Zeitverlauf ................................................25

Abbildung 9 Beschäftigungseffekte der im Jahr 2011 abgeschlossenen Projekte nach Beschäftigtengrößenklassen der Unternehmen, Anzahl nach Köpfen

Abbildung 10 Durchschnittlicher Barwert der Fördermittel je gesicherten und geschaffenen Arbeitsplatz der in den Jahren 2007 bis 2011 abgeschlossenen Projekte, nach MA-Größenklassen ...................29

Abbildung 11 Wissenschaftliche Publikationen nach Instrumenten .....................31

Abbildung 12 Arten der Innovation von im Jahr 2011 abgeschlossenen Projekten

Abbildung 13 Risiken in der Entwicklung von Dienstleistungsinnovationen.........33

Abbildung 14 Inanspruchnahme zusätzlicher Förderangebote ...........................34

Abbildung 15 Auswirkungen der 2011 abgeschlossenen Projekte auf unterschiedliche Bereiche im Unternehmen.

Abbildung 16 Veränderung der technologischen Wettbewerbsposition ................36

Abbildung 17 Form der Weiterführung von neuen und bestehenden

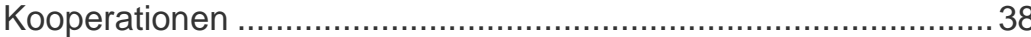

Abbildung 18 Kategorisierung von Open Innovation Prozessen .........................40

Abbildung 19 Impulse zur Öffnung von Innovationsprozessen ...........................41

Abbildung 20 Einbindung externer Partner in den Innovationsprozess .............. 42

Abbildung 21 Planen die Unternehmen den Innovationsprozess künftig stärker zu öffnen? ................................................................... 43

Abbildung 22 Hätten Sie das Projekt auch ohne Förderung durchgeführt? ........ 45

Abbildung 23 Additionalität der Projektteilnahmen mit Projektende 2007-2011.

Abbildung 24 Woher kam der Impuls für das Projekt 
Abbildung 25 Zuordnung zu unmittelbaren FuE-Vorprojekten ...........................52

Abbildung 26 Größe der involvierten FuE-MitarbeiterInnenteams ......................54

Abbildung 27 Inhaltliche Projektzielerreichung ...................................................55

Abbildung 28 Wie erfolgreich stufen Sie die FuE Kooperation im konkreten Projekt ein? ......................................................................... 56

Abbildung 29 Wie beurteilen Sie die Umsetzungs- und Nutzungsmöglichkeiten der Projektergebnisse? ...................................................58

Abbildung 30 Welche der folgenden Effekte ergaben sich aufgrund des FFG-Projekts?

Abbildung 31 Welche der folgenden Aktivitäten zum Transfer der Projektergebnisse wurden von Ihnen gesetzt? 61

Abbildung 32 Häufigkeiten der Disseminationsergebnisse ...............................62

Abbildung 33 Welche weiteren Transferaktivitäten führten Sie durch?................63

Abbildung 34 Wie schätzen Sie die Wirkung des Projekts auf Ihr Institut ein? ...64

Abbildung 35 Wie häufig führt Ihr Institut private Auftragsforschung bzw. Beratungsleistungen direkt für Unternehmen oder Institutionen durch? 


\section{Das Wichtigste in Kürze}

Das Wirkungsmonitoring der im Jahr 2011 abgeschlossenen FFG Projekte bezieht sich einerseits auf geförderte Projekte, in denen Unternehmen als Projektträger oder Partner auftreten. Darüber hinaus werden die im Vorjahr erstmals untersuchten, beteiligten Forschungseinheiten (inkl. Hochschulen) nun dauerhaft in die Befragung aufgenommen.

Die heuer erstmals durchgeführte Auswertung nach Förderungs- und Finanzierungsinstrumenten der FFG brachte einige interessante Detailergebnisse, die sich in den nächsten Jahren aber noch bestätigen müssen.

\section{Wirkungen bei Unternehmen}

Die traditionell hohen Rücklaufquoten der Vorjahre konnten in diesem Jahr mit einer Netto-Rücklaufquote von $75 \%$ sogar übertroffen werden. Dies lässt sich auf eine Kombination aus elektronischer und postalischer Befragung zurückführen, die im Zuge des Pilotversuchs einer online-Befragung umgesetzt wurde.

Es konnte auch der Höchststand des Erhebungsjahres 2013 (498 Projektteilnahmen durch 406 Unternehmen) mit 548 Projektteilnahmen durch 459 Unternehmen übertroffen werden. Es setzte sich der Trend der letzten Jahre insofern fort, als vermehrt Projekte über das Basisprogramm hinaus erfasst wurden. Heuer stellen diese mit $56 \%$ erstmals die Mehrheit dar.

Insgesamt lässt sich festhalten, dass die Komplexität (insb. Größe der Konsortien) sowie die Anwendungsnähe der Projekte auch vier Jahre nach Projektende die bestimmenden Faktoren darstellen, inwieweit zum gegenwärtigen Zeitpunkt Wirkungen nachgewiesen werden können.

87\% der Unternehmen nutzten die Projekte für den Ausbau von bereits bestehenden FuE-Aktivitäten. Für 10\% stellte das geförderte Projekt die erste FuE-Tätigkeit dar. Für die Mehrheit dieser Unternehmen, d.h. 7\%-Punkte (bzw. rd. 70\% der FuENeulinge) wirkte das Projekt als Anstoß für weitere FuE-Projekte.

Mit 35\% der Projekte wollten die Unternehmen in für sie neue Geschäfts- bzw. Aktivitätsfelder vorstoßen. Zwischen den einzelnen FFG Instrumenten lassen sich tlw. deutliche Unterschiede feststellen. Am häufigsten wurden die BRIDGE Projekte und Markteinführungsprojekte durch die Unternehmen als Bausteine bestehender Aktivitätsfelder angesehen, während Innovationsnetzwerke vergleichsweise häufiger ein neues Aktivitätsfeld angestoßen haben.

Einen Projekterfolg aus technischer Sicht im Sinne davon, dass die Ergebnisse nutzbringend eingesetzt werden konnten, verzeichneten 91\% der befragten Unternehmen. Darüber hinaus konnten mehr als die Hälfte der geförderten Projekte (56\%) 
auch die wirtschaftlichen Projektziele erreichen. Diese Erfolgsquote ist insbesondere im Basisprogramm von sehr hoher Beständigkeit (rd. 60\%).

Die Projektteilnahmen trugen nach Einschätzung der Unternehmen zu 16\% dazu bei, dass betreffend der konkreten Technologie international zur Spitze aufgeschlossen werden konnte. Überdurchschnittlich gelang dies Unternehmen, die im Instrument Einzelprojekt aktiv waren. Zumindest eine Verbesserung der technologischen Wettbewerbsposition wurde bei rd. 70\% aller Projektteilnahmen gesehen

In den ersten vier Jahren nach Projektende entfalteten 48\% der Projektbeteiligungen positive Wirkungen auf die Erhaltung und Schaffung von Arbeitsplätzen (2014: 50\%, 2013: 52\%). Dieser leichte Rückgang ist auf den höheren Anteil von Projekten jenseits der Einzelprojekte zurückzuführen. Beschäftigungseffekte bei FuEMitarbeiterInnen ergaben sich bei $57 \%$ aller Projektbeteiligungen. Diese sind auf externe und interne Rekrutierung zurückzuführen.

Insgesamt führten 84\% der Projektteilnahmen zu zumindest einer Innovation. Die Art der Innovationen verteilt sich über die Jahre mit einer bemerkenswerten Konstanz, wenngleich der Anteil von neuen Produkten in den letzten Jahren etwas rückläufig ist, und jener von organisatorischen Innovationen leicht steigend. Dies ist wiederum allein auf den höheren Anteil von Nicht-Basisprogramm-Projekten zurückzuführen.

Schutzrechte wurden in $24 \%$ der untersuchten Projekte eingereicht, wobei diese eine Summe von 512 Schutzrechten bilden. Diese konzentrieren sich stark auf einzelne Projekte (insb. Einzel- und Leitprojekte), in denen oft Patentfamilien (von Großunternehmen) eingereicht wurden. Im Instrument Einzelprojekt (BP) wurden durchschnittlich 1,28 Schutzrechte je Projekt (mit und ohne Projektpartner) angemeldet, Kooperationsprojekt: 0,82, Leitprojekt: 2,13, Wissenschaftstransfer: 0,24, Innovationsnetzwerk: 0,27.

Die Forschungsaktivitäten der befragten Unternehmen trugen dazu bei, dass $78 \%$ der Projektteilnahmen (425) zu neuen Kontakten führten bzw. bestehende Kontakte intensiviert wurden. Neue bzw. intensivierte Kontakte haben die Einzelprojekte v.a. im Bereich der universitären und außeruniversitären Bereich geknüpft sowie mit Zulieferern und Kunden. Gleiches gilt, etwas verstärkt auf Ebene der Forschungseinrichtungen, für die Kooperationsprojekte.

46\% der Projektteilnahmen wurden von ,Neukunden' (definiert als erstes FFG Projekt zwischen 2007 und 2010) durchgeführt. Wie auch in den letzten Jahren zeigt sich, dass Neukunden einen relativ höheren Nutzen hinsichtlich der „weichen“ Innovationsfaktoren aus den Projekten ableiten (neue Kooperation, etc.), aber hinsichtlich der kommerziellen Verwertung der Forschungsergebnisse hinter den Bestandskunden liegen, die mehr Erfahrung mit derartigen Projekten aufweisen. 
Insgesamt wären 31\% der Projekte ohne Förderung nicht durchgeführt worden (Einzelprojekte: 18\%). Dieser Anteil hat sich über die Jahre erhöht und deutet darauf hin, dass in den Nach-Krisenjahren geringere Mitnahmeeffekte vorliegen. Instrumente mit höherer Marktferne bzw. Risiken zeigen höhere Additionalität der Förderung. Inwieweit dies auf die konjunkturelle Entwicklung der letzten Jahre bzw. administrative Aspekte innerhalb der FFG zurückzuführen ist, kann leider nicht getrennt dargestellt werden. Aus den restlichen Teilen des Wirkungsmonitorings können wir jedoch ableiten, dass sich die Unternehmen aufgrund des wirtschaftlichen Umfeldes nach der Wirtschaftskrise nach wie vor etwas schwer tun, mit ihren Innovationen Umsatzvolumina in der Höhe des Vor-Krisenniveaus zu erreichen. Dies belastet natürlich auch die FEl-Aktivitäten der Unternehmen; in solchen Zeiten steigt die Relevanz der Aktivitäten von Innovationsagenturen über das normale Maß.

Aufgrund des einmalig gestellten Fragenblocks zum Thema ,Open Innovation' stellen wir fest, dass dies bei FEl-aktiven Unternehmen durchaus, wenn auch selektiv, auf Interesse stößt. Die Neigung zur Öffnung des Innovationsprozesses hängt von strukturellen Markmalen des Unternehmens ab (eher kleinere Unternehmen), der Branche bzw. dem Technologiefeld, in dem das Unternehmen tätig ist, sowie der Zielmärkte und damit zusammenhängend der Intensität der Konkurrenzsituation bzw. der Kundennähe.

\section{Wirkungen bei Forschungseinheiten}

Das Wirkungsmonitoring wurde dieses Jahr zum zweiten Mal auf Forschungseinheiten (FE, inkl. Hochschulen) ausgeweitet, und mittels eines Online-Fragebogens umgesetzt. Von den 403 erreichten Projektteilnehmern beantworteten 192 den Fragebogen; dies entspricht einer Netto-Rücklaufquote von $48 \%$.

Der Schwerpunkt der FE-Befragung liegt nach der Struktur der Forschungs- und Finanzierungsinstrumente der FFG bei den Kooperationsprojekten (TP und ALR, $68 \%$ ), gefolgt von Wissenschaftstransfer (BRIDGE, 15\%), sowie Strukturaufbau und Innovationsnetzwerke (SP, je 6\%). Im Unterschied zur Unternehmensbefragung nimmt das Basisprogramm (Instrument Einzelprojekt) mit 4\% nur eine untergeordnete Rolle ein.

Der Impuls zur Durchführung der Projekte ist in knapp 46\% der Fälle von den FE selbst ausgegangen. Insgesamt zeigen sich die FE heuer etwas initiativer (Vorjahr: $39 \%$ ). Dies ist hauptsächlich auf den höheren Anteil von Projekten aus dem Programm Neue Energien zurückzuführen, die mit rd. $70 \%$ Projektideen aus dem eigenen Forschungsinstitut umsetzten.

Wie im Vorjahr konnten $61 \%$ der Projekte inhaltlich zumindest einem FuE-Vorprojekt in der FE zugeordnet werden. Im Bereich TP wurden rd. 40\% der Projekte ohne unmittelbare Vorprojekte gestartet. Im restlichen FFG Portfolio ist der Anteil deutlich geringer. 
$78 \%$ der inhaltlich zurechenbaren Vorprojekte wurden durch die FFG unterstützt. In $30 \%$ der Fälle wurden Mittel der Europäischen Kommission, Landesförderungen, oder des FWF in Anspruch genommen. Bei 57\% der Projektbeteiligungen sind bislang Folgeprojekte entstanden. Rd. 43\% der Projektteilnahmen werden wieder durch die FFG unterstützt. Niedriger ist der Anteil direkt durch Unternehmen (u.a. Institutionen) unterstützter Projekte (21\%), anderer Förderprogramme auf nationaler und regionaler Ebene (13\%), bzw. EU Projekte (14\%).

In 57\% aller 163 Forschungsprojekte (93 Projekte $=114$ Projektteilnahmen) gab es eine Festlegung der Verwertungsrechte (in Form von Konsortialverträgen oder Absprachen). Trotzdem sind eingetragene Schutzrechte bei Forschungsinstituten eher die Ausnahme. Von diesen 114 Projektteilnehmern waren in 15\% der Fälle FE an den Schutzrechten beteiligt.

Von diesen 114 Verwertungsrechten waren in 15\% der Fälle Forschungseinheiten an den Schutzrechten beteiligt. Auf alle Projektbeteiligungen bezogen sind es 9\%.

Es wurden 76 weitere Anwendungsgebiete für die Projektergebnisse angesprochen, manche davon von sehr generischer Natur. Damit stellt sich die Frage nach effektiven Diffusions- bzw. Transfermechanismen von Projektergebnissen, da davon auszugehen ist, dass ein hoher Anteil davon von den beteiligten Instituten und auch Unternehmen nicht weiterverfolgt wird. Die Antwort dazu sollte im Zusammenhang mit den unterschiedlichen Formen der Öffnung von Innovationsprozessen bzw. der Resultate gesucht werden (Stichworte: Open Innovation und Open Access).

Nach außen gerichtete Wirkungen der Projekte werden primär durch erweiterte Expertise, Reputation und Netzwerkeffekte festgemacht. Über 90\% der Forschungseinheiten gestanden dem Projekt zu, die Expertise des Instituts erweitert zu haben. Rund vier Fünftel der Forschungsvorhaben führten zu einer erhöhten Attraktivität als Kooperationspartner und zur erhöhten Sichtbarkeit der Forschungseinrichtungen in der Zielgruppe. Auch die Erweiterung des Forschungsnetzwerks bzw. die Weiterverfolgung von anwendungsorientierten Fragen durch das Projekt wurden häufig als sehr zutreffend beurteilt. Neue Lehrinhalte sind natürlich nur für lehrende Forschungseinheiten relevant; hier ergeben sich jedoch deutliche Wechselwirkungen zwischen Lehre und Forschung.

Der Wechsel von Forschungspersonal nach dem Projektende (inkl. Diplomandlnnen und DissertantInnen) in die am Projekt beteiligten Unternehmen liegt bei 16\% (Vorjahr: 17\%); 7\% wechselten zu einem wissenschaftlichen Projektpartner (Vorjahr: 8\%). Wie auch im Vorjahr verblieb die Hälfte bei der Forschungseinheit und wurden dort weiterbeschäftigt. Dies summiert sich auf 217 Personen, die an der FE weiterbeschäftigt wurden. 
Drei Viertel der Projektteilnahmen führten zu neuen Kooperationen und Kontakten im FuE Bereich. Im Vorjahr waren dies noch zwei Drittel, da ein geringerer Anteil an Kooperationsprojekten enthalten war. Wieder rd. die Hälfte der Befragten gab an, dass weiterführende Diplomarbeiten oder Dissertationen angestoßen wurden. Dies summiert sich auf rd. 140 Diplomarbeiten und Dissertationen, die noch nach Abschluss der Projekte gestartet wurden.

Für die Erfüllung der FuE-Tätigkeiten wurden von 118 Forschungseinrichtungen 971 MitarbeiterInnen zur Bearbeitung der Aufgabenstellungen einbezogen. Mehr als die Hälfte davon war Forschungspersonal auf der Ebene von Post-docs, LabormitarbeiterInnen, DozentInnen, etc. Des Weiteren waren 254 Diplomandlnnen (in 107 Projekten) und 204 Doktorandlnnen (in 111 Projekten) in die FuE-Tätigkeiten eingebunden.

Die mit rd. 79\% am häufigsten gewählte Art der Dissemination der Projektergebnisse war zum einen Publikationen in akademisch referierten Journalen und Konferenzen. Aber auch akademische Konferenzen (rd. 69\%) und praxisorientierte Fachzeitschriften $(57 \%)$ werden genutzt.

In Summe belaufen sich die Disseminationstätigkeiten auf 958 gesetzte Maßnahmen (durchschnittlich 5,0 je Projektteilnahme). Davon sind 314 referierte Publikationen mit, und 179 Publikationen ohne Kooperationspartner. Diese 493 Publikationen verteilen sich auf 104 Projektteilnahmen. 


\section{Einleitung}

Das Wirkungsmonitoring des Jahres 2015 bezieht sich auf geförderte Projekte, die im Jahr 2011 abgeschlossen wurden. Die empirische Basis stellt eine standardisierte Befragung von Fördernehmern dar, die mit Daten der FFG Projektdatenbank ergänzt wurden. Indem die Analyse auf Projekte vier Jahre nach dem formalen Projektende basiert, sollen Aussagen über die mittelfristigen Auswirkungen der eingesetzten Fördermittel ermöglicht werden.

Die Ausweitung der Analyse über Unternehmen hinaus auf die beteiligten Forschungseinheiten (Hochschulen, Forschungseinrichtungen) wurde im letzten Jahr erprobt, und im heurigen Berichtsjahr als dauerhafter Bestandteil des Berichts etabliert.

Ab dem Jahr 2010 (Projektende 2006) wurde das Wirkungsmonitoring der Unternehmen über das Basisprogramm hinaus auf weitere Programme der FFG ausgeweitet, sofern nicht aufgrund bestimmter Eigenheiten des Programms bereits ein eigenes Wirkungsmonitoring bestand (letzteres gilt für Zentren-Programme) oder die typischen Projekte Förderbarwerte unter zwanzigtausend Euro aufwiesen (Innovationsschecks, Feasibility Studien, Anbahnungsfinanzierungen, etc.).

In den letzten Jahren ist der Anteil an Projekten außerhalb des Basisprogramms kontinuierlich gestiegen und stellt heuer erstmals die Mehrheit dar. Damit ist eine differenziertere Auswertung möglich, die erstmals hauptsächlich anhand der Förderungs- und Finanzierungsinstrumente der FFG vorgenommen wird.

An dieser Stelle soll darauf hingewiesen werden, dass anhand des Analysedesigns keine quantitative Erfassung der Nettoeffekte möglich ist, da sich schwer fassbare Nebeneffekte (Verdrängung, Substitution, etc.) positiv wie auch negativ auf die hier ermittelten Resultate auswirken können. Das jährlich umgesetzte Wirkungsmonitoring legt den Schwerpunkt auf die Analyse von deskriptiven und qualitativen Inhalten und analysiert diese im Detail, um ein umfassenderes Bild potenzieller Wirkungen auf die teilnehmenden Unternehmen und Forschungseinheiten zu erhalten. Eine analytisch-quantitative Schätzung von Input-, Verhaltens-, und Output-Additionalität wurde im Jahr 2004 für das Basisprogramm vorgenommen, die im Wesentlichen die positiven Ergebnisse des Wirkungsmonitorings über die Jahre bestätigen konnte (Schibany et al. 2004. Evaluation FFF - impact analysis. Background report 3.2., INTEREG research report series, Vienna; und Falk 2004. Behavioural additionality effects of R\&D-subsidies - empirical evidence from Austria. Austrian Institute of Economic Research). 


\section{Wirkungen bei Unternehmen}

\section{Methodik und Eckdaten der analysierten Unternehmen}

Im Zuge der Umstellung auf eine Online-Erhebung wurde der Fragebogen den Unternehmen erstmals elektronisch übermittelt. Die Kontaktadressen (ProjektleiterInnen, kaufmännische AnsprechpartnerInnen) wurden von der FFG zur Verfügung gestellt. Fehlende Daten wurden von der KMU Forschung Austria nacherfasst. Damit konnte jedoch ein relativ hoher Anteil an Kontakten in den Unternehmen nicht direkt angesprochen werden. Daher wurde, wie in den vergangenen Jahren, zusätzlich auch die Unternehmensführung mittels einer postalischen Aussendungen angeschrieben. Dies führte wiederum zu den gewohnten sehr hohen Rücklaufquoten. Die beantworteten Fragebögen sind direkt bei der KMU Forschung Austria eingegangen. Als ein Resultat dessen, sollten in Zukunft auch der Online-Fragebogen an die Geschäftsführung versendet werden, damit diese die relevante Person innerhalb des Unternehmens zur Beantwortung identifizieren kann.

Die diesjährige Unternehmensbefragung beinhaltet auch eine Sonderbefragung zum Umgang mit dem Thema Open Innovation.

Da Unternehmen mehrere Projekte zeitgleich durchführen können, ist die Zahl der verwertbaren Fragebögen mit 548 höher als die Zahl der 459 geförderten Unternehmen. Des Weiteren können Projekte auch in Form von Kooperationen abgewickelt werden. Deshalb können Wirkungen auf mehrere Fördernehmer verteilt sein, und Angaben zu demselben Projekt erfolgen aus dem Blickwinkel des jeweils eigenen Unternehmens bzw. des eigenen Projektteils um Doppelzählungen zu vermeiden.

Der Querschnittscharakter der Befragung könnte insofern Verzerrungen beinhalten, als größere Innovationen oft in „Projektfamilien“ vorangetrieben werden, im Rahmen derer konsekutive Projekte mit unterschiedlichen Schwerpunkten und Finanzierungsstrukturen umgesetzt werden. Ein klassischer Fall ist z.B. dass ein Kleinunternehmen aufgrund eines singulären von der FFG geförderten Projekts eine Innovation am Markt lanciert. Am anderen Ende des Spektrums führt ein technologieorientiertes globales Unternehmen laufend mehrere FEI Projekte parallel durch. Dabei wird eine Zuordnung von wirtschaftlichen Effekten zu einem FFG Projekt oft schwierig. Dies ist auch der Grund dafür, dass Großunternehmen öfters keine konkret quantifizierten wirtschaftlichen Effekte im Rahmen des Wirkungsmonitorings angeben. Trotzdem kann es vorkommen, dass das geförderte Projekt eines Großunternehmens eine Kerntechnologie hinsichtlich der Produktentwicklung betrifft, mit der fallweise ein hoher Umsatz am Markt erwirtschaftet werden kann. Aus diesem Grund 
wird bei Unternehmen, die sehr hohe wirtschaftliche Effekte im Rahmen des Wirkungsmonitorings angeben, der Zusammenhang mit der FFG Förderung mit der KMU Forschung Austria am Telefon noch erörtert. Werte mit zu geringem kausalem Zusammenhang mit der Förderung wurden nicht in die Analyse einbezogen.

Im Jahr 2011 wurden 1.096 Projektbeteiligungen von Unternehmen abgeschlossen, die It. FFG Datenbank auch 2015 noch aktiv waren. Hiervon wurden Projektbeteiligungen mit einem Volumen unter $€ 20.000$,- sowie reine Studien abgezogen. Schließlich wurden 733 Fragebögen an noch bestehende, inländische Unternehmen versendet (mit mehrmaliger elektronischer Erinnerung bzw. postalischen Anschreiben), welche in 548 verwertbaren Rückmeldungen mündeten.

\section{Sehr hohe Rücklaufquote}

Die Fördernehmer des Basisprogramms (inkl. Competence Headquarters, Hightech Startups und Dienstleistungsinnovationen) stellen mit $44 \%$ die größte Gruppe der Antwortenden dar, und weisen mit einer Rücklaufquote von rd. 80\% eine überdurchschnittlich hohe Auskunftsfreudigkeit auf (Tabelle 1). Dies basiert auf den dahinterliegenden administrativen Aufwand durch wiederholte Ansprache, der heuer zu einer insgesamt sehr hohen Rücklaufquote von $75 \%$ führte.

Die auffallend niedrigen Rücklaufquoten in den Programmen NANO und KIRAS sind bei ersterem auf die niedrige Fallzahl zurückzuführen. Darüber hinaus ist eine gewisse „Antwortmüdigkeit" seitens der Unternehmen auf Grund mehrerer vorangegangener Befragungen im Zuge der begleitendenden Evaluierung zum KIRAS Programm naheliegend.

Thematische Programme (insb. Neue Energien und Mobilitätsprogramme), aber auch das Strukturprogramm COIN mit zunehmender Bedeutung

Die Zahl und der Anteil der Projektteilnahmen abseits des Basisprogramms sind, wie in den Vorjahren, weiter angestiegen. Erstmals stellen diese die Mehrheit dar (56\% inkl. BRIDGE und EUROSTARS). Wurde mit Projektende 2007 mit 89\% noch vorwiegend das Basisprogramm hinsichtlich seiner Wirkungseffekte untersucht, so wurden im Vorjahr (Projektende 2010) noch 55\% der Projektteilnahmen dem Basisprogramm zugeordnet (heuer: 44\%). 
Tabelle 1 Verteilung der in die Analyse einbezogenen und 2011 abgeschlossenen Projektbeteiligungen durch Unternehmen

\begin{tabular}{|c|c|c|c|c|c|}
\hline $\begin{array}{l}\mathrm{Be}- \\
\text { reich }\end{array}$ & Programm & $\begin{array}{l}\text { Ver- } \\
\text { sendet }\end{array}$ & $\begin{array}{l}\text { Ausge- } \\
\text { wertet }\end{array}$ & $\begin{array}{l}\text { Netto- } \\
\text { Rücklauf }\end{array}$ & $\begin{array}{l}\text { Anteil an ausgewer- } \\
\text { teten Fragebögen }\end{array}$ \\
\hline \multirow{3}{*}{$\mathrm{BP}$} & Basisprogramm & 304 & 242 & $80 \%$ & $44 \%$ \\
\hline & BRIDGE & 33 & 25 & $76 \%$ & $5 \%$ \\
\hline & EUROSTARS & 5 & 4 & $80 \%$ & $1 \%$ \\
\hline \multirow{10}{*}{ TP } & Neue Energien 2020 & 112 & 89 & $79 \%$ & $16 \%$ \\
\hline & IV2S, IV2Splus & 85 & 58 & $68 \%$ & $11 \%$ \\
\hline & FIT-IT & 39 & 30 & $77 \%$ & $5 \%$ \\
\hline & TAKE OFF & 25 & 18 & $72 \%$ & $3 \%$ \\
\hline & Energie d. Zukunft & 18 & 12 & $67 \%$ & $2 \%$ \\
\hline & benefit & 12 & 10 & $83 \%$ & $2 \%$ \\
\hline & AT:net & 13 & 7 & $54 \%$ & $1 \%$ \\
\hline & KIRAS & 16 & 6 & $38 \%$ & $1 \%$ \\
\hline & NANO & 6 & 3 & $50 \%$ & $1 \%$ \\
\hline & NAWI & 2 & 0 & $0 \%$ & $0 \%$ \\
\hline SP & COIN & 53 & 37 & $70 \%$ & $7 \%$ \\
\hline ALR & ASAP & 10 & 7 & $70 \%$ & $1 \%$ \\
\hline \multicolumn{2}{|c|}{ Gesamt } & 733 & 548 & $75 \%$ & $100 \%$ \\
\hline
\end{tabular}

Quelle: KMU Forschung Austria; BP = Bereich Basisprogramme, TP = Thematische Programme, $\mathrm{SP}=$ Strukturprogramme, $\mathrm{ALR}=$ Agentur für Luft- und Raumfahrt

Die räumliche Verteilung der Fördernehmer ist dem Anhang zu entnehmen. 
Abbildung 1 Anteile des Basisprogramms und anderer Programme in den letzten fünf Jahren (Unternehmen)

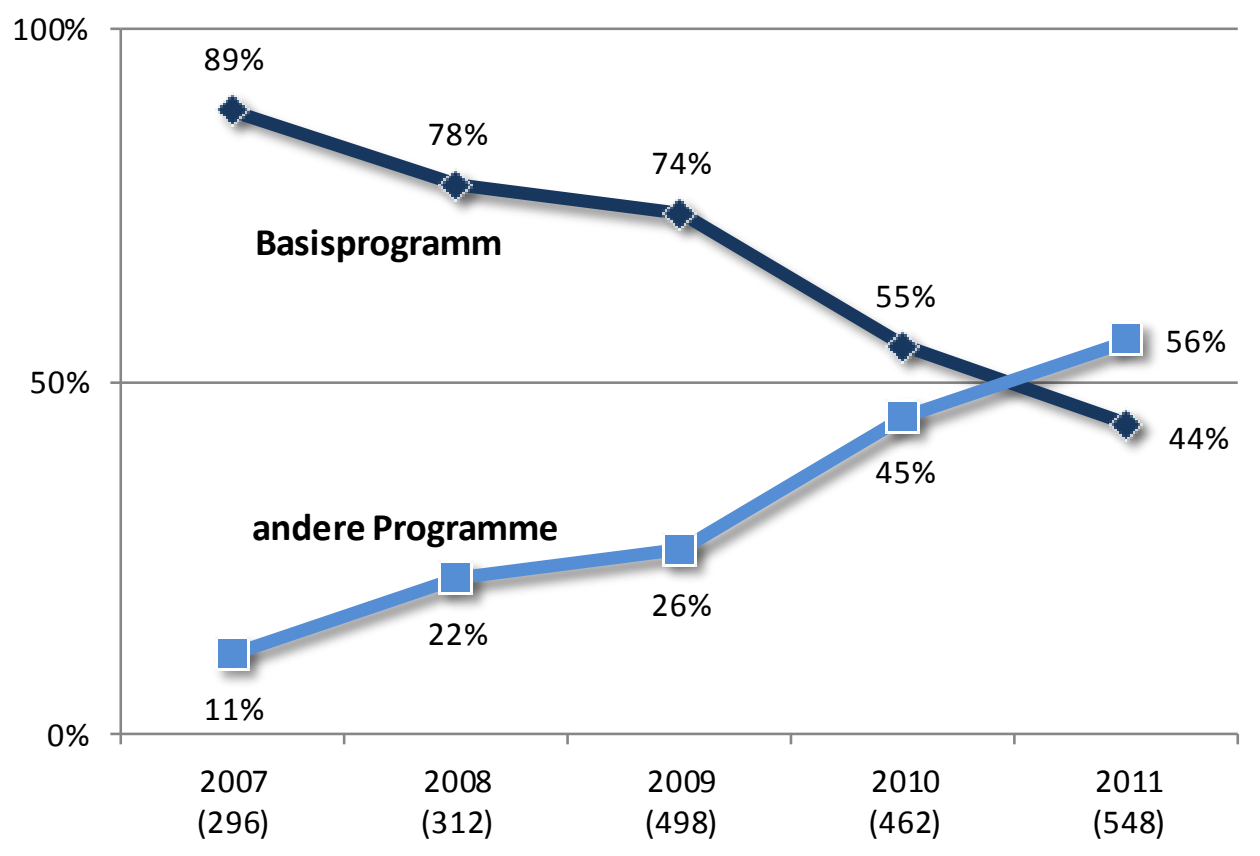

Quelle: KMU Forschung Austria; Jahr des Projektendes; die Zahl der jeweils erfassten Fragebögen sind in Klammer angeführt

Die höheren Zahlen in den einzelnen Programmen erlauben zunehmend spezifischere Aussagen. Deshalb erfolgt dieses Jahr erstmalig eine Auswertung nach den FFG Förderinstrumenten, welche die zugrundeliegenden Interventionslogiken der Projekte repräsentieren. Teilweise werden Instrumente nur in einzelnen Programmen genutzt, aber öfters können Förderinstrumenten Projekte von mehreren Programmen zugeordnet werden. So können Einzelprojekte (C3) nahezu ausschließlich dem Basisprogramm zugeordnet werden, während sich Kooperationsprojekte (C4) aus einer Vielzahl von Projekten aus unterschiedlichen Programmen der FFG Bereiche TP und ALR zusammensetzen (Tabelle 2). 
Tabelle 2 Zuordnung der Programme und erfassten Unternehmensfragebögen zu FFG Förderungs- und Finanzierungsinstrumenten

\begin{tabular}{|c|c|c|c|c|}
\hline Gruppe & Instrument & Programm & $\begin{array}{l}\text { An- } \\
\text { zahl }\end{array}$ & $\begin{array}{l}\text { An- } \\
\text { teil }\end{array}$ \\
\hline Einstieg & C2 Sondierung & $\begin{array}{l}\text { ASAP, Energie der Zukunft, FIT-IT, } \\
\text { Neue Energien 2020, TAKE OFF }\end{array}$ & 23 & $4 \%$ \\
\hline \multirow{4}{*}{$\begin{array}{l}\text { FEI- } \\
\text { Projekt }\end{array}$} & C3 Einzelprojekt & $\begin{array}{l}\text { Basisprogramm, EUROSTARS, } \\
\text { Energie der Zukunft }\end{array}$ & 247 & $45 \%$ \\
\hline & C4 Kooperationsprojekt & $\begin{array}{l}\text { ASAP, benefit, Energie der Zu- } \\
\text { kunft, FIT-IT, IV2S, IV2Splus, } \\
\text { KIRAS, NANO, Neue Energien } \\
\text { 2020, TAKE OFF }\end{array}$ & 201 & $37 \%$ \\
\hline & C5 Leitprojekt & $\begin{array}{l}\text { Energie der Zukunft, IV2S, Neue } \\
\text { Energien } 2020\end{array}$ & 8 & $1 \%$ \\
\hline & C6 Wissenschaftstransfer & BRIDGE & 25 & $5 \%$ \\
\hline Markteinf & C7 Markteinführung IT & AT:net & 7 & $1 \%$ \\
\hline Struktur & C10 Innovationsnetzwerk & COIN & 37 & $7 \%$ \\
\hline \multicolumn{3}{|l|}{ Gesamt } & 548 & $100 \%$ \\
\hline
\end{tabular}

Quelle: FFG und KMU Forschung Austria

\section{Einzelprojekte und Kooperationsprojekte vereinen rd. $80 \%$ der erfassten Pro- jektteilnahmen auf sich}

Bei den Einzelprojekten (C3) der experimentellen Entwicklung und industriellen Forschung dominiert das Basisprogramm mit 242 von 247 Projektteilnahmen.

Abseits der dominierenden Instrumentengruppe FEI-Projekt bilden die Gruppen Einstieg (4\%), Markteinführung (1\%) und Struktur (7\%) rd. ein Achtel der untersuchten Projektteilnahmen ab.

\section{Kleinst- und Kleinunternehmen überdurchschnittlich im TP und SP engagiert, Großunternehmen im Bereich BP}

Mit $48 \%$ wurde fast die Hälfte der geförderten Projekte von Kleinst- und Kleinunternehmen (KU) durchgeführt. ${ }^{1}$ Weitere $38 \%$ der Projektteilnahmen wurden von Großunternehmen abgewickelt, während die mittleren Unternehmen mit $14 \%$ am seltensten partizipierten. KU sind in den Programmbereichen TP und SP überdurchschnittlich engagiert (C2 61\%, C4 54\%, C10 54\%). Bei Projekten des Basisprogramms waren hingegen ,nur $40 \%$ der Teilnehmer KU. Nicht nach Projektbeteiligungen, sondern nach Unternehmen abgegrenzt, waren 51\% der geförderten Unternehmen $\mathrm{KU}, 15 \% \mathrm{MU}$ und $34 \% \mathrm{GU}$. Letztere engagierten sich überwiegend im Basisprogramm sowie im Programm Neue Energien 2020.

1 Die Zuordnung der befragten Unternehmen nach EU-Größenklassen wurde von der FFG vorgenommen. Siehe Anhang für die Definition. 
$38 \%$ der Projekte wurden von Unternehmen mit 1 bis 20 Beschäftigten durchgeführt

Wie auch in den Vorjahren stellen die Unternehmen mit 1 bis 20 Mitarbeiterlnnen (38\%) den größten Anteil im Wirkungsmonitoring. Insbesondere die Instrumente Markteinführung (86\%) und Sondierung (rd. 70\%) werden von dieser Größenklasse bedient. Den größten Anteil an Projektteilnahmen von Unternehmen über $1.000 \mathrm{Be}$ schäftigten weisen mit rd. einem Viertel die BRIDGE Projekte auf.

Tabelle 3 Verteilung der 2011 abgeschlossenen Projekte nach Beschäftigtengrößenklassen der Unternehmen, Anzahl

\begin{tabular}{|l|r|r|r|r|}
\hline $\begin{array}{l}\text { Beschäftigten- } \\
\text { größenklassen }\end{array}$ & Gesamt & EP & KP & $\begin{array}{l}\text { Andere In- } \\
\text { strumente }\end{array}$ \\
\hline $1-20$ & $202(38 \%)$ & $66(28 \%)$ & $88(45 \%)$ & $48(48 \%)$ \\
\hline $21-50$ & $75(14 \%)$ & $40(17 \%)$ & $20(10 \%)$ & $15(15 \%)$ \\
\hline $51-100$ & $44(8 \%)$ & $28(12 \%)$ & $14(7 \%)$ & $2(2 \%)$ \\
\hline $101-250$ & $68(13 \%)$ & $37(16 \%)$ & $21(11 \%)$ & $10(10 \%)$ \\
\hline $251-500$ & $37(7 \%)$ & $21(9 \%)$ & $7(4 \%)$ & $9(9 \%)$ \\
\hline $501-1.000$ & $39(7 \%)$ & $20(8 \%)$ & $16(8 \%)$ & $3(3 \%)$ \\
\hline über 1.000 & $67(13 \%)$ & $26(11 \%)$ & $29(15 \%)$ & $12(12 \%)$ \\
\hline \hline Gesamt & $532(100 \%)$ & $238(100 \%)$ & $195(100 \%)$ & $99(100 \%)$ \\
\hline
\end{tabular}

Quelle: KMU Forschung Austria; EP = Einzelprojekt; KP = Kooperationsprojekt

\section{Weiterhin steigende Anzahl von Projektteilnahmen aus dem Dienstleistungs- bereich}

Forschungsvorhaben wurden zwar wie in der Vergangenheit zum größten Teil im ÖNACE Abschnitt Herstellung von Waren durchgeführt, allerdings ist dieser Wert weiter gesunken und liegt nun bei rd. 37\% (Vorjahr 45\%). Die Branchen Maschinenbau (8\%) sowie die Herstellung von EDV und optischen Geräten (7\%) sind wiederum die am häufigsten geförderten Kategorien im Bereich Herstellung von Waren. Einen deutlichen Zuwachs konnte der ÖNACE Abschnitt wissenschaftlichen und technischen Dienstleistungen verzeichnen, die nun 25\% der eingegangen Fragebögen umfasst (Vorjahr 19\%). Im Bereich Information und Kommunikation verharrt die deutliche Ausweitung des Vorjahres auf einem Niveau von rd. 16\%. Diese Branchenverteilung spiegelt die in Tabelle 4 dargestellte Schwerpunktverlagerung des Wirkungsmonitorings hin zu den insb. Thematischen Programmen wider. 
Tabelle 4 Verteilung der analysierten und 2011 abgeschlossenen Projekte nach ÖNACE-Klassifikation der Projektteilnahmen

\begin{tabular}{|r|c|c|}
\hline \multicolumn{1}{|c|}{ Abschnitt } & Anzahl & Anteil in \% \\
\hline C: Herstellung von Waren & $(203)$ & $(37 \%)$ \\
\hline EDV u. optische Geräte & 37 & $7 \%$ \\
\hline Maschinenbau & 43 & $8 \%$ \\
\hline Chemische Erzeugnisse, Gummi- und Kunststoffwaren & 18 & $6 \%$ \\
\hline Elektrische Ausrüstungen & 25 & $5 \%$ \\
\hline $\begin{array}{r}\text { D: } \text { Snenstige Waren } \\
\text { gung/Beseitigung von Umweltverschmutzung; F: Bau }\end{array}$ & 46 & $8 \%$ \\
\hline $\begin{array}{l}\text { G: Handel; Instandhaltung und Reparatur von KFZ; } \\
\text { H: Verkehr und Lagerei }\end{array}$ & 23 & $4 \%$ \\
\hline J: Information und Kommunikation & 48 & $9 \%$ \\
\hline M: Wissenschaftliche und technische Dienstleistungen & $(139)$ & $(26 \%)$ \\
\hline $\begin{array}{r}\text { Architekten und Ingenieurbüros; } \\
\text { techn., physik., chem. Untersuchung }\end{array}$ & 80 & $15 \%$ \\
\hline Forschung und Entwicklung & 28 & $5 \%$ \\
\hline Sonstige Dienstleistungen & 31 & $6 \%$ \\
\hline Sonstige Branchen bzw. nicht zuordenbar & 48 & $9 \%$ \\
\hline \hline Gesamt & 548 & $100 \%$ \\
\hline
\end{tabular}

Quelle: KMU Forschung Austria; gerundete Werte

\section{Unternehmen mit hoher FuE-Personalintensität enthalten}

Die 459 befragten Unternehmen (mit 548 Projektbeteiligungen) beschäftigen rd. 168.600 Mitarbeiterlnnen, davon sind 18.858 FuE-MitarbeiterInnen (14\% Frauen). 5.156 davon arbeiteten an den geförderten Projekten.

Im Median waren vier FuE-MitarbeiterInnen an einem Projekt beteiligt (Mittelwert: acht).

Die FuE-Personalintensität (Anteil der FuE-Mitarbeiterlnnen an Gesamtbeschäftigten) ist bei KU erwartungsgemäß am höchsten (Median: 42\%), gegenüber 12\% bei MU und $7 \%$ bei GU.

\section{Anteil der Projektleiterinnen gestiegen}

Von den im Jahr 2011 abgeschlossenen Projekten wurden rd. 11\% (59) unter weiblicher Projektleitung durchgeführt. Im Basisprogramm (C3 Einzelprojekt) ist der Anteil mit 9\% unterdurchschnittlich, bei Kooperationsprojekten (C4) mit rd. $12 \%$ und bei den übrigen Instrumenten mit 15\% überdurchschnittlich. Gegenüber den letzten beiden Jahren entspricht dies sowohl in absoluten als auch in relativen Werten einer Zunahme (2009: 4\%; 2010: 9\%). 


\section{Positionierung der Projekte im Portfolio der Unternehmen}

Mit der Frage nach der Positionierung der untersuchten Projekte im Portfolio der jeweiligen Unternehmen lassen sich auf Basis der strategischen Einbettung der Projekte in die Unternehmen etwaige Rückschlüsse auf die Stoßrichtung der Projekte sowie deren ursprüngliche Relevanz für die geförderten Unternehmen ziehen.

Bestehende vs. neue Aktivitätsfelder: Verteilung der Projekte mit bemerkenswerter Konstanz über die Jahre

Für die befragten Unternehmen stellte die Durchführung des geförderten Projektes mit $62 \%$ hauptsächlich ein Baustein in bereits etablierten Aktivitätsfeldern dar, während $35 \%$ der Forschungsprojekte einen Anstoß für ein neues Aktivitätsfeld im Unternehmen. Dies spiegelt die Verteilung der letzten Jahre wieder. Hier scheint es nur in der längeren Frist u.a. aufgrund von Portfolioänderungen in der FFG zu Verschiebungen zu kommen.

Abbildung 2 Verteilung der 2011 abgeschlossenen Projekte nach der Rolle des Projekts im Portfolio des Unternehmens

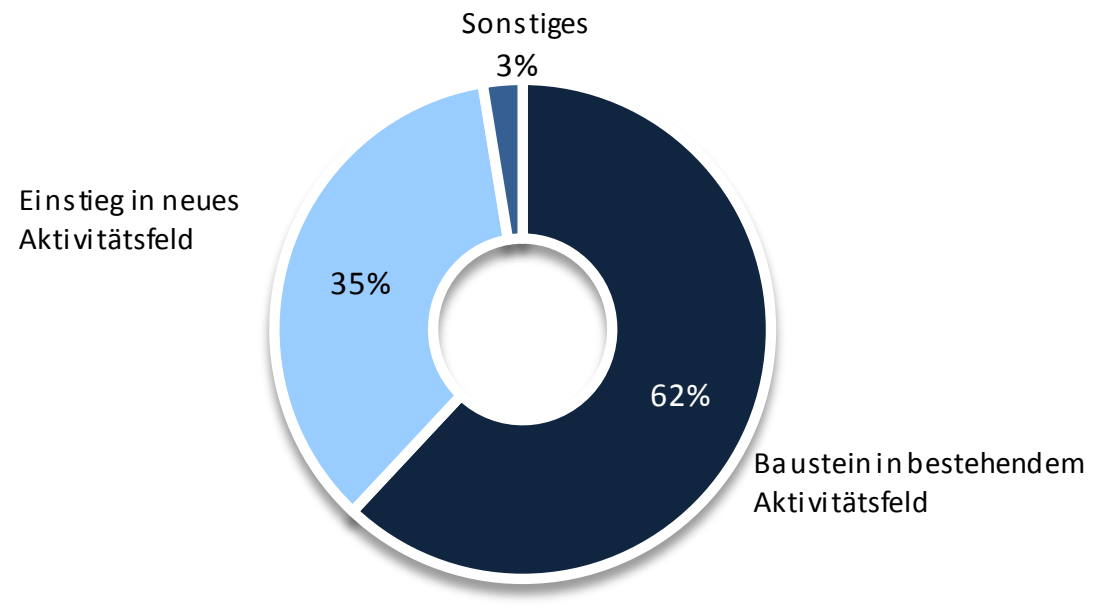

Quelle: KMU Forschung Austria; N=544

Zwischen den einzelnen Instrumenten lassen sich jedoch tlw. deutliche Unterschiede feststellen. Am häufigsten wurden die BRIDGE Projekte (C6: 67\%), und Markteinführungsprojekte (C7: 71\%) als Bausteine bestehender Aktivitätsfelder angesehen. Im 
Gegensatz dazu haben Innovationsnetzwerke (C10) vergleichsweise häufiger ein neues Aktivitätsfeld angestoßen (50\%), während die FEI Projekte (C3-EP 65\%, C4KP 60\%) und Sondierungsprojekte (C2 65\%) dazwischen liegen.

Eine Auswertung nach Unternehmensgrößenklassen zeigt ebenfalls deutliche Unterschiede. Während $68 \%$ der Projektteilnahmen von GU in einem bestehenden Aktivitätsfeld durchgeführt wurden, waren es bei den MU nur 51\% (KU 60\%). Damit waren insbesondere mittelgroße Unternehmen eher an neuen Aktivitätsfeldern interessiert.

\section{Projektteilnahmen dienten primär der Erhaltung bzw. dem Ausbau bestehen- der FuE-Aktivitäten}

Auch die Frage nach dem Anschluss an vorherige FuE-Tätigkeiten vs. dem Einstieg des Unternehmens in FuE zeigt über die letzten drei Jahre ein bemerkenswert stabiles Bild. In immerhin $10 \%$ der untersuchten Fälle stellte das geförderte Forschungsvorhaben die erste FuE Aktivität im Unternehmen (bis zum Zeitpunkt der Projektdurchführung) dar bzw. für $3 \%$ auch die bisher einzige. D.h. im Umkehrschluss, dass 7\%-Punkte bzw. 37 Projektteilnahmen nach Projektabschluss zu weiteren FuETätigkeiten geführt haben. D.h. für $69 \%$ der FuE-Neulinge (37 von 54 Projektteilnahmen) waren die Projekte der Eintritt in weitere FuE.

Tabelle 5 Verteilung der 2011 abgeschlossenen Projekte nach der Rolle des Projekts hinsichtlich der FuE Aktivitäten im Unternehmen

\begin{tabular}{|r|c|c|c|c|}
\hline & Anzahl & Anteil & $\begin{array}{c}\text { EP } \\
\text { bzw. } \\
\text { KP }\end{array}$ & $\begin{array}{c}\text { Andere } \\
\text { Instru- } \\
\text { mente }\end{array}$ \\
\hline $\begin{array}{r}\text { Erste FuE Aktivität im Unternehmen (bis zum Zeit- } \\
\text { punkt der Projektdurchführung) }\end{array}$ & 54 & $10 \%$ & $10 \%$ & $14 \%$ \\
\hline Einzige FuE Aktivität im Unternehmen (bis heute) & 17 & $3 \%$ & $2 \%$ & $7 \%$ \\
\hline Ausbau von bestehenden Aktivitäten & 464 & $87 \%$ & $\begin{array}{l}88 \mathrm{bzw} . \\
89 \%\end{array}$ & $79 \%$ \\
\hline \hline Gesamt & 535 & $100 \%$ & $100 \%$ & $100 \%$ \\
\hline
\end{tabular}

Quelle: KMU Forschung Austria

Hauptverantwortlich für den Ausbau bestehender FuE Aktivitäten zeigen sich die Einzel- (88\%) und Kooperationsprojekte (89\%). In den verbleibenden Instrumentenkategorien gab hingegen ein höherer Anteil an, die Projekte für den Einstieg in FuE Tätigkeiten genutzt zu haben (z.B. Inno-Netz/COIN: 11\%).

Während MU der obigen Verteilung entsprechen, investierten GU zu 94\% in bestehende Aktivitäten; Kleinst- und Kleinunternehmen hingegen in 81\% der Fälle (erste FuE-Aktivität: 15\%). 
Technische Erfolgsrate gesamt auf dem Niveau der Vorjahre; zeigt aber instrumentenspezifische Unterschiede

Einen Projekterfolg aus technischer Sicht konnten 91\% der Projekte erzielen. Diese hohe Erfolgsrate entspricht dem Ergebnis der Vorjahre (89-92\%) und ist dahingehend zu interpretieren, dass die Unternehmen die Projektresultate in einer nutzbringenden Form einsetzen konnten. Dies bedeutet nicht notwendigerweise, dass die bzw. alle ursprünglich gesetzten Ziele erreicht werden konnten.

Der Verlauf der Linie zur technischen Projektzielerreichung spiegelt die mit den Instrumenten verbundene Komplexität sowie Anwendungsnähe der durchgeführten Projekte wider. Speziell die erfassten (nur 8) Leitprojekte konnten aufgrund ihrer Komplexität und damit verbundenen verschiedenen Arten von Risiken nur geringere Erfolgsraten verbuchen.

Abbildung 3 Erreichung der Projektziele aus technischer und wirtschaftlicher Sicht, 2011 abgeschlossene Projekte

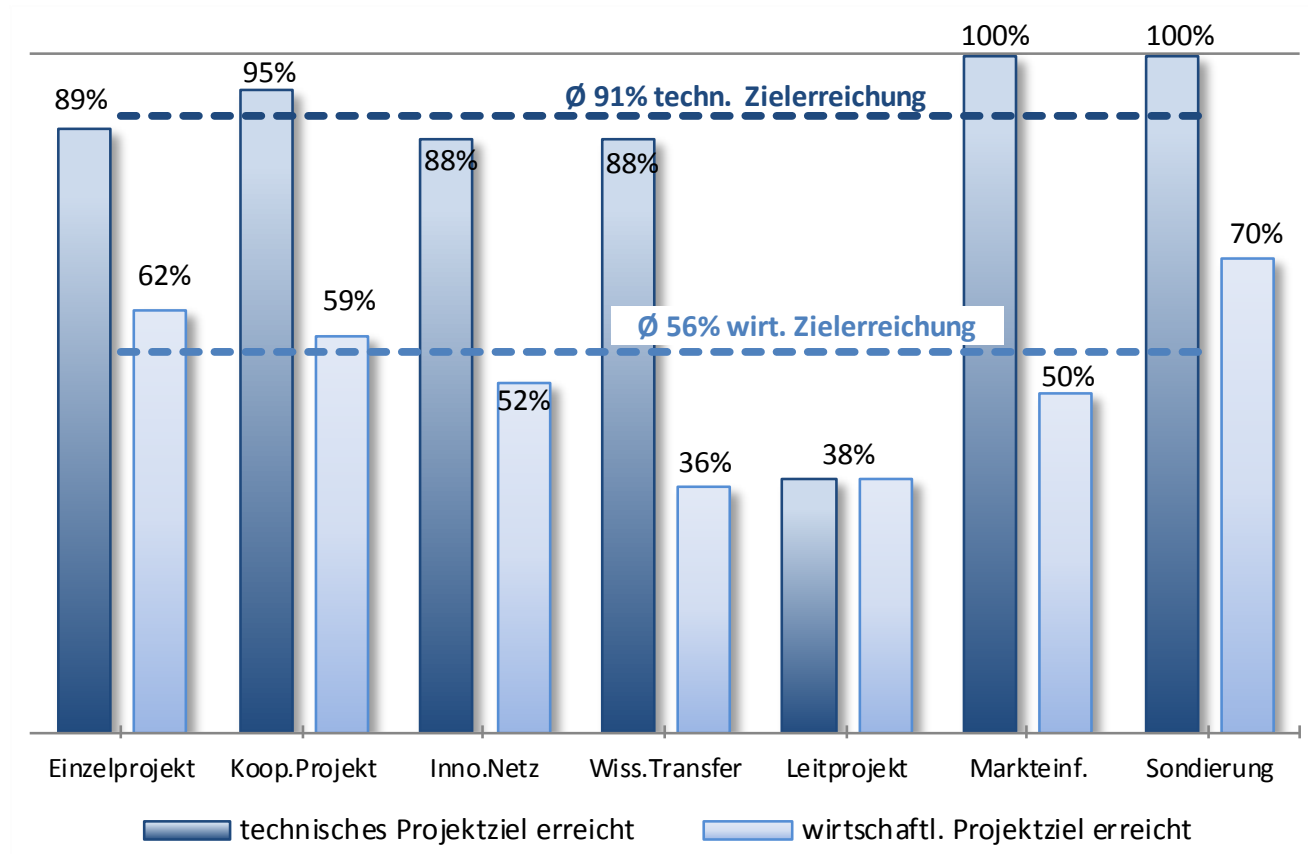

Quelle: KMU Forschung Austria, N=528; sortiert nach der Anzahl von erfassten Projektbeteiligungen

Die Realisierung der wirtschaftlichen Projektziele ist, vier Jahre nach Projektende, noch stark an der Anwendungsnähe der damaligen Projekte gekoppelt.

Die Erreichung einer technischen Lösung führt nicht notwendigerweise auch zu einem Erreichen der wirtschaftlichen Projektziele. Insgesamt konnten in $56 \%$ der Forschungsvorhaben bislang auch die wirtschaftlichen Ziele umgesetzt werden. Damit liegt der Wert am unteren Ende der Vorjahresergebnisse (57-62\%), dies ist jedoch auf die Veränderung der Grundgesamtheit zurückzuführen: Mit den in den letzten 
Jahren zunehmenden Projekten aus dem Bereich TP nimmt der Anteil von Projekten ohne ausdrücklich wirtschaftliche Verwertungsziele zu. Dieses Jahr wurde erstmals separat erfasst, ob ein Projekt überhaupt eine wirtschaftliche Verwertung als Ziel hatte; bei $6 \%$ der Projektteilnahmen war dies nicht der Fall. Ohne die Bereinigung der Resultate erhielte man eine wirtschaftliche Erfolgsquote von rd. 59\%.

Die Einzelprojekte (hauptsächlich Basisprogramm) liegen mit rd. 62\% im guten langjährigen Durchschnitt, die Kooperationsprojekte liegen als zweites wesentliches Instrument nur knapp darunter. Die weiteren Instrumente zeigen ein heterogeneres Bild, wobei durchscheint, dass damit in den verschiedenen Instrumenten auch unterschiedlich hohe wirtschaftliche Erwartungen verbunden sein dürften. Während die Sondierungsprojekte mit 70\% die höchste Zufriedenheit bei der wirtschaftlichen Zielerreichung aufweisen, liegt dieser Wert bei den komplexen Leitprojekten und den grundlagenforschungsnäheren BRIDGE Projekten nur halb so hoch.

\section{Die Barrieren hin zur wirtschaftlichen Zielerreichung sind nach Instrumenten unterschiedlich}

Die 204 Projektteilnehmer mit (derzeit noch) wirtschaftlicher Zielverfehlung konnten auch die dafür wahrgenommenen Gründe angeben.

\section{Abbildung 4 Gründe für wirtschaftliche Zielverfehlung, Mehrfachnennungen}

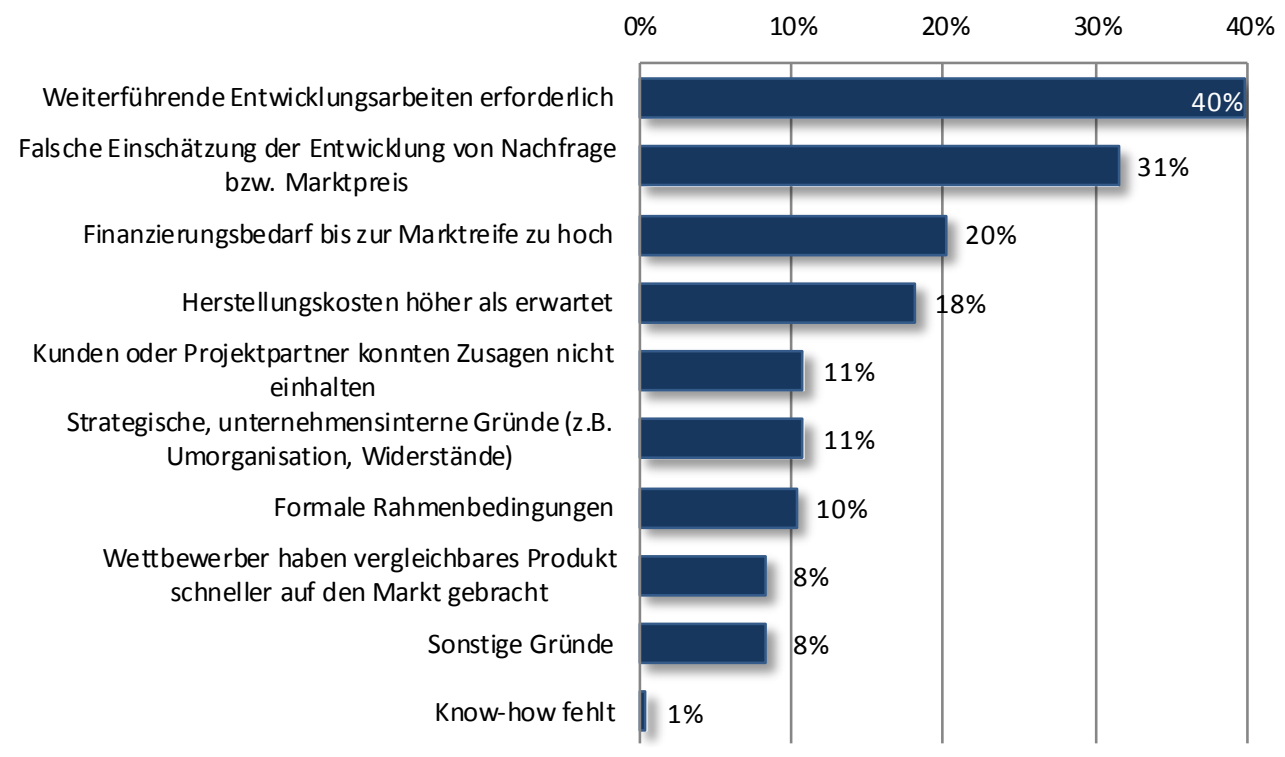

Quelle: KMU Forschung Austria; N=204 
In rd. 40\% dieser Projekte sind noch weiterführende techn. Entwicklungen erforderlich. Dies kann z.B. allein an der Länge des Innovationszyklus liegen, bzw. wurde die Weiterentwicklung mitunter auch aufgrund der nun folgenden Argumente bisher noch nicht umgesetzt. Die nächstwichtigsten Begründungen sind markt- und kostenbedingte Beschränkungen. Während insbesondere markt-, aber auch kostenbedingte Argumente bei Einzel- und Kooperationsprojekten überdurchschnittlich angegeben werden, sind dies bei den weiteren Instrumenten stärker die noch erforderlichen techn. Entwicklungsarbeiten sowie die Sorge um den Finanzierungsbedarf bis zur Marktreife. In Projekten aus den Innovationsnetzwerken, Wissenschaftstransfer sowie Leitprojekte dürften somit aufgrund von längeren Innovationszyklen noch verstärkt zukünftige Verwertungspotenziale liegen, was durchaus zu ihrer Interventionslogik passt.

Großunternehmen zeigen heuer, dass sie insbesondere bei der wirtschaftlichen Verwertung der Projektergebnisse erfolgreicher sind. Dies war jedoch in den vergangenen Jahren nicht der Fall, die sich ausgeglichener präsentierten.

Tabelle $6 \quad$ Erreichung des Projektzieles aus technischer und wirtschaftlicher Sicht nach Unternehmensgrößenklassen, 2011 abgeschlossene Projekte

\begin{tabular}{|c|c|c|c|c|c|c|c|}
\hline & \multicolumn{2}{|c|}{ KU } & \multicolumn{2}{|c|}{ MU } & \multicolumn{2}{|c|}{ GU } \\
\hline & & Anzahl & $\%$ & Anzahl & $\%$ & Anzahl & $\%$ \\
\hline \multirow{4}{*}{$\begin{array}{l}\text { technische } \\
\text { Zielerreichung }\end{array}$} & gesamt & 233 & $88 \%$ & 57 & $76 \%$ & 190 & $91 \%$ \\
\hline & EP & 86 & $86 \%$ & 32 & $82 \%$ & 97 & $91 \%$ \\
\hline & $\mathrm{KP}$ & 99 & $92 \%$ & 17 & $77 \%$ & 67 & $94 \%$ \\
\hline & andere & 48 & $87 \%$ & 8 & $57 \%$ & 26 & $81 \%$ \\
\hline \multirow{4}{*}{$\begin{array}{l}\text { wirtschaftliche } \\
\text { Zielerreichung }\end{array}$} & gesamt & 129 & $49 \%$ & 38 & $51 \%$ & 126 & $60 \%$ \\
\hline & EP & 53 & $53 \%$ & 25 & $64 \%$ & 69 & $64 \%$ \\
\hline & $\mathrm{KP}$ & 53 & $49 \%$ & 8 & $36 \%$ & 41 & $58 \%$ \\
\hline & andere & 23 & $42 \%$ & 5 & $36 \%$ & 16 & $50 \%$ \\
\hline
\end{tabular}

Quelle: KMU Forschung Austria, N=548

Bei der wirtschaftlichen Zielerreichung liegen vier Jahre nach Projektende naheliegender weise die Einzelprojekte voran, auch wenn die anderen Instrumente, wie oben beschrieben, aufgrund der längeren Innovationszyklen die relativ besten Aussichten hinsichtlich noch nicht realisierter, aber zukunftsträchtiger Verwertungspotenziale aufweisen.

Kooperationsprojekte weisen interessanterweise eine sehr hohe technische Zielerreichung aus. Dies gilt es in Zukunft weiter zu beobachten. 


\section{Wirtschaftliche Projektergebnisse}

Der Barwert der Förderungen der vorliegenden und im Jahr 2011 abgeschlossenen 548 Projekte beläuft sich auf rd. $€ 78,4$ Mio. die Gesamtkosten der Projekte in den Unternehmen betrugen knapp $€ 228$ Mio. Die Förderquote ${ }^{2}$ bezogen auf den Barwert liegt im Mittel bei 34\% (Median 45\%). Der durchschnittliche Förderbarwert liegt bei etwa $€ 143.100$ bzw. der Median bei $€ 80.600$.

Tabelle 7 Projektkosten nach Förderungs- und Finanzierungsinstrumenten sowie Unternehmensgröße

\begin{tabular}{|l|r|r|r|r|r|c|}
\hline \multirow{2}{*}{$\begin{array}{c}\text { Instrument / } \\
\text { Größenklasse }\end{array}$} & \multicolumn{2}{|c|}{ Gesamtkosten } & \multicolumn{2}{c|}{ Förderbarwert } & \multicolumn{2}{c|}{ Förderquote } \\
\cline { 2 - 7 } & Mittelwert & Median & Mittelwert & Median & Mittelwert & Median \\
\hline C2 Sondierung & 140.700 & 63.900 & 68.600 & 37.400 & $49 \%$ & $65 \%$ \\
\hline C3 Einzelprojekt & 613.300 & 351.100 & 177.900 & 101.200 & $29 \%$ & $31 \%$ \\
\hline C4 Kooperation & 277.200 & 110.400 & 132.600 & 70.700 & $48 \%$ & $65 \%$ \\
\hline C5 Leitprojekt & 416.900 & 286.700 & 142.000 & 114.000 & $34 \%$ & $38 \%$ \\
\hline C6 Wiss.Transf. & 126.200 & 52.300 & 67.700 & 33.900 & $54 \%$ & $60 \%$ \\
\hline C7 Markteinf. & 464.600 & 378.300 & 107.160 & 94.600 & $23 \%$ & $25 \%$ \\
\hline C10 Inno-Netz & 125.400 & 99.300 & 67.700 & 48.700 & $54 \%$ & $60 \%$ \\
\hline \hline KU & 216.600 & 133.900 & 99.200 & 65.100 & $46 \%$ & $60 \%$ \\
\hline MU & 368.000 & 218.689 & 150.900 & 86.400 & $41 \%$ & $42 \%$ \\
\hline GU & 683.400 & 347.800 & 195.300 & 107.000 & $29 \%$ & $31 \%$ \\
\hline
\end{tabular}

Quelle: KMU Forschung Austria; N=548

Insgesamt wird rd. die Hälfte der Projekte bereits wirtschaftlich verwertet, von weiteren $17 \%$ wird dies noch erwartet.

Von den restlichen Projektteilnahmen war der Großteil immerhin im Sinne der Erkenntniserweiterung für die Unternehmen nützlich; dies umfasst das Wissen was nicht funktioniert, anders funktionieren könnte, bzw. am Markt platziert werden könnte.

6\% der Projektteilnahmen (30) lassen sich FuE-Teilprojekten zuweisen, bei denen das beteiligte Unternehmen kein unmittelbares wirtschaftliches Verwertungsziel verfolgte. Vielmehr standen in diesen Projekten die Sondierung von Wissen, der Brückenschlag zwischen Grundlagen- und angewandter Forschung, der Zugang zu Infrastrukturen und Technologien oder gesellschaftsrelevante Fragestellungen im Vordergrund.

2 Anteil Barwert der Förderung an den Gesamtkosten des Projekts 
Tabelle 8 Wirtschaftliche Verwertung der vor 4 Jahren abgeschlossenen Projekte

\begin{tabular}{|l|r|r|r|r|}
\hline \multirow{2}{*}{ Die Projektergebnisse ... } & \multicolumn{2}{|c|}{ Projektbeteilig. } & \multicolumn{2}{c|}{ Förderbarwert } \\
\cline { 2 - 5 } & Anzahl & \multicolumn{1}{|c|}{$\%$} & Mio. $€$ & $\%$ \\
\hline $\begin{array}{l}\text { werden im Unternehmen bereits wirtschaftlich } \\
\text { verwertet }\end{array}$ & 266 & $49 \%$ & 45,9 & $60 \%$ \\
\hline $\begin{array}{l}\text { werden in Zukunft im Unternehmen wirtschaftl. } \\
\text { verwertet werden können }\end{array}$ & 95 & $17 \%$ & 14,3 & $18 \%$ \\
\hline $\begin{array}{l}\text { sind im Unternehmen nicht wirtschaftl. verwertbar, } \\
\text { aber sinnvoll für Erkenntniserweiterung }\end{array}$ & 132 & $24 \%$ & 13,6 & $17 \%$ \\
\hline können im Unternehmen nicht verwertet werden & 23 & $4 \%$ & 2,6 & $3 \%$ \\
\hline $\begin{array}{l}\text { Ein unmittelbares wirtschaftliches Verwertungsziel } \\
\text { stand nicht im Vordergrund }\end{array}$ & 30 & $6 \%$ & 1,6 & $2 \%$ \\
\hline Keine Angabe & 2 & $1 \%$ & 0,3 & $0 \%$ \\
\hline \hline Gesamt & 548 & $100 \%$ & 78,4 & $100 \%$ \\
\hline
\end{tabular}

Quelle: KMU Forschung Austria, N=548

Ein überproportional hoher Teil des Förderbarwertes fällt auf Projekte, die bereits wirtschaftlich verwertet werden.

\section{Abbildung 5 Wirtschaftliche Verwertung der vor 4 Jahren abgeschlossenen Projekte}

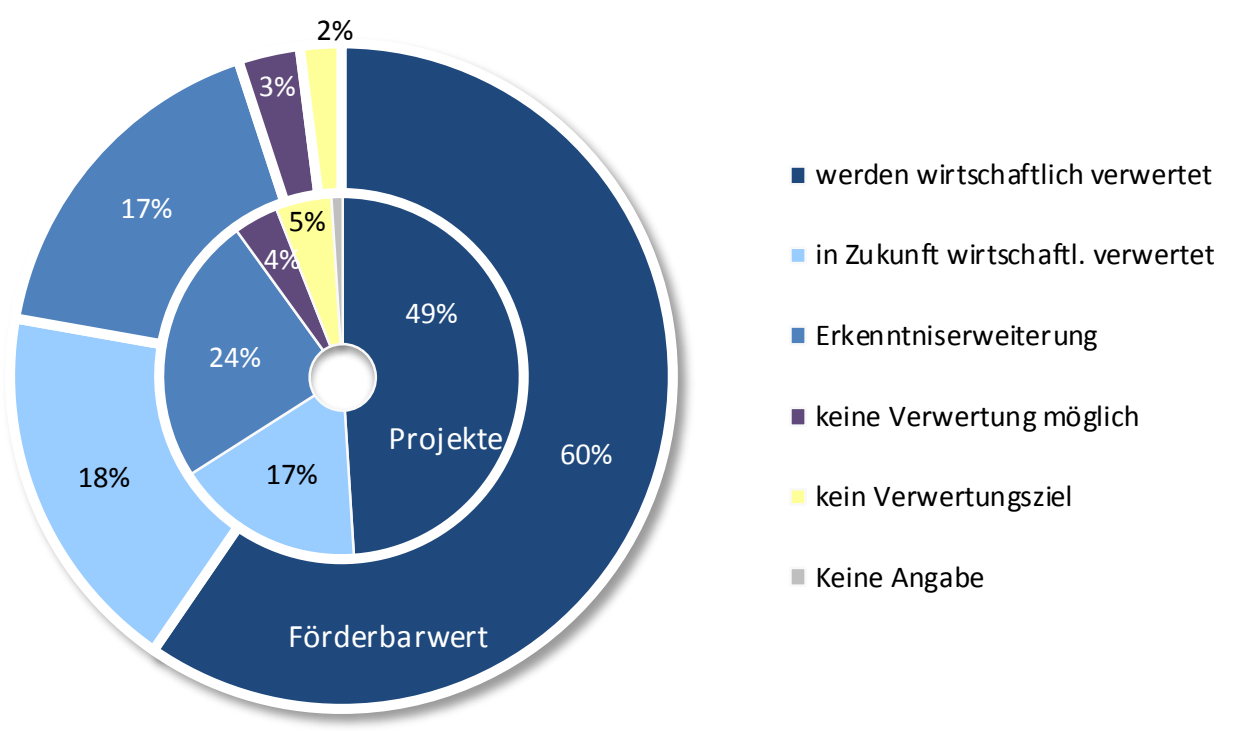

Quelle: KMU Forschung Austria, N=548 Projekteilnahmen mit einem Förderbarwert von 78,4 Mio. $€$

Im Vergleich zu den Ergebnissen der Vergangenheit verzögert sich die Verwertung etwas, d.h. zwischen den Kategorien ,bereits verwertet' und ,in Zukunft verwertbar'. Der Anteil für Letzteres lag traditionell bei $9-10 \%$. 


\section{Einzelprojekte bei wirtschaftlicher Verwertung auf gewohnt hohem Niveau, andere Instrumente benötigen mehr Zeit bzw. bergen höhere Risiken}

Betrachtet man die Verwertungsresultate der einzelnen Instrumenten zeigt sich, dass Einzelprojekte bereits zu rd. zwei Drittel wirtschaftlich verwertet werden (65\%) bzw. steht diese bei $13 \%$ bevor, und können damit nahtlos an die hohen Werte der Vergangenheit anschließen.

Bei Projekten des Wissenschaftstransfers dauert die Verwertung der Projektergebnisse erwartungsgemäß deutlich länger: $24 \%$ an derzeitiger Verwertung stehen weitere $40 \%$ noch erwarteter Kommerzialisierung gegenüber.

Bei Projekten der Instrumente Markteinführung (AT:net), Innovationsnetzwerk (COIN), Wissenschaftstransfer (BRIDGE), Leitprojekt, aber auch Kooperationsprojekt scheinen ebenfalls Risiken unterschiedlicher Art verstärkt aufzutreten. Davon zeugen zumindest rd. 30-40\% an Projektteilnahmen, die zu keiner Kommerzialisierung in den Unternehmen beitragen werden. Aus den qualitativen Rückmeldungen an einer anderen Stelle im Fragebogen wissen wir, dass die wichtigsten Risiken sowohl technologische, Markt- bzw. Nachfrage-, Finanzierungs- wie auch Kooperationsrisiken umfassen (in dieser Reihenfolge).

Beim Instrument Markteinführung (AT:net) ist die Verteilung insofern interpretationsbedürftig, als aufgrund der Marktnähe dieser Projekte eine alleinige Erkenntniserweiterung ein mageres Resultat darstellt. Die aufgrund der kleinen Zahl im Sample nur zwei Projektteilnahmen hatten die Marktnachfrage bzw. den Marktpreis falsch eingeschätzt. Die beiden Leitprojektteilnahmen, die keine Verwertung angaben, lassen sich auf die mangelnde technische Zielerreichung zurückführen. In weiterer Folge wurden die Vorhaben eingestellt bzw. waren die Ergebnisse nach Auskunft der Unternehmen nicht für den geplanten Zweck einsetzbar. 


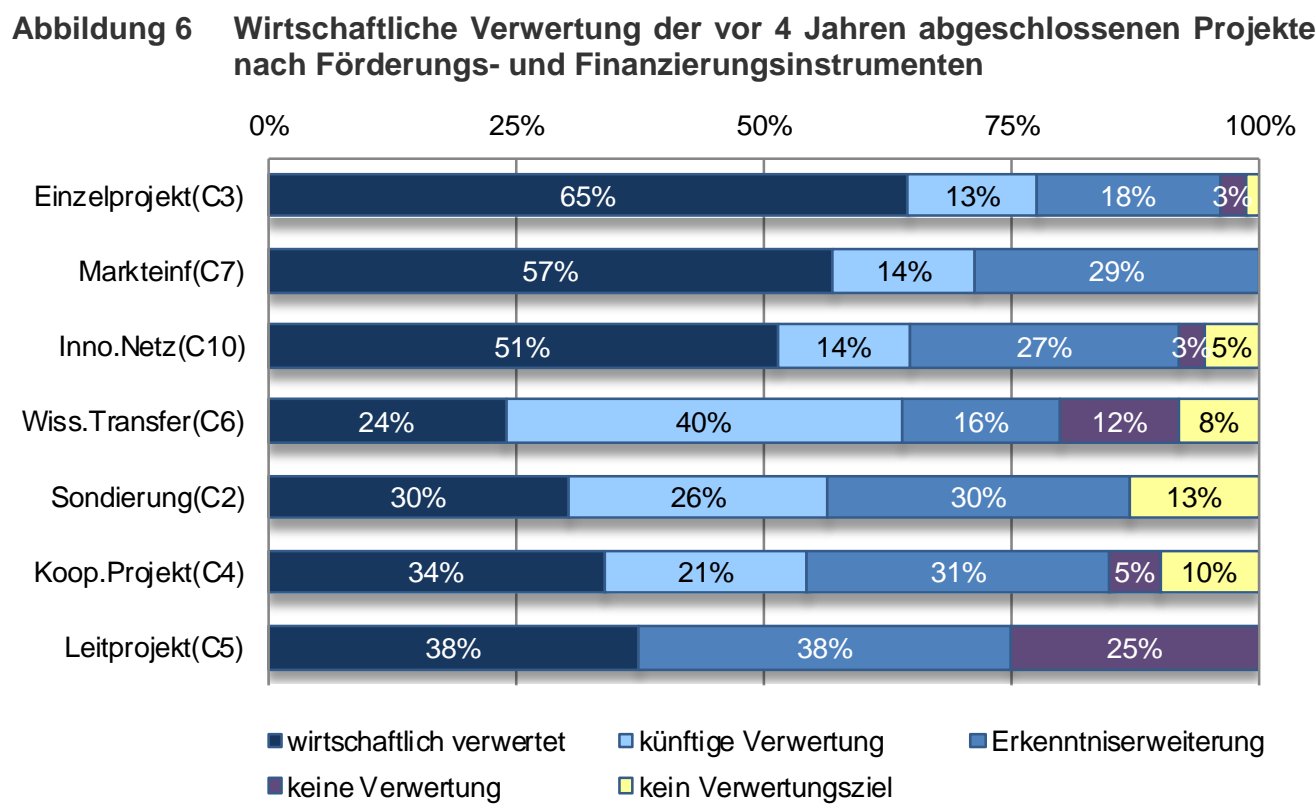

Quelle: KMU Forschung Austria, N=548

Aus der untenstehenden Abbildung lässt sich ableiten, dass zwei Drittel der ausbezahlten bzw. zugesagten Fördermittel in Projekte flossen, deren wirtschaftliche Projektziele bislang erreicht wurden. Nach Anzahl der Projekte liegt dieser Wert bei 56\% (siehe Abbildung 3).

Im Unterschied zu einer identen Auswertung im Jahr 2013 ist das Verhältnis der Förderbudgetverteilung mit/ohne wirtschaftliche Zielerreichung von KU dieses Jahr etwas ungünstiger. Dies könnte auf das sich ändernde Portfolio der FFG zurückzuführen sein (TP), sollte jedoch in den kommenden Jahren noch weiter beobachtet werden. 
Abbildung 7 Fördervolumen der abgeschlossenen Projekte mit bzw. ohne wirtschaftlicher Zielerreichung aus den FFG-geförderten Projekten, nach Größenklassen und Form der Zuwendung

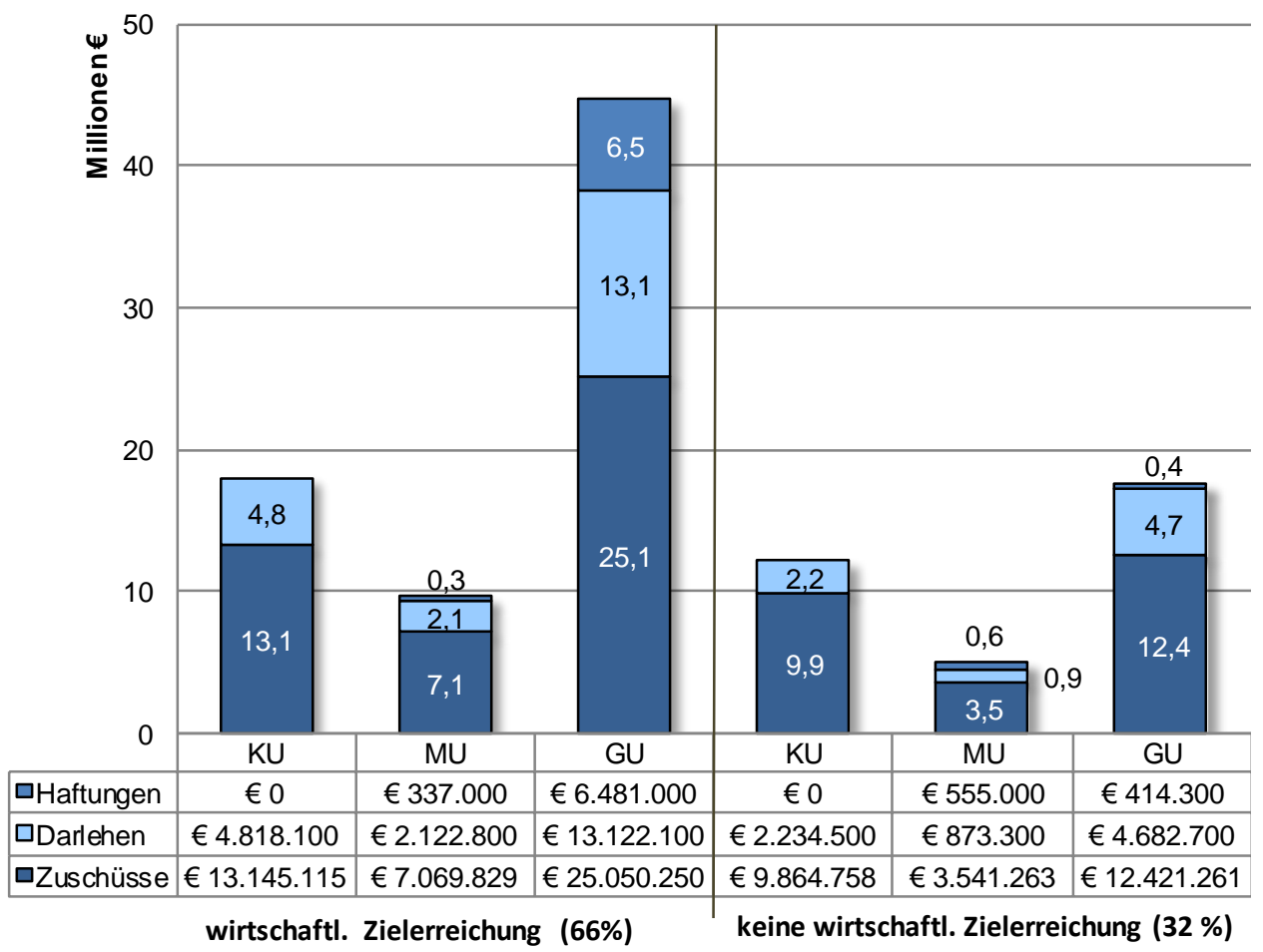

Quelle: KMU Forschung Austria, N=497 (66\% = Fördervolumen bereinigt um Teilprojekte ohne wirtschaftliches Verwertungsziel)

Großunternehmen kommerzialisieren erfolgreicher; tun dies vermehrt aufgrund von (inkrementellen) Innovationen bei bereits eingeführten Produkten und Verfahren.

Wie auch in den vergangenen Jahren stellen wir fest, dass Großunternehmen über Vorteile bei der Verwertung der Forschungsergebnisse verfügen (GU: 55\% der Projektteilnahmen bereits verwertet und $17 \%$ zukünftig vs. KU: $44 \%$ und $18 \%$ ). Im Vergleich zu kleineren Unternehmen investierten GU vermehrt in veränderte Produkte und Verfahren, mit denen man vier Jahre nach Projektende leichter Umsätze erzielen kann, da diese bereits eingeführte Produkte und Prozesse betreffen.

Die Anteile der Auslandsumsätze zeigen sich über die Jahre bemerkenswert stabil

Lizenzerlöse, Zusatzumsätze und Umsatzsicherungen stellen eine Einschätzung der direkten Auswirkungen der geförderten Projekte dar. Hier sind keine Substitutionsoder Multiplikator-Effekte berücksichtigt, wie dies eine Schätzung der gesamtwirtschaftlichen Netto-Effekte erfordern würde. Wie die nachfolgende Tabelle zeigt, wurde der Großteil dieser Lizenzerlöse, Zusatzumsätze und Umsatzsicherungen im 
Ausland erzielt, was die traditionell hohe Exportorientierung der geförderten Unternehmen unterstreicht. Der Schwerpunkt der Effekte liegt dieses Jahr eher bei der Umsatzsicherung (in GU).

Tabelle 9 Direkte wirtschaftliche Ergebnisse der im Jahr 2011 abgeschlossenen Projekte, in Mio. $€$

\begin{tabular}{|r|c|c|}
\hline & $\begin{array}{c}\text { Direktes wirt. Ergebnis } \\
\text { (in Mio. } € \text { ). }\end{array}$ & $\begin{array}{c}\text { davon im Ausland } \\
\text { (in Mio. } € \text { ) }\end{array}$ \\
\hline Verwertung der Ergebnisse durch: & & $19,4(79 \%)$ \\
\hline Lizenzerlöse & 24,7 & $430,2(88 \%)$ \\
\hline Zusatzumsätze & 487,8 & $896,1(81 \%)$ \\
\hline Umsatzsicherung & 1107,8 & \\
\hline
\end{tabular}

Quelle: KMU Forschung Austria, N=164 Unternehmen (Anzahl jener Befragten, die bei Lizenzerlösen, Zusatzumsätzen oder Umsatzsicherung eine eindeutige Summe angeben konnten)

Lizenzerlöse wurden jedoch überwiegend von $\mathrm{KU}$ erwirtschaftet. Insgesamt 27 der Projekte mit Lizenzerlösen sind den Einzelprojekten zuzuordnen. 65 Teilnehmer meldeten, dass bislang keine Lizenzierung der Projektergebnisse erfolgt ist, obwohl sie das Projektergebnis prinzipiell als lizenzierbar ansehen.

Zusätzliche Umsätze konnten im Zuge von insgesamt 163 Projekten erzielt werden, wobei 128 für die Zusammensetzung der obenstehenden Summe verantwortlich zeichnen. Innerhalb der Einzelprojekte konnten 47\% der Teilnehmer zusätzliche Umsätze auf Grund der Projektdurchführung generieren, während es bei den anderen Instrumenten unter 20\% waren.

Einen Beitrag zur Umsatzsicherung identifizierten rd. 27\% der Befragten (146; davon 99 mit Umsatzangaben). Bei den Einzelprojekten trugen 41\% der untersuchten Projektteilnahmen zur Sicherung von Unternehmensumsätzen bei (Kooperationsprojekte: $15 \%$, andere Instrumente: $17 \%)$. 


\section{Fördermultiplikator des Basisprogramms etwas rückläufig}

Der Fördermultiplikator stellt eine simple Input/Output Relation der geförderten Projekte dar. Als Maß für den Input wird der Barwert der vergebenen Fördermittel herangezogen. Das Maß für den Output ist die Summe der durch die jeweiligen Projekte realisierten Lizenzerlöse und Zusatzumsätze. Aufgrund der Einfachheit dieses Indikators ist weniger die absolute Höhe dessen interessant, sondern eher der relative Unterschied zwischen unterschiedlichen Programmen (mit ihren spezifischen Interventionslogiken) sowie die Veränderung des Multiplikators über die Zeit.

\section{Abbildung 8 Fördermultiplikator im Zeitverlauf}

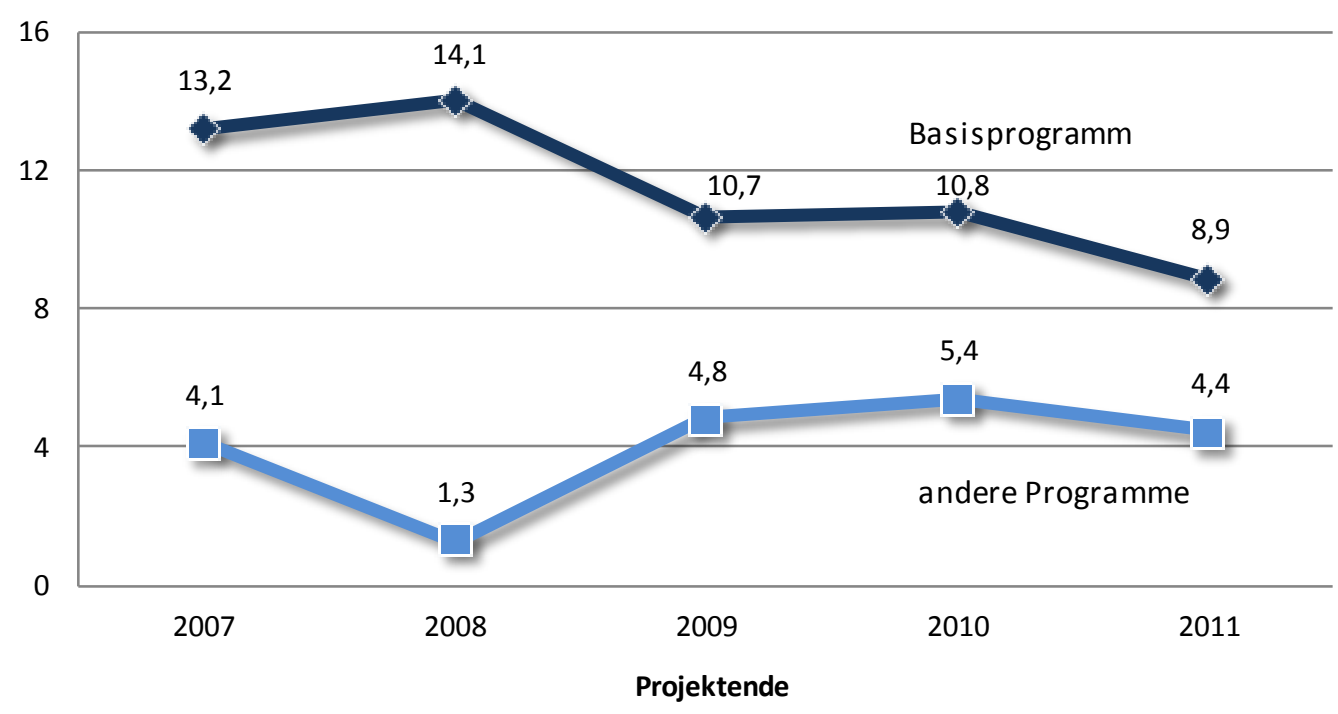

Quelle: KMU Forschung Austria. Eine Auswertung nach Förderinstrumenten würde ein sehr ähnliches Ergebnis liefern. Um eine kohärente Darstellung im Zeitverlauf sicherzustellen, wurde die ursprüngliche Darstellungsart nach Programmen gewählt.

Der Fördermultiplikator des Basisprogramms liegt 2015 bei 8,9 und zeigt damit einen Rückgang. Obwohl die Projekte im selben Umfang wie in den letzten Jahren wirtschaftlich verwertet werden, ist die Summe der Zusatzumsätze geringer. Hier scheinen sich die Unternehmen bei den anwendungsorientierten Projekten Grosso Modo immer noch schwer zu tun, die Vorkrisenwerte zu erreichen.

Der Fördermultiplikator der anderen Programme bewegt sich mit 4,4 in der üblichen Bandbreite (dominiert durch die Kooperationsprojekte mit 4,7). 


\section{Beschäftigungseffekte}

Der Zusammenhang von Innovation und Arbeitsplatzeffekten ist komplex, wie akademische Studien über die Jahre immer wieder zeigen. Während aufgrund von Prozessinnovationen tendenziell eher Kosteneinsparungen und damit verbunden negative Beschäftigungseffekte resultieren können, wird bei Produkt- und Dienstleistungsinnovation tendenziell ein Beschäftigungswachstum oder zumindest deren Sicherung assoziiert, und hat darüber hinaus noch qualitative Effekte auf die Art und Qualität der Arbeitsplätze. Das Wirkungsmonitoring zielt auf die grobe Erfassung der quantitativen Effekte ab.

Die erfassten Projektteilnahmen zeichnen für einen direkten Beschäftigungseffekt von rd. 2.300 Arbeitsplätzen (nach Köpfen) verantwortlich. Diese Summe ist der Saldo der durch die Projektverwertung gesicherten (1.366) und neu geschaffenen (988) Arbeitsplätze abzüglich der freigesetzten MitarbeiterInnen (20). ${ }^{3}$

In den ersten 4 Jahren nach Projektende entfalteten $48 \%$ der Projektbeteiligungen positive Wirkungen auf die Erhaltung und Schaffung von Arbeitsplätzen (2014: 50\%, 2013: 52\%). Dieser leichte Rückgang ist auf den höheren Anteil von Projekten jenseits des Basisprogramms zurückzuführen.

Im Basisprogramm ist dieser Wert über die Jahre sehr konstant (2013-15: 59-60\%). Bislang wurden neue Arbeitsplätze durch 31\% der Projektteilnahmen von MU (nach EU-Definition), $28 \%$ von KU und $26 \%$ von GU geschaffen. Die Sicherung von Arbeitsplätzen erfolgte am häufigsten in GU (28\% aller Projektteilnahmen durch GU), gefolgt von KU (24\%) und MU (23\%). Allein nach Beschäftigtenkategorien sieht die Verteilung folgendermaßen aus.

3 Diese Beschäftigungseffekte sind ausschließlich auf die Verwertung der Projekte zurückzuführen. Dies ist im Unterschied zu den Auswirkungen auf die FuE-Mitarbeiterentwicklung während bzw. als Folge der Projektdurchführung zu sehen, wie weiter hinten beschrieben. 
Abbildung 9 Beschäftigungseffekte der im Jahr 2011 abgeschlossenen Projekte nach Beschäftigtengrößenklassen der Unternehmen, Anzahl nach Köpfen

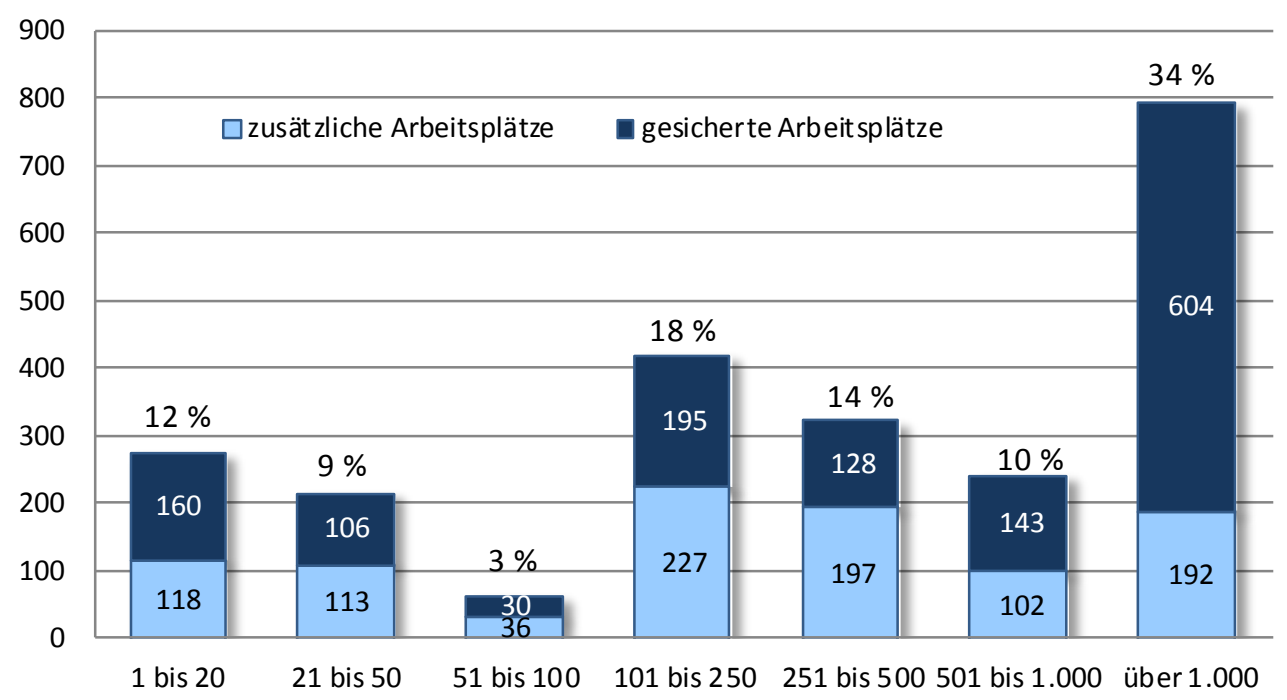

Quelle: KMU Forschung Austria, N=543; Nota bene: dies sind keine VZÄ

Die Schaffung und Sicherung neuer Arbeitsplätze erfolgt am häufigsten im Rahmen von Einzelprojekten. Rd. 40\% dieser Projektteilnahmen führten zu neuen Arbeitsplätzen und $46 \%$ trugen zur Sicherung bei. Der Median der Beschäftigungseffekte liegt bei zwei neuen bzw. fünf gesicherten Beschäftigten. Diese Anteile können in den Kooperationsprojekten (22\% neue bzw. 35\% gesicherte Arbeitsplätze) und den anderen Instrumenten (18\% bzw. 25\%) nicht erreicht werden. Die Mediane betragen jeweils zwei Mitarbeiter.

Interne bzw. externe FuE-Beschäftigungseffekte resultieren bei $57 \%$ aller Projektbeteiligungen; extern rekrutierte FuE-MitarbeiterInnen werden nach Projektende tendenziell noch aufgestockt

Beschäftigungseffekte für FuE-MitarbeiterInnen (FuE-MA) während bzw. nach der Projektlaufzeit lassen sich bei $57 \%$ der 548 Projekte feststellen, wobei diese auf externe und interne Rekrutierung zurückzuführen sind. Durch 215 Projektbeteiligungen (39\% aller Projektteilnahmen) kam es zu externen Rekrutierungen, zusätzlich gaben 29\% der Befragten (161) an, interne Personalneuzuteilungen getroffen zu haben. Während der Projektdurchführung wurden 458 FuE-MitarbeiterInnen extern rekrutiert und weitere 539 nach Abschluss eingestellt. Interne Personalrocharden betrafen 355 Mitarbeiterlnnen während der Projektdurchführung bzw. 123 MitarbeiterInnen nach Abschluss des Projekts. 
Tabelle 10 Externe und interne Rekrutierung von Forschungspersonal

\begin{tabular}{|l|c|c|c|c|}
\hline \multirow{2}{*}{} & \multicolumn{2}{|c|}{ Extern } & \multicolumn{2}{c|}{ Intern } \\
\cline { 2 - 5 } & $\begin{array}{c}\text { während } \\
\text { Projekt. / MA }\end{array}$ & $\begin{array}{c}\text { danach } \\
\text { Projekt. / MA }\end{array}$ & $\begin{array}{c}\text { während } \\
\text { Projekt. / MA }\end{array}$ & $\begin{array}{c}\text { danach } \\
\text { Projekt. / MA }\end{array}$ \\
\hline EP & $37 \%(91) / 318$ & $28 \%(70) / 346$ & $26 \%(65) / 199$ & $12 \%(29) / 51$ \\
\hline KP & $20 \%(40) / 108$ & $18 \%(37) / 172$ & $25 \%(50) / 117$ & $7 \%(14) / 58$ \\
\hline Andere & $19 \%(19) / 32$ & $14 \%(14) / 21$ & $25 \%(25) / 39$ & $12 \%(12) / 14$ \\
\hline Gesamt & $\mathbf{2 7 \% ( 1 5 0 ) / 4 5 8}$ & $\mathbf{2 2 \% ( 1 2 1 ) / 5 3 9}$ & $\mathbf{2 6 \% ( 1 4 0 ) / 3 5 5}$ & $\mathbf{1 0 \% ( 5 5 ) / 1 2 3}$ \\
\hline
\end{tabular}

Quelle: KMU Forschung Austria; N=310 Projektbeteiligungen die zumindest in einer der beiden Kategorien FuE-MA rekrutiert haben; Leseanleitung: 37\% der Einzelprojektbeteiligungen (=91) haben während der Projektumsetzung 318 MA extern rekrutiert.

Die Einzelprojekte zeigen eine weitgehend überdurchschnittliche Affinität für FuEPersonalrekrutierungen, speziell bei der Anwerbung von externen FuE-MA. Dabei zeichnet rd. die Hälfte der Einzelprojektteilnehmer (51\%) für rd. zwei Drittel aller externen Rekrutierungen verantwortlich. Weiter wurde in 31\% der Kooperationsprojekte und $28 \%$ der anderen Instrumente auf Externe zurückgegriffen. Interne Rochaden haben bei 78 Einzelprojekten (32\%), 56 Kooperationsprojekten (28\%) und 27 Projekten (27\%) der anderen Instrumente stattgefunden.

\section{Leicht steigender Fördermitteleinsatz je geschaffenem oder gesichertem Ar- beitsplatz}

Der Barwert der Fördermittel je gesichertem bzw. geschaffenem Arbeitsplatz setzt den Einsatz von Steuermitteln ins Verhältnis zu etwaigen Arbeitsplatzeffekten. In den im Jahr 2011 abgeschlossenen Projekten wurden durchschnittlich rd. € 33.600 an Fördermitteln eingesetzt, um einen Arbeitsplatz zu sichern bzw. zu schaffen. Wie auch im Vorjahr sind die Unterschiede zwischen Basisprogramm und den anderen Programmen tlw. hoch, und lassen sich zum einen mit den unterschiedlichen Interventionslogiken erklären. Über alle Größenklassen hinweg zeigt sich, dass die Barwerte der Einzelprojekte (Basisprogramm) jene der verbliebenen Instrumentenkategorien unterschreiten.

Die Werte je Beschäftigtengrößenklasse sind auf ein Jahr bezogen nicht sehr aussagekräftig. Dieser Effekt kann gemildert werden, indem die Daten von mehreren Jahren in die Berechnung aufgenommen werden. 
Abbildung 10 Durchschnittlicher Barwert der Fördermittel je gesicherten und geschaffenen Arbeitsplatz der in den Jahren 2007 bis 2011 abgeschlossenen Projekte, nach MA-Größenklassen

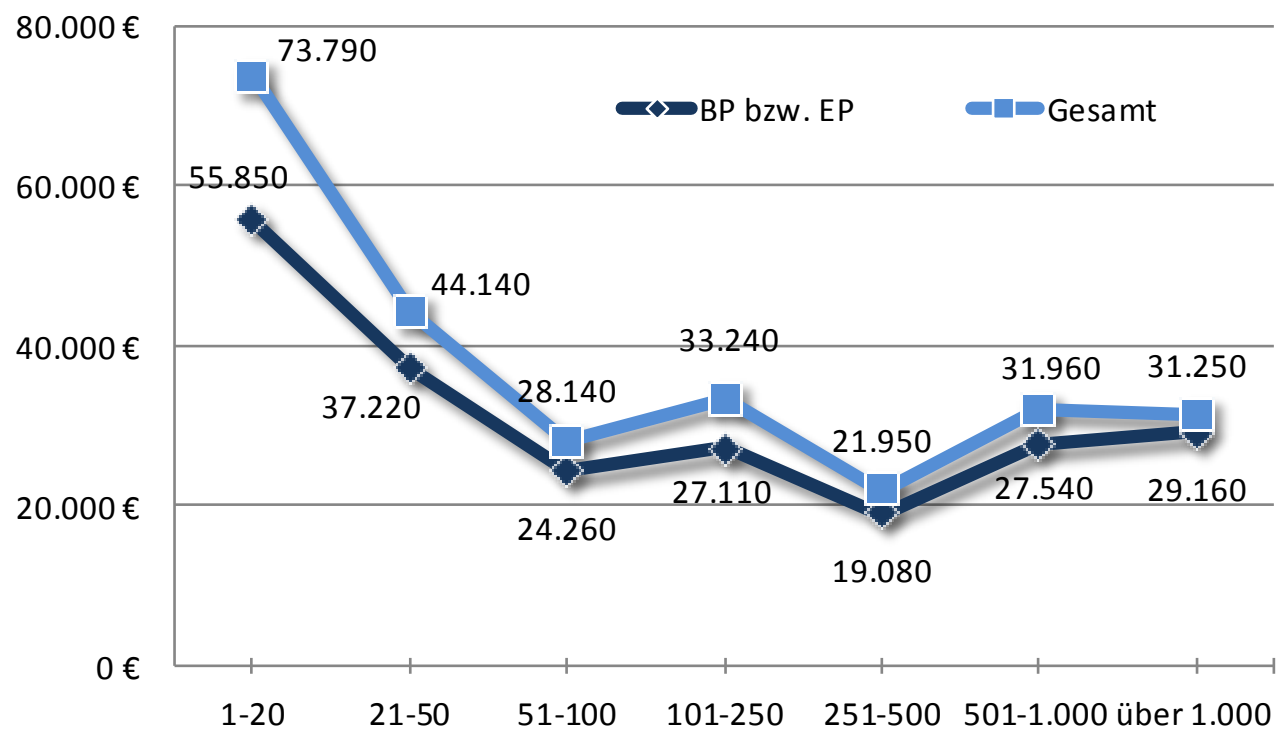

Quelle: FFG und KMU Forschung Austria; gerundet; BP = Basisprogramm, EP = Instrument Einzelprojekt; die Werte der Jahre 2007-2010 beziehen sich auf das Basisprogramm; mit 2011 wurden nun erstmals einige weitere Einzelprojekte aufgenommen, die das Ergebnis aber kaum beeinflussen. Die anderen Instrumente werden nicht gesondert dargestellt, da die anfangs noch geringe Projektzahl größere Schwankungen in den Größenklassen verursacht.

Im Durchschnitt der Jahre 2007 bis 2011 wurden rund $€ 34.590$ an Fördermitteln eingesetzt, um einen zusätzlichen Arbeitsplatz zu schaffen bzw. zu sichern.

Bei kleineren Unternehmen ist tendenziell ein höheres Fördervolumen je geschaffenem oder gesichertem Arbeitsplatz nötig. Dieses Verhältnis sinkt mit zunehmender Beschäftigtenzahl und steigt wieder bei den Großunternehmen an. Das Zielsystem einiger der anderen Programme hat nicht primär Arbeitsplatzbeschaffung als Fokus. Es sei jedoch darauf hingewiesen, dass es sich auch hier lediglich um Näherungswerte handelt, da die projektbezogenen Wirkungen insbesondere bei Großunternehmen Unschärfen aufweisen. Dies drückt sich unter anderem auch in der Zahl jener Unternehmen aus, die Angaben Arbeitsplätze gesichert bzw. geschaffen zu haben, allerdings keine Zahlen nennen konnten. 


\section{Auswirkungen auf die Forschungs-, Entwicklungs-, und Innovationsaktivitäten der Unternehmen}

In 24\% der geförderten Projekte wurden Schutzrechte angemeldet; Diese konzentrieren sich stark auf einzelne Projekte (insb. Einzel- und Leitprojekte), in denen oft Patentfamilien (von Großunternehmen) eingereicht wurden.

Die Schutzrechtanmeldungen aufgrund von 129 Projektbeteiligungen resultieren in rd. 24\% der geförderten Projekte und entsprechen dem Wert des Vorjahres. Nachvollziehbarerweise ist der höchste Anteil bei den Leitprojekten zu finden. Vier von sieben Projektteilnahmen führten zu Schutzrechtsanmeldungen, gefolgt von den Einzelprojekten mit $36 \%$ und den Instrumenten Kooperationsprojekt/ Inno.Netz/ Wiss.Transfer in der Bandbreite von 15-17\%.

Nachvollziehbarerweise melden Unternehmen Schutzrechte primär in Eigenregie an. Diese Vorgehensweise wird meist bereits zu Beginn der Projekte im Rahmen von Konsortialverträgen geregelt. Trotzdem werden ob der intensiven Zusammenarbeit manchmal auch gemeinsame Schutzrechte erworben. Dies passiert naheliegender weise eher bei Kooperationsprojekten und Innovationsnetzwerken.

Tabelle 11 Anmeldung von Schutzrechten

\begin{tabular}{|l|l|c|c|}
\hline \multicolumn{2}{|l|}{ Schutzrechte } & eigenes Unternehmen & mit Projektpartner \\
\hline \multirow{2}{*}{ Technische } & Patente & 393 (97 Teilnahmen) & 19 (14 Teilnahmen) \\
\cline { 2 - 4 } & $\begin{array}{l}\text { Gebrauchsmuster, Sorten- } \\
\text { oder Halbleiterschutz, etc. }\end{array}$ & 22 (19 Teilnahmen) & 1 (zwei Teilnahmen) \\
\hline $\begin{array}{l}\text { Nicht- } \\
\text { technische }\end{array}$ & $\begin{array}{l}\text { Marken, eingetragene } \\
\text { Designs }\end{array}$ & 74 (30 Teilnahmen) & 3 (eine Teilnahme) \\
\hline \hline Gesamt & $\mathbf{4 8 9}$ (110 Teilnahmen) & $\mathbf{2 3 ~ ( 1 7 ~ T e i l n a h m e n ) ~}$ \\
\hline
\end{tabular}

Quelle: KMU Forschung Austria, N=117, bereinigt um Mehrfachnennungen

Im Instrument Einzelprojekt (BP) wurden durchschnittlich 1,28 Schutzrechte je Projekt (mit und ohne Projektpartner) angemeldet, Kooperationsprojekt: 0,82, Leitprojekt: 2,13, Wiss-Transfer: 0,24, Inno-Netz: 0,27. Patente: EP: 0,96, KP: 0,78, den höchsten Wert erzielen die Leitprojekte mit 1,38 Patenten/Projekt.

Diese Mittelwerte werden von einer geringen Zahl von Projektteilnahmen geprägt. So sind etwa in den Einzelprojekten sieben Projektteilnahmen für 115 Patente verantwortlich. Weiter wurden 84\% der nicht-technischen Schutzrechte (62) im Rahmen von Einzelprojekten angemeldet, 24 davon in nur zwei Projekten des Basisprogramms. Dies spiegelt die strategische Vorgehensweise von (vornehmlich 
Groß-)Unternehmen wider, die Patentfamilien anmelden um das unmittelbare technologischen Umfeld einer Innovation vor Konkurrenz zu schützen.

Die überwiegende Mehrheit der Schutzrechtanmeldungen erfolgte von GU. 311 der technischen und nicht-technischen Schutzrechte (60\%) wurden von GU angemeldet (1,48 je GU). Dahinter folgen die KU mit 23\% der Anmeldungen (117 Schutzrechte, 0,44 je KU) und MU mit 17\% (90 Schutzrechte, 0,43 je MU).

\section{2\% der Projekte führten zu wissenschaftlichen Publikationen}

Darüber hinaus wurden die Ergebnisse von rd. 42\% der Projekte (196, um Doppelnennungen bereinigt) wissenschaftlich publiziert. Kooperationsprojekte führten am häufigsten (73\%) und mehr als doppelt so oft als Einzelprojekte (33\%) zu wissenschaftlichen Publikationen. Die wissenschaftlichen Publikation der anderen Instrumente werden von den Instrumenten Wissenschaftstransfer (41\% bzw. 14 Publikationen) und Sondierung (32\% bzw. 11) geprägt.

\section{Abbildung 11 Wissenschaftliche Publikationen nach Instrumenten}

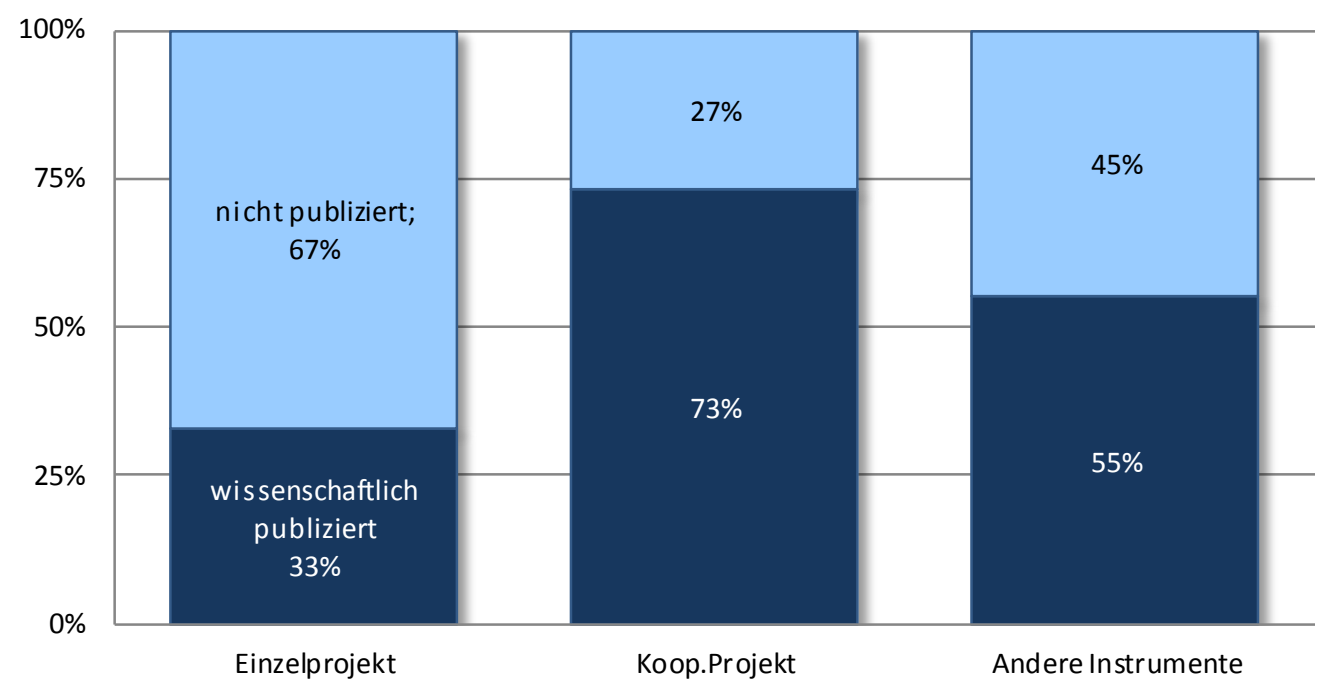

Quelle: KMU Forschung Austria, EP $=221, \mathrm{KP}=123$, Andere $=62$ Projekte; bereinigt um Mehrfachnennungen; wissenschaftlich = mit Peer Review Verfahren.

Der größte Anteil der Publikationen ist in diesem Jahr innerhalb der KU konzentriert (103) bzw. rd. 53\% aller Publikationen), gegenüber dem Vorjahr (rd. 38\%) ist dieser Wert deutlich angewachsen und ist hauptsächlich auf das verstärkte Engagement dieser (wissenschaftlich orientierten) Unternehmen in den TP zurückzuführen. Dementsprechend geringer ist der Anteil von wissenschaftlicher Publikation durch GU (75 bzw. 38\% der Publikationen) und MU (18 bzw. 9\%). 
Mit 42\% sind Produktinnovationen häufigstes Ergebnis der Forschungsvorhaben

Rd. 84\% der untersuchten Projektteilnahmen (462) führten zu mindestens einer Innovation. Die Art der Innovationen verteilen sich über die Jahre mit einer bemerkenswerte Konstanz, wenngleich der Anteil von neuen Produkten in den letzten Jahren etwas rückläufig ist, und jener von organisatorischen Innovationen leicht steigend. Dies ist wiederum auf den niedrigeren Anteil von Projekten des Instruments Einzelprojekt zurückzuführen.

Tabelle 12 Arten der Innovation von im Jahr 2011 abgeschlossenen Projekten

\begin{tabular}{|l|c|c|}
\hline Art der Innovation & Nennungen & \% von N \\
\hline Neues Produkt & 228 & $42 \%$ \\
\hline Neues Verfahren & 128 & $24 \%$ \\
\hline Verändertes Produkt & 125 & $23 \%$ \\
\hline Verändertes Verfahren & 78 & $14 \%$ \\
\hline Neues Produktdesign & 79 & $15 \%$ \\
\hline Neue Dienstleistung & 85 & $16 \%$ \\
\hline Veränderte Dienstleistung & 55 & $10 \%$ \\
\hline Organisatorische Innovation & 32 & $6 \%$ \\
\hline
\end{tabular}

Quelle: KMU Forschung Austria, N=539 Projektbeteiligungen, Mehrfachnennungen möglich

\section{Abbildung 12 Arten der Innovation von im Jahr 2011 abgeschlossenen Projekten}

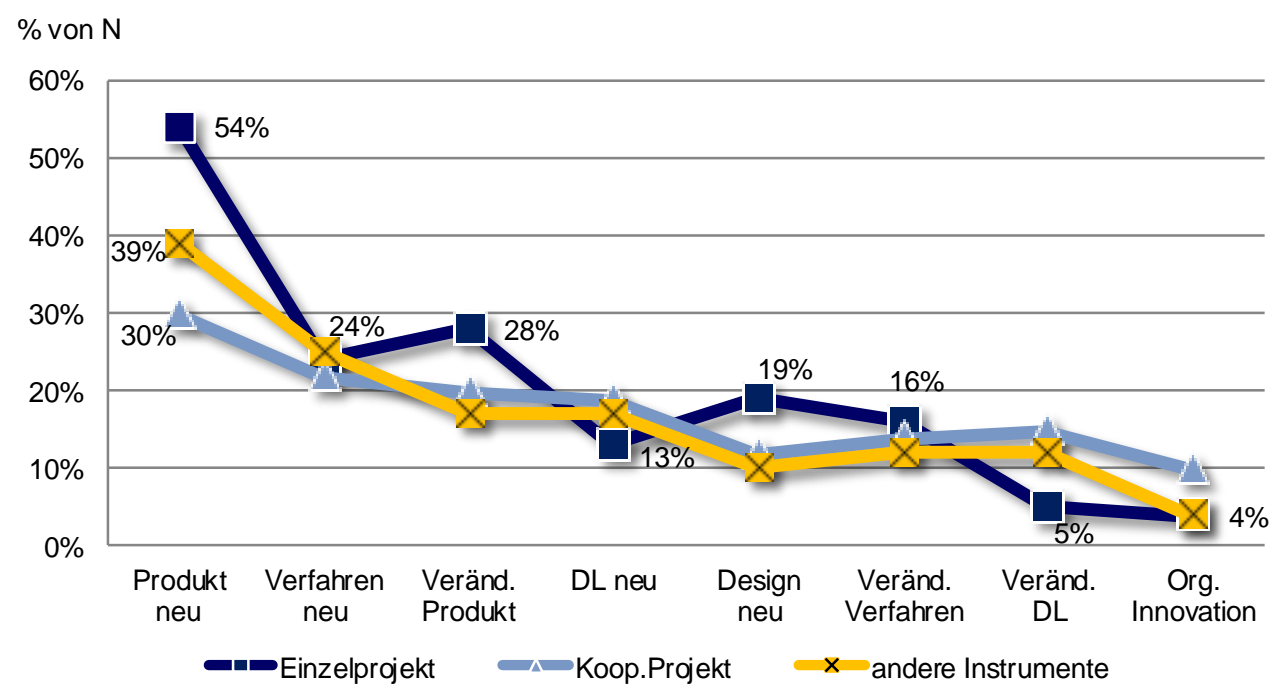

Quelle: KMU Forschung Austria, n von $\mathrm{EP}=244, \mathrm{KP}=196$, andere Instrumente=99 (Summe: 539), Mehrfachnennungen möglich 
Bei Produkt- und Designinnovationen liegen Einzelprojekte, und bei Dienstleistungen sowie organisatorische Innovationen liegen Kooperationsprojekte und tendenziell auch die weiteren Instrumente voran (siehe Abbildung).

49\% der neuen Produkte wurden von KU geschaffen, 38\% von GU und 14\% von MU. Neue und veränderte Dienstleistungen sind hingegen die vorrangige Expertise von KU (69\% bzw. 62\%). GU weisen einen überproportionalen Anteil bei den veränderten Produkten (47\%), den veränderten Verfahren (51\%) und neuen Designs $(47 \%)$ auf.

Eine weiterführende Befragung zu den Dienstleistungsinnovationen (befragt wurden nur Unternehmen, die eine neue oder veränderte DL als Projektresultat ausgewiesen haben) zielt auf die damit verbundenen Risiken ab.

Am häufigsten wurde von den Befragten die Notwendigkeit zur Kundeneinbindung als Risiko beurteilt. Auch dass die Qualität einer DL erst durch inre Anwendung feststellbar wird, ist ein relevanter Faktor.

Abbildung 13 Risiken in der Entwicklung von Dienstleistungsinnovationen

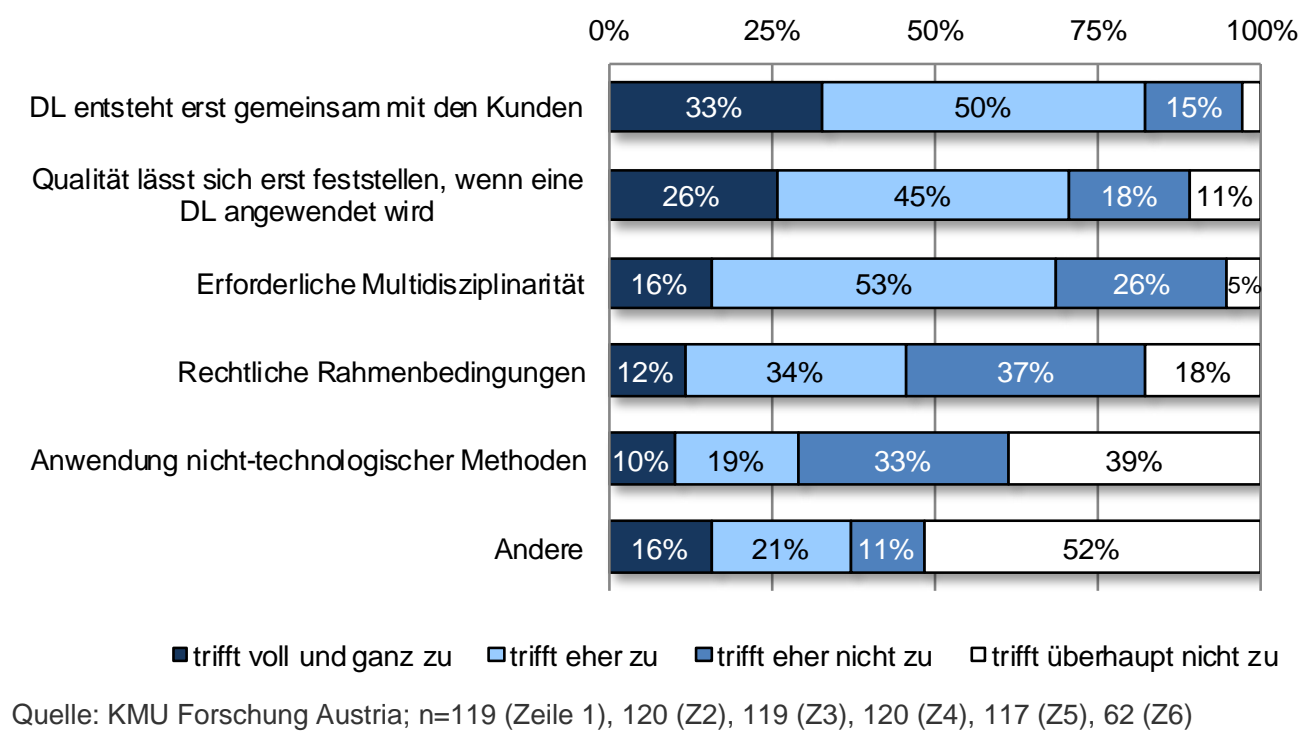

Ein erhöhtes Risiko in den Entwicklungen von DL-Innovationen ist bei den Klein- und Kleinstunternehmen zu beobachten. In sämtlichen der angeführten Kategorien dominieren sie die Antwortmöglichkeiten "trifft voll und ganz zu“ und "trifft eher zu“. Dabei schwanken ihre Anteile zwischen 50\% und $83 \%$.

Die ex-post Einschätzung der Innovationen durch die befragten Unternehmen nach deren Neuheitsgrad ergibt, dass zu einem überwiegenden Teil (62\%) Neuheiten auf internationaler Ebene erzielt werden konnten (dies impliziert sogleich auch eine nationale Neuheit bzw. eine Firmenneuheit). Bei weiteren rd. 17\% der antwortenden 
Unternehmen erwiesen sich die Innovationen als eine Neuheit auf dem österreichischen Markt, und in $21 \%$ der Fälle stellen die verwerteten Innovationsergebnisse eine Firmenneuheit dar. Der hohe Anteil internationaler Neuheiten ist ein Spiegelbild der Exportorientierung der im Monitoring enthaltenen Technologieunternehmen.

\section{In 3/4 der Fälle wird das Projektthema forschungsmäßig weiterverfolgt}

74\% der geförderten Projektteilnahmen führten zu einer Weiterverfolgung des Projektthemas (394 Beteiligungen). 27\% der Fördernehmer haben das bearbeitete Forschungsthema nach dem Projektabschluss nicht mehr weiterverfolgt bzw. für beendet erklärt. Am häufigsten werden Einzelprojekte (76\%), Kooperationsprojekte (74\%), Sondierungen $(74 \%)$ und Wissenschaftstransfers $(71 \%)$ weiterverfolgt. Von den acht Leitprojekten wurden vier weiterverfolgt. Nach Größenklassen ausgewertet liegen GU mit 77\% der Projekte leicht voran (KU: 73\%, MU: 61\%).

\section{Nationale Förderangebote ergänzen die FFG-Förderungen am häufigsten}

Neben den untersuchten Förderprogrammen hat rd. ein Drittel der Projektteilnehmer auf zusätzliche Förderangebote zurückgegriffen und damit 207 zusätzliche Förderangebote genutzt (Vorjahre: rd. 43-45\%). In 35 Fällen wurden Förderungen auf mehr als einer Ebene (regional, national, europäisch) in Anspruch genommen.

\section{Abbildung 14 Inanspruchnahme zusätzlicher Förderangebote}

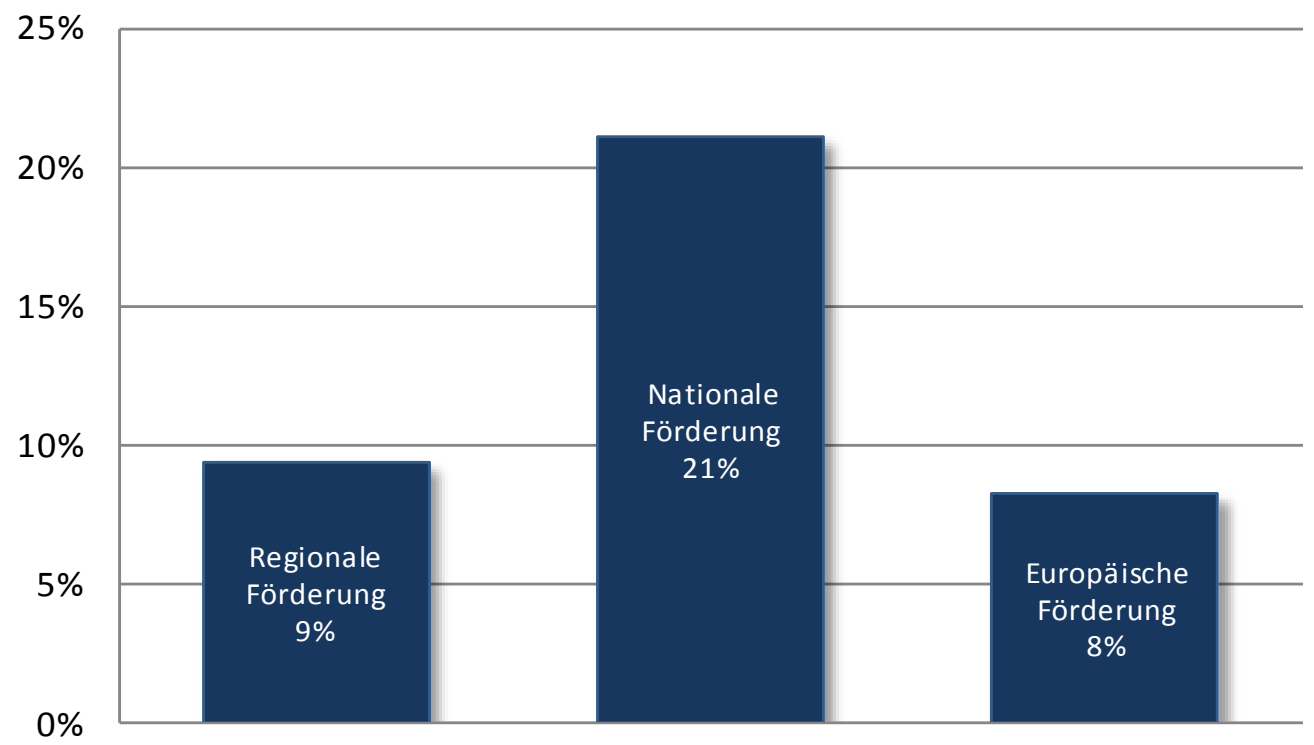

Quelle: KMU Forschung Austria, N=537

Etwas häufiger als die Einzelprojekte (31\%) haben die Kooperationsprojekte (35\%) auf weitere Förderangebote zurückgegriffen (andere: 27\%). 


\section{Projekte resultierten in Infrastrukturinvestitionen von über $€ 61$ Mio.}

Die in Zusammenhang mit der Innovationstätigkeit stehende, materielle Infrastruktur, wurde im Rahmen von 39\% der Projektteilnahmen (210) ausgebaut. Dabei konnten zu 186 Projektteilnahmen genaue Investitionsangaben getätigt werden. Diese Investitionen summieren sich auf $€ 61$ Mio., durchschnittlich ergibt sich daraus eine Investitionssumme von $€ 90.000$ (Median). Der Anteil der Infrastrukturausgaben an den Gesamtkosten der 186 Projekte liegt im Median bei 25\%. Einige der Unternehmen investierten ein Vielfaches der Projektkosten in Infrastruktur im Zuge von Produktionsumstellungen, etc.

\section{Zuwächse des technologischen Niveaus und erhöhtes Bewusstsein eigener Stärken und Schwächen}

Neben den direkten Wirkungen des Forschungsprojektes auf die Infrastruktur und das Produktportfolio zielt die Analyse auch auf die Wirkungen der geförderten Projekte auf die Innovationsfähigkeit der Unternehmen ab.

Demnach hat sich das Verständnis aktueller Technologieentwicklungen besonders verbessert, sowie das Bewusstsein eigener Stärken und Schwächen.

\section{Abbildung 15 Auswirkungen der 2011 abgeschlossenen Projekte auf unterschiedliche} Bereiche im Unternehmen

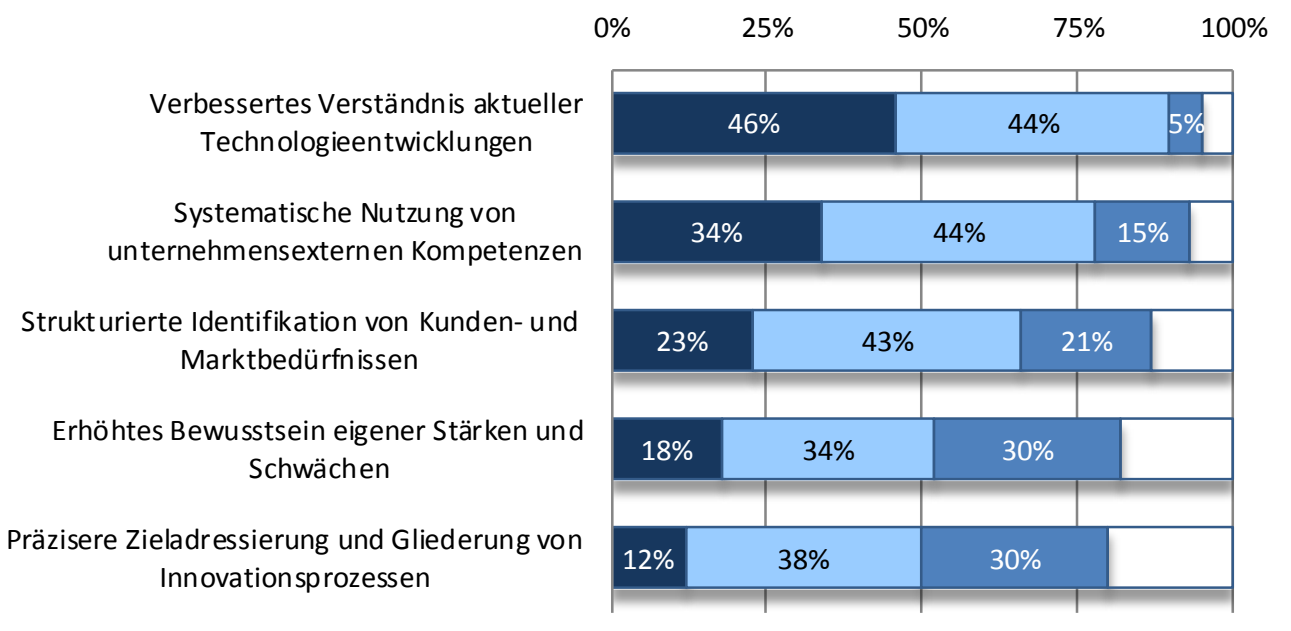

घ insgesamt verbessert $\square$ in Teilbereichen verbessert $\square$ nicht maßgeblich verändert $\square$ nicht relevant

Quelle: KMU Forschung Austria, n=540 (Z1), 539 (Z2), 535 (Z3, Z4), 537 (Z5)

KU konnten die Projekte verstärkt nutzen um Kunden- und Marktbedürfnisse zu identifizieren (70\%, MU: 57\%, GU: 65\%). 
Das Technologieverständnis konnte in rd. 94\% der Einzelprojekte verbessert werden, gegenüber $84 \%$ in den Kooperationsprojekten. Die anderen Instrumente liegen ähnlich wie die EP; nur die Markteinführungsprojekte liegen aufgrund ihrer Marktnähe deutlich darunter. Relativ ähnlich sind die Projektwirkungen in Bezug auf die Identifizierung von Kunden- und Marktbedürfnissen verteilt; nur die Markteinführungsprojekte heben sich naheliegender Weise positiv von den restlichen Instrumenten $a b$.

\section{Verbesserung der technologischen Wettbewerbspositionen bei rd. 70\%}

Sowohl auf nationaler als auch internationaler Ebene trugen insgesamt rd. 16\% der Projektteilnahmen dazu bei, dass die Unternehmen zur technologischen Spitze aufschließen konnten. Überdurchschnittlich gelang dies Unternehmen, die im Instrument Einzelprojekt aktiv waren.

Abbildung 16 Veränderung der technologischen Wettbewerbsposition

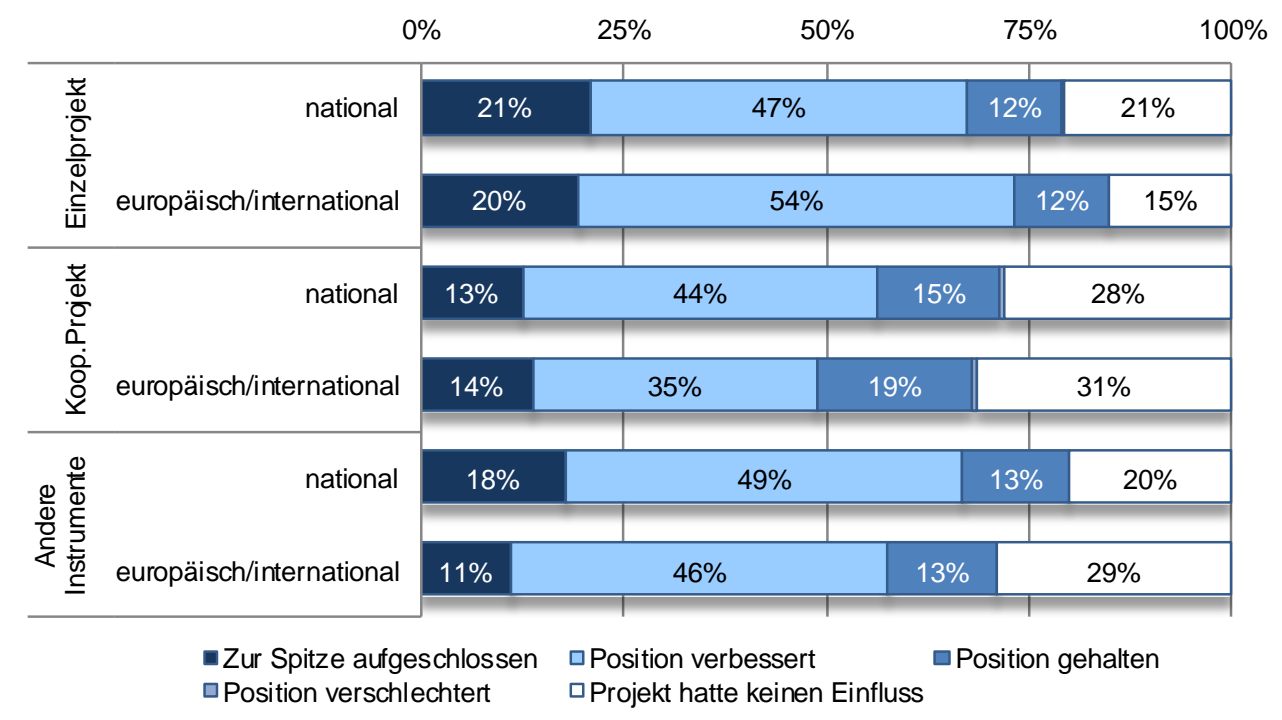

Quelle: KMU Forschung Austria, N=531

In rd. 70\% der Fälle (371) hatten die Projektteilnahmen sowohl auf nationaler wie auch internationaler Ebene eine positive Wirkung auf die Wettbewerbsposition, was wiederum spiegelbildlich für die hohe Exportorientierung der Unternehmen ist. Bei den anderen Instrumenten tun sich besonders die Innovationsnetzwerke und die Sondierungsprojekte positiv, und die Leitprojekte negativ hervor. Die Markteinführungsprojekte hatten primär Auswirkungen auf die nationale Wettbewerbsposition.

3 Projektteilnahmen $(=0,5 \%)$ gaben eine Verschlechterung der Wettbewerbsposition an. Dies ist aufgrund der Verfolgung eines untauglichen technologischen Ansatzes oder einem mit dem Projekt einhergegangenen Brain-Drain (Verlust von wissenschaftlichen MitarbeiterInnen) vorstellbar. 
3/4 der Projektteilnahmen führen zu neuen Kontakten bzw. einer Intensivierung bestehender Kontakte

Die Forschungsaktivitäten der befragten Unternehmen trugen dazu bei, dass $78 \%$ der Projektteilnahmen (425) zu neuen Kontakten führten bzw. bestehende Kontakte intensiviert wurden (Mehrfachantworten waren möglich).

In $16 \%$ der Projektteilnahmen (88) wurden weder neue Kontakte geschlossen noch bestehende Kontakte intensiviert. 54 dieser Unternehmen sind KU.

Einzelprojekte lösten in $79 \%$ der Fälle Kooperationstätigkeiten aus, da Unternehmen allein als Fördernehmer der FFG auftreten, aber dennoch oft mit wissenschaftlichen oder anderen Partnern als Subauftragnehmer kooperieren.

Die untenstehende Tabelle zeigt, dass die wichtigsten Kooperationspartner Universitäten, vor außeruniversitären Forschungseinrichtungen sowie Unternehmen entlang der Wertschöpfungskette sind. Während die Verteilung der in- und ausländischen Unternehmenspartner ähnlich verteilt ist, setzen die Fördernehmer bei wissenschaftlichen Partnern einen eindeutigen Schwerpunkt bei den heimischen Forschungseinheiten. Das Muster der Kooperationstätigkeiten entspricht jenem des Vorjahres.

Tabelle 13 Effekte auf Netzwerkbildung, 2011 abgeschlossene Projekte

\begin{tabular}{|c|c|c|c|c|}
\hline & \multicolumn{2}{|c|}{ Inland } & \multicolumn{2}{|c|}{ Ausland } \\
\hline & $\begin{array}{c}\text { neue } \\
\text { Kontakte }\end{array}$ & $\begin{array}{l}\text { bestehende } \\
\text { Kontakte }\end{array}$ & $\begin{array}{l}\text { neue } \\
\text { Kontakte }\end{array}$ & $\begin{array}{l}\text { bestehende } \\
\text { Kontakte }\end{array}$ \\
\hline Universitäten & $38 \%$ & $38 \%$ & $19 \%$ & $8 \%$ \\
\hline Fachhochschulen & $14 \%$ & $16 \%$ & $4 \%$ & $1 \%$ \\
\hline $\begin{array}{l}\text { Außeruniversitäre Forschungsein- } \\
\text { richtungen }\end{array}$ & $27 \%$ & $24 \%$ & $13 \%$ & $4 \%$ \\
\hline \multicolumn{5}{|l|}{ Andere Unternehmen, und zwar... } \\
\hline $\begin{array}{l}\text { Innerhalb der Unternehmens- } \\
\text { gruppe }\end{array}$ & $4 \%$ & $16 \%$ & $3 \%$ & $9 \%$ \\
\hline (Potenzielle) Zulieferer & $24 \%$ & $19 \%$ & $20 \%$ & $13 \%$ \\
\hline (Potenzielle) Abnehmer & $22 \%$ & $21 \%$ & $26 \%$ & $16 \%$ \\
\hline (Potenzielle) Konkurrenten & $7 \%$ & $5 \%$ & $11 \%$ & $3 \%$ \\
\hline Sonstiges & $4 \%$ & $2 \%$ & $3 \%$ & $1 \%$ \\
\hline
\end{tabular}

Quelle: KMU Forschung Austria, N=425 Unternehmen mit neuen bzw. intensivierten Kontakten

Nach Instrumenten zeigt sich aufgrund der unterschiedlichen Interventionslogiken ein heterogenes Bild: Die Neuaufnahme und Intensivierung von Kontakten mit inländischen Universitäten schwankt zwischen 32\% bei Sondierungsprojekten und 57\% bei Wissenschaftstransfers. Einzelprojekte (35\%) und Kooperationsprojekte $(40 \%)$ liegen innerhalb dieser Schwankungsbreite, wobei erstere höhere Anteile bei Kon- 
takten entlang der Wertschöpfungskette aufweisen. Bei der Kooperation mit FHs zeigt sich ein nahezu identes Bild in geringerer Intensität. Auffällig ist, dass die Einzelprojekte deutlich seltener mit außeruniversitäre Forschungsinstitutionen kooperierten (rd. 19\%) als die übrigen Instrumente (30-40\%).

Die Kontakte zu anderen Unternehmen sind ebenfalls nach diesem Muster geknüpft bzw. intensiviert worden. Die Einzel- und Kooperationsprojekte sammeln sich um die obigen Durchschnittswerte, während vor allem die Innovationsnetzwerke diese auf nationaler Ebene übertreffen. Die befragten Unternehmen arbeiten in Einzel- und Kooperationsprojekten großteils sowohl mit FE als auch Unternehmen zusammen (in ersterem Fall primär GU). Projekte des Instruments Wissenschaftstransfer bevorzugen per Definition FE und jene des Instruments Inno-Netzwerk bevorzugen Unternehmen.

\section{Zwei Drittel der Kooperationen werden in direkten Folgeprojekten weiterge- führt}

421 der Befragten haben Angaben zur Weiterführung der neuen und bestehenden Kontakte über die Projektdauer hinaus gemacht.

\section{Abbildung 17 Form der Weiterführung von neuen und bestehenden Kooperationen}

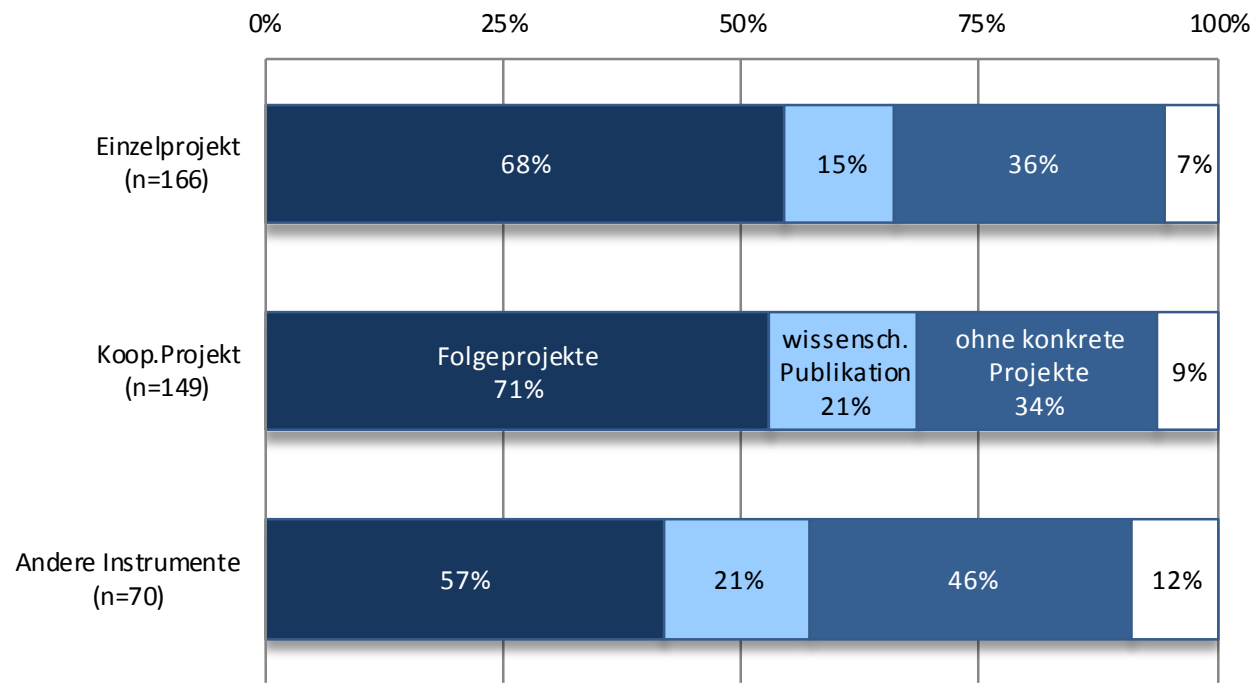

Quelle: KMU Forschung Austria, N=421 (Mehrfachantworten möglich), 4. Kategorie: nicht weitergeführt

Kooperationsprojekte und Innovationsnetzwerke werden am häufigsten in Folgeprojekten fortgesetzt $(71 \%)$, aber nur leicht vor den Einzelprojekten. Wie sich bereits oben zeigte, kooperieren Einzelprojekte relativ öfter entlang der Wertschöpfungskette. 
Ein Fünftel der Kooperationsprojekte und Innovationsnetzwerke werden in der Form von wissenschaftlichen Publikationen weitergeführt, während dieser Wert bei Einzelprojekten klar darunter liegt.

\section{Exkurs: Neukunden versus Bestandskunden}

Als Neukunden wurden Unternehmen definiert, die innerhalb der vier Jahre vor Projektende, d.h. ab dem Jahr 2007 das erste Projekt bei der FFG abgeschlossen hatten. Demnach waren $46 \%$ der befragten Unternehmen Neukunden (mit $42 \%$ der Projektteilnahmen) und 54\% Bestandskunden (mit 58\% der Projektteilnahmen). Dementsprechend sind Bestandskunden häufiger in mehreren Forschungsvorhaben gleichzeitig vertreten.

72\% der Projektteilnahmen durch Neukunden sind KU zuzurechnen, 18\% GU und $11 \%$ MU. Für knapp 18\% der Neukunden-Projektteilnahmen war das Projekt die erste FuE-Aktivität, $77 \%$ bauten hingegen ihre bisherigen FuE-Aktivitäten aus.

Damit wirkt die Portfolioerweiterung der FFG nicht nur in Richtung (kleine) FuENeulinge, sondern auch auf die Zielgruppe der durchaus FuE-intensiven Unternehmen, die bislang keine FFG Kunden waren (letztere hatten 2015 eine FuEPersonalintensität von KU: $36 \%$, MU: $24 \%$, GU: $4 \%$ ). Neukunden sind in europäischen Förderprogrammen jedoch relativ häufiger vertreten (6\% gegenüber $10 \%$ ).

Am relativ öftesten beteiligten sich Neukunden im Rahmen von Markteinführungsprojekten (71\%). Bei den Sondierungsprojekten lag der Anteil bei $48 \%$, Kooperationsprojekte: $46 \%$, Einzelprojekte: $40 \%$.

Bezüglich der Einschätzung der wirtschaftlichen Projektzielerreichung liegen Bestandskunden mit $60 \%$ über jener der Neukunden mit 50\%. Die Unterschiede bei der konkreten wirtschaftlichen Verwertungsrate bis dato sind jedoch beinahe vernachlässigbar; d.h. sie werden zwar in ähnlichem Umfang vermarktet, aber nicht so zufriedenstellend wie jene der Bestandskunden.

Der Anteil der Projektteilnahmen, die zu einer Ausweitung des Beschäftigtenstandes führten, ist nahezu ident ( $28 \%$ vs. $27 \%$ ). Dies ist insbesondere interessant, da sich Neukunden primär aus kleineren Unternehmen zusammensetzen.

Die Additionalitätsfrage ergibt, dass 37\% der Neukunden dieses Projekt ohne Förderung in keiner Form hätten umsetzen können (Bestandskunden 27\%).

Wie auch in den letzten Jahren lässt sich festhalten, dass Neukunden für die FFG einen relativ höheren Nutzen hinsichtlich der „weichen“ Innovationsfaktoren aus der FFG Forschung ableiten, aber hinsichtlich der kommerziellen Verwertung der Forschungsergebnisse hinter den Bestandskunden liegen, die bereits mehr Erfahrung mit derartigen Projekten aufweisen. 


\section{Rolle von ,Open Innovation“ im Innovations- prozess}

Dieser einmalig eingesetzte Fragenblock zielt auf die Erfahrung der Unternehmen mit unterschiedlichen Formen der Öffnung, sowie der künftigen Strategie zur Gestaltung von Innovationsprozessen ab. Hier ist zu berücksichtigen, dass Open Innovation, d.h. die Öffnung des Innovationsprozesses um nutzerorientierte (und radikalere) Lösungen zu entwickeln, kein völlig neues Phänomen darstellt. Der derzeitige Aufschwung in der politischen Diskussion ist auf die immer kürzer werdenden Innovationszyklen zurückzuführen, die auch mit dem Wunsch von Unternehmen einhergeht, eigene Ideen ev. extern schneller zu kommerzialisieren als man es selbst machen könnte, bzw. auch externe Ideen selbst zu kommerzialisieren wenn es ins eigene Portfolio passt. Darüber hinaus haben steigende FEI-Kosten bei gleichzeitigem Kostendruck weiters dazu beigetragen, um, verbunden mit einfacheren Kommunikationsmöglichkeiten via digitale soziale Netzwerke, etc. eine verstärkte akademische, und nun auch politische Diskussion zum Thema auszulösen.

In der Literatur unterscheidet man oft Unternehmen mit einer geschlossenen Innovationsstrategie, jene in Kooperation mit ausgewählten Partnern, und jene mit einer expliziten Open Innovation Strategie. Für letzteres wurden drei Kernprozesse definiert: inside-out, outside-in, sowie coupled processes.

\section{Abbildung 18 Kategorisierung von Open Innovation Prozessen}

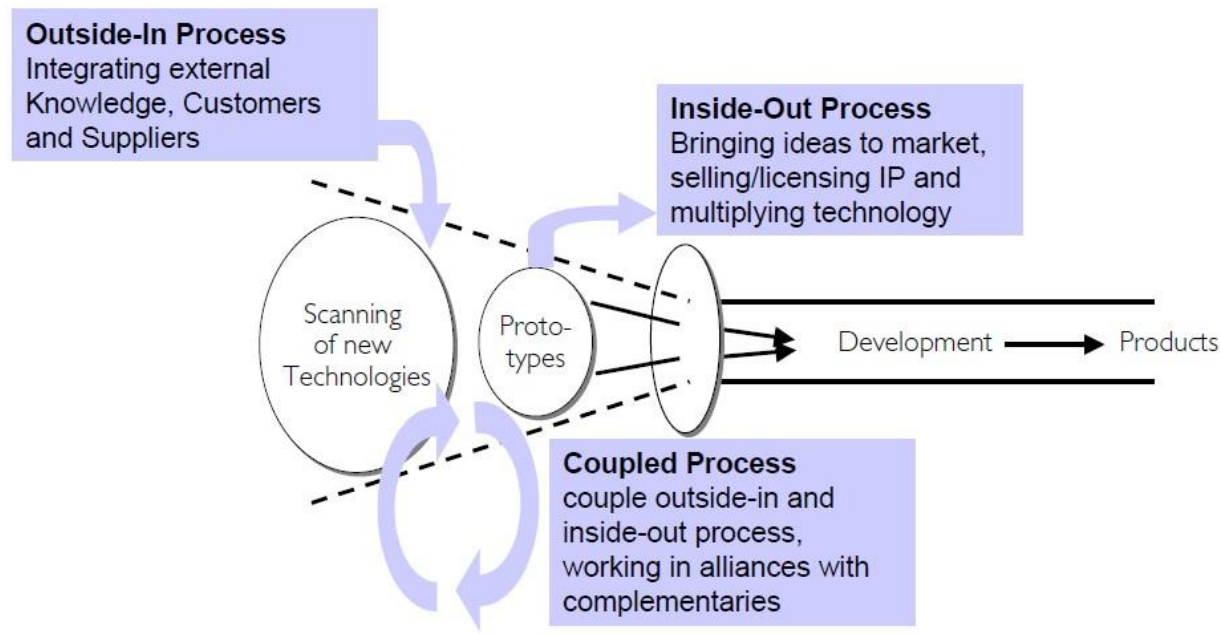

Quelle: Gassmann und Enkel (2004). Towards a Theory of Open Innovation: Three Core Process Archetypes. R\&D management conference 6, p.7 
Aufgrund dieses FFG Wirkungsmonitorings von 459 FEl-orientierten Unternehmen ist die Öffnung von Innovationsprozessen in unterschiedlichen Schattierungen durchaus ein Thema. Die folgende Abbildung veranschaulicht vereinfacht, dass diese Innovationsprozesse jedoch sehr unterschiedlich gehandhabt werden.

\section{Abnahme des Engagements mit zunehmender Tiefe der Öffnung des Inno- vationsprozesses}

Erwartungsgemäß ist eine Abnahme des Engagements mit zunehmender Tiefe der Öffnung nach außen zu beobachten. Während die Nutzung von Workshops bzw. Feedbackplattformen (outside-in) noch relativ verbreitet ist ( $41 \%$ nutzen sie zumindest oft), wird die alleinige Weitergabe von Ideen an Externe (inside-out) zur Ermöglichung bzw. Beschleunigung der Kommerzialisierung von Innovationen seltener angewendet. Dies spiegelt auch die langjährigen Ergebnisse des Wirkungsmonitorings wieder, in dem jährlich die Zahl und der Umfang von Lizenzerlösen erfasst werden.

Gemeinsame Entwicklungen im Zuge von Allianzen, Joint Ventures und Netzwerken sind noch etwas seltener, und werden am häufigsten von KU genutzt. Dass dies immerhin 14\% zumindest oft umsetzen, spiegelt wohl auch die Aktivitäten dieser Unternehmen in den Strukturprogrammen der FFG wider, die Plattformen für derartige ,coupled processes‘ bieten können.

\section{Abbildung 19 Impulse zur Öffnung von Innovationsprozessen}

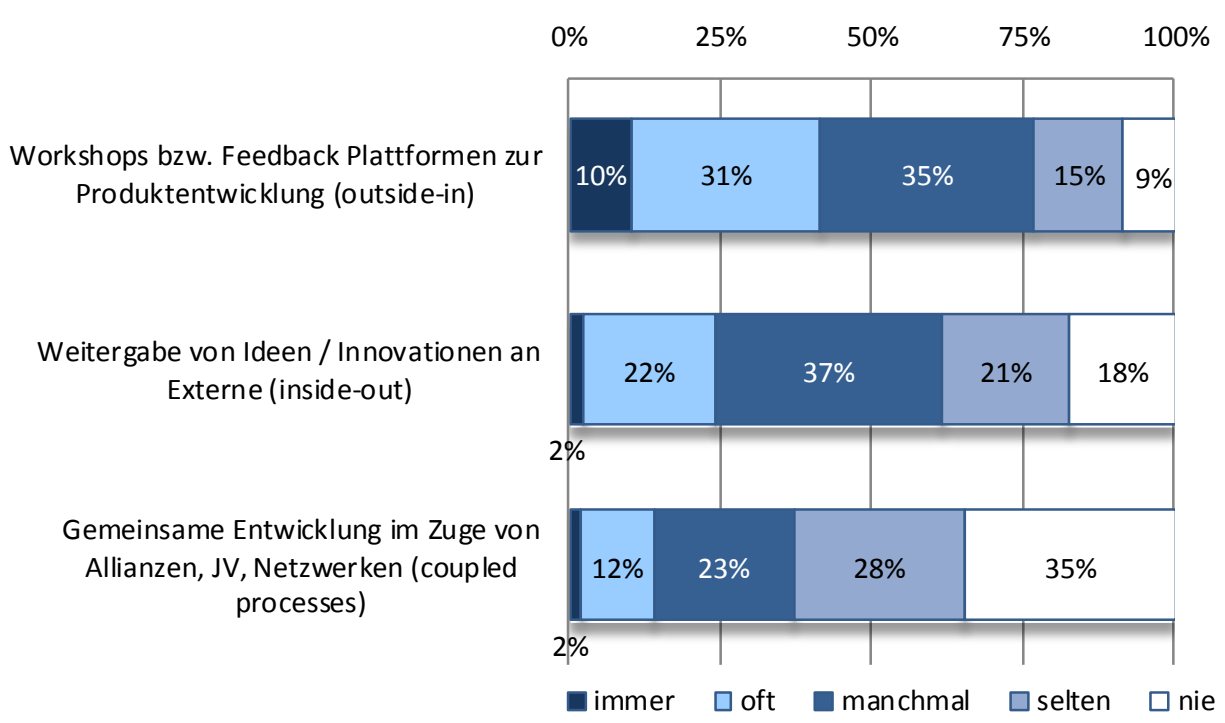

Quelle: KMU Forschung Austria, N=437 Unternehmen 
Workshops werden zu $41 \%$ zumindest oft von Kleinunternehmen genutzt (95 Unternehmen); zu 40\% (27) von MU und zu 31\% (49) von GU. Bezogen auf ÖNACE Abschnitte zeichnet sich folgendes Bild: $32 \%$ der Hersteller von Waren nutzen Workshops oft oder immer (54 Unternehmen), 49\% aus den Branchen der Information und Kommunikation (36) sowie $48 \%$ der wissenschaftlichen und technischen Dienstleistungen (53).

\section{Kunden als traditionell wichtigste Partner für den Innovationsprozess}

Bei der Einbindung externer Partner in den Innovationsprozess sind naheliegender weise insbesondere Kunden gefragt. Hier stellt sich eher die Frage, wie es sich über $10 \%$ an Unternehmen leisten können, ihre Kunden kaum in den Innovationsprozess einzubeziehen. $43 \%$ greifen mehr oder weniger regelmäßig auf externe Spezialisten zurück und rd. $36 \%$ auf Zulieferer. Unter der Kategorie "Sonstige“ wurden primär wissenschaftliche Partner aus dem Bereich der Universitäten und FHs beschrieben.

\section{Abbildung 20 Einbindung externer Partner in den Innovationsprozess}

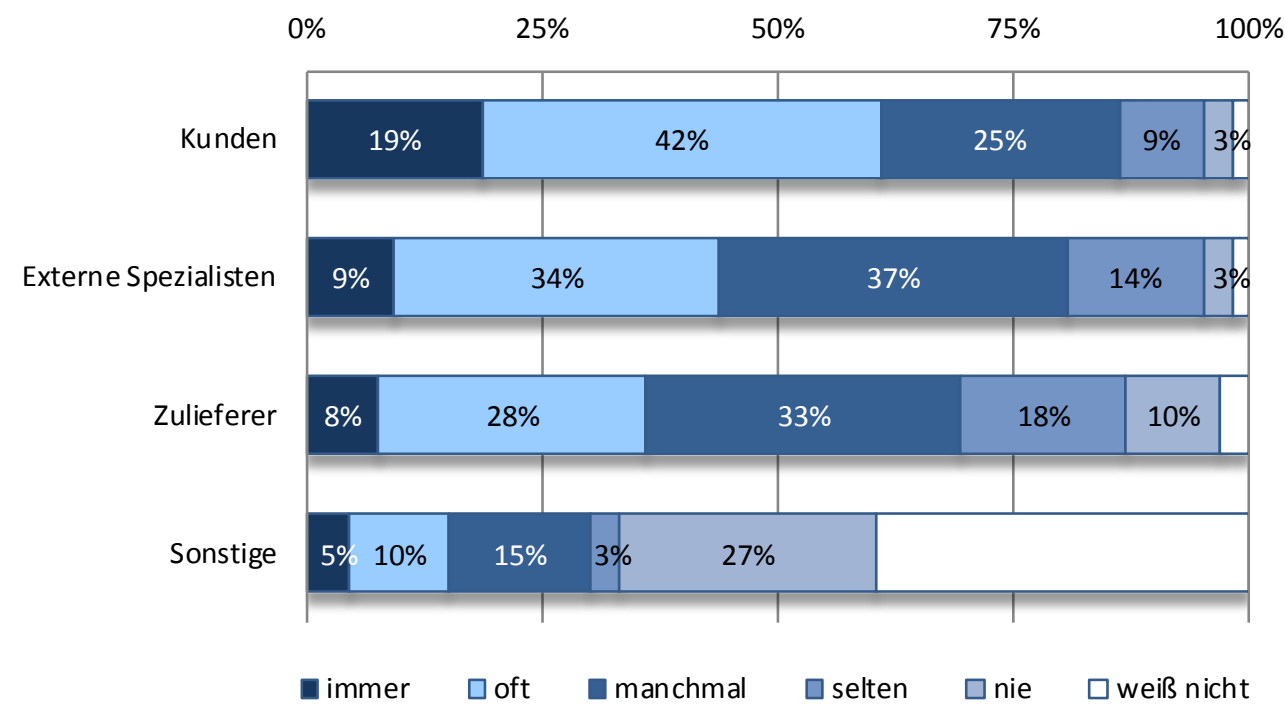

Quelle: KMU Forschung Austria; N=445 Unternehmen

Zulieferer werden von GU mit $42 \%$ am häufigsten zumindest öfters einbezogen (KU: 28\%, MU: 39\%). Ähnlich verhält es sich mit externen Spezialisten, die von knapp der Hälfte $(46 \%)$ der antwortenden GU zumindest oft herangezogen werden (KU: $41 \%$; MU: $42 \%)$.

Nach Branchen ausgewertet zeigt sich, dass die Einbeziehung von Kunden im Bereich Information und Kommunikation am häufigsten vorkommt (65\% bzw. 48 Unternehmen). Wissenschaftliche und technische Dienstleister binden in $61 \%$ der Fälle (68) und Hersteller von Waren in 60\% ein (100). Letztere greifen dafür häufiger auf ihre Zulieferer zurück (44\%, 73 Unternehmen), die im Bereich IKT eine eindeutig 
geringe Rolle spielen (18\%, 13 Unternehmen; wissenschaftl. DL 32\%, 36). Externe Spezialisten werden von $48 \%$ der wissenschaftlichen DL (53), $42 \%$ der Hersteller von Waren (70) und 32\% der IKT Unternehmen (24) eingesetzt.

\section{Für wen ist die zukünftige Öffnung des Innovationsprozesses eine Option?}

Da im Wirkungsmonitoring ein relevanter Ausschnitt von (sehr) FEl-aktiven Unternehmen im Sample enthalten ist, stellt sich die Frage nach deren zukünftigen Strategie. Immerhin knapp ein Fünftel der Teilnehmer (100 Unternehmen) geben an, dies auf jeden Fall machen zu wollen und weitere 45\% (246) halten fest, dass eine weitere Öffnung durchaus möglich sei. Umgekehrt halten rd. ein Viertel der Unternehmen eine Öffnung für zumindest wenig wahrscheinlich.

\section{Abbildung 21 Planen die Unternehmen den Innovationsprozess künftig stärker zu öffnen?}

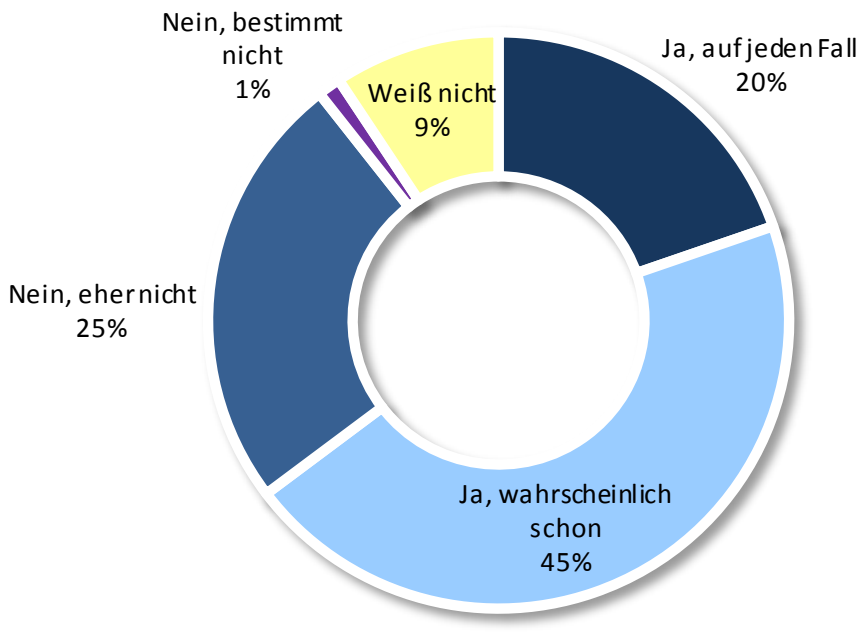

Quelle: KMU Forschung Austria, N=451 Unternehmen

Knapp ein Viertel der befragten KU (23\%) gibt an, Innovationsprozesse künftig auf jeden Fall stärker öffnen zu wollen (MU: 12\%, GU: 29\%).

Etwas seltener als in der Gesamtverteilung vertreten, planen Unternehmen aus dem ÖNACE Abschnitt Herstellung von Waren künftig stärker („auf jeden Fall“) zu öffnen (zu 17\%), während Unternehmen aus dem Bereich der wissenschaftlichen und technischen Dienstleistungen mit 23\% eine verhältnismäßig stärkere Öffnung anstreben. 
Unternehmen mit einer geringen Exportquote $(<25 \%)$ neigen mit $27 \%$ eher dazu, den Innovationsprozess künftig stärker öffnen zu wollen. Umgekehrt wollen Unternehmen mit einer hohen Exportquote (> 75\%) nur zu 16\% den Innovationsprozess jedenfalls stärker öffnen. Dieses Antwortverhalten legt die Hypothese nahe, dass die Intensität der Konkurrenzsituation bzw. die Nähe zu den Kunden die Einstellung der Unternehmen zu diesem Thema beeinflusst.

Zusammenfassend können wir festhalten, dass Open Innovation bei den FEl-aktiven Unternehmen durchaus, aber selektiv auf Interesse stößt. Die Neigung zur Öffnung des Innovationsprozesses hängt von strukturellen Markmalen der Unternehmen ab, der Branche bzw. dem Technologiefeld, in dem sie tätig sind, sowie der Zielmärkte und damit zusammenhängend der Intensität der Konkurrenzsituation bzw. mit der Kundennähe. Diese deskriptiven Ergebnisse werden auch durch das Resultat einer multinominalen Regression unterstützt, die insbesondere die branchenmäßige/technologische Verankerung der Unternehmen sowie die Exportabhängigkeit als Determinanten hervorstreicht. 


\section{Additionalität}

Die Frage nach der Projekt-Additionalität der Förderung, bzw. dem Mitnahmeeffekt, gibt Auskunft darüber, in welchem Ausmaß Projekte ohne Erhalt der Förderung durchgeführt worden wären.

\section{Abbildung 22 Hätten Sie das Projekt auch ohne Förderung durchgeführt?}

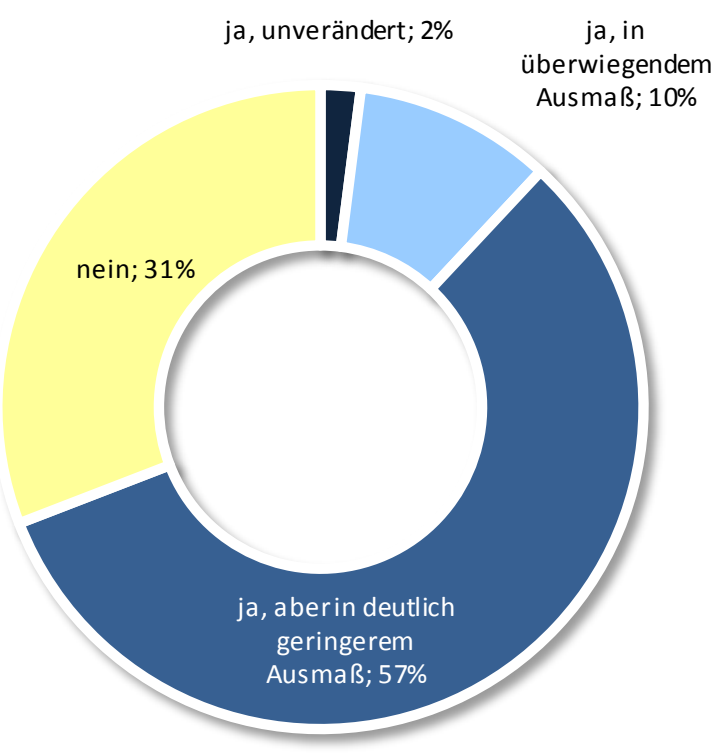

wenn ja, dann zeitlich verzögerte Umsetzung: $85 \%$

wenn ja, dann ohne /weniger Kooperationspartner: $48 \%$

Quelle: KMU Forschung Austria, N=544 Projektteilnahmen mit Projektende 2011

Die $31 \%$ an Fällen, die ohne Förderung nicht umgesetzt worden wären, bedeuten gegenüber dem Vorjahr einen Anstieg um rd. 5 Prozentpunkte. Dies ist hauptsächlich auf den Anstieg an Projekten über das Basisprogramm hinaus zurückzuführen. Aber auch im Basisprogramm ist die Tendenz leicht steigend, wie die untenstehende Abbildung zeigt.

Während Projekte, die Bausteine in einem bestehenden Aktivitätsfeld waren, etwas häufiger an der Umsetzung festgehalten hätten (27\% hätten es nicht durchgeführt), ist der Einstieg in ein neues Aktivitätsfelder tendenziell häufiger von der Förderung abhängig (37\% hätten es nicht durchgeführt).

Bezüglich der Innovationsart zeigt sich, dass neue (37\%) oder veränderte (35\%) Dienstleistungen am häufigsten von Förderungen abhängig waren. Neue Verfahren $(30 \%)$ und Produkte $(26 \%)$ wären im Falle keiner Förderung eher durchgeführt worden (organisatorische Innovationen 34\%, Designinnovationen 19\%). 
Rd. 64\% wären nicht in der Lage gewesen, die notwendigen finanziellen Mittel zu lukrieren, 22\% hätten das technische oder wirtschaftliche Projektrisiko als zu hoch erachtet, und weitere 14\% der Befragten nannten andere Gründe. Darunter etwa der notwendige Kooperationsbedarf für das Projekt oder die Projektinitiative seitens der Konsortialpartner.

\section{Geringere Mitnahmeeffekte als in den vorherigen Untersuchungsjahren}

In lediglich $2 \%$ der Teilprojekte (11) kam es zu einem vollständigen Crowding-out privater Innovationsausgaben durch die Förderung des Forschungsprojekts, was aufgrund der Ergebnisse der letzten Jahre eine klare Tendenz nach unten bedeutet (siehe nachfolgende Abbildung). 10\% der untersuchten Forschungsprojekte wären zumindest in überwiegendem Ausmaß vorangetrieben worden (Tendenz ebenso nach unten).

Weitere wesentliche Wirkungen einer fehlenden Förderung wären die zeitliche Verzögerung der Projekte und die Verringerung des Umfangs. Während erstere jedes Jahr bei $80-86 \%$ der Projektteilnahmen liegt, die bei fehlender Förderung trotzdem vorangetrieben worden wären, beläuft sich die Verringerung des Umfangs jährlich auf 54-60\%. Bei einer Auswertung allein nach Basisprogramm wäre die Bandbreite noch geringer.

\section{Instrumente mit höherer Marktferne bzw. Risiken zeigen höhere Additionalität der Förderung}

Sondierungsprojekte (44\%), Kooperationsprojekte (44\%), Wissenschaftstransfers (40\%) und Innovationsnetzwerke (38\%) wären ohne Förderung überdurchschnittlich oft nicht durchgeführt worden. Diese Abweichungen zu den Einzelprojekten (18\%) ist in erster Linie durch die jeweilige Instrumentenlogik mit den assoziierten Risiken begründbar.

Hinsichtlich der Unternehmensgrößen zeigen sich, wie in den vergangenen Jahren, zu erwartende Muster: je kleiner das Unternehmen, desto geringer die Fähigkeit bzw. Bereitschaft der Projektdurchführung ohne Fördermittel (KU 47\% keine Projektdurchführung, MU 29\%, GU 14\%). 
Abbildung 23 Additionalität der Projektteilnahmen mit Projektende 2007-2011

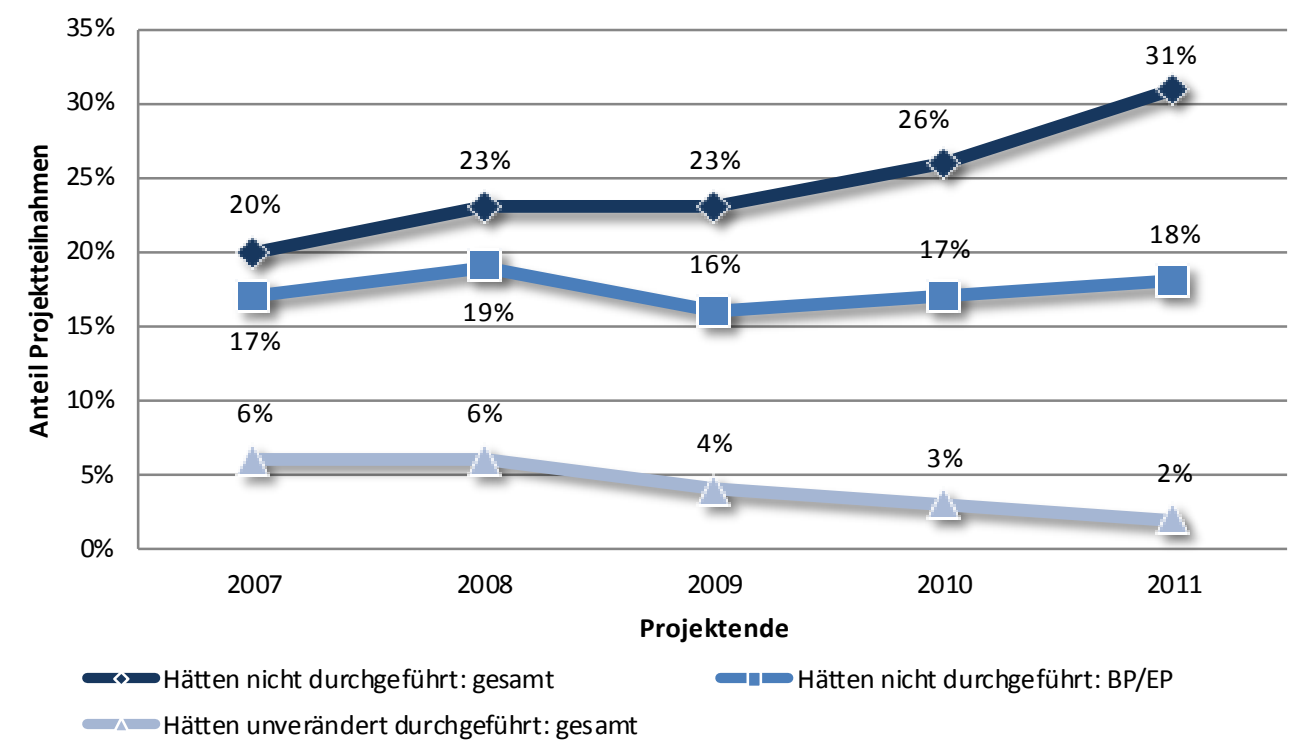

Quelle: KMU Forschung Austria; BP=Basisprogramm, EP=Instrument Einzelprojekt (ab 2011)

Die Kurve für die Projekt-Additionalität für das gesamte Portfolio (,hätten nicht durchgeführt') steigt insbesondere aufgrund der über die Jahre zunehmenden Zahl an Projekten aus dem FFG Portfolio, die über das Basisprogramm (nun im Instrument Einzelprojekt) hinausgehen. Diese zeigen eine etwas höhere ProjektAdditionalität, da sie tlw. nicht so nahe am Markt positioniert sind. Aber auch die separate Auswertung für das Basisprogramm (bzw. Einzelprojekt ab Projektende 2011) zeigt eine leicht steigende Tendenz. Demensprechend ist der Anteil an Projekten, die ohne Förderung unverändert durchgeführt worden wären, über die Jahre gesunken. Inwieweit dies auf die konjunkturelle Entwicklung der letzten Jahre bzw. administrative Aspekte innerhalb der FFG zurückzuführen ist, kann leider nicht getrennt dargestellt werden. Aus den restlichen Teilen des Wirkungsmonitorings können wir jedoch ableiten, dass sich die Unternehmen aufgrund des wirtschaftlichen Umfeldes nach der Wirtschaftskrise nach wie vor etwas schwer tun, mit ihren Innovationen Umsatzvolumina in der Höhe des Vor-Krisenniveaus zu erreichen. Dies belastet natürlich auch die FEI-Aktivitäten der Unternehmen; in solchen Zeiten steigt die Relevanz der Aktivitäten von Innovationsagenturen über das normale Maß. 


\section{Wirkungen bei Forschungseinheiten}

\section{Methodik und Eckdaten der analysierten Forschungseinheiten}

Das Wirkungsmonitoring der FFG Förderung beinhaltet auch in diesem Jahr wieder die Projektteilnahmen der involvierten Forschungseinrichtungen bzw. deren Institute/Forschungseinheiten (FE). Aufbauend auf die Befragung des Vorjahres wurde ein leicht adaptierter Fragebogen im Zuge einer Onlinebefragung an die geförderten Forschungseinrichtungen (Universitätsinstitute - UNI, Fachhochschulen - FH sowie außeruniversitäre Forschungseinrichtungen - AUF) versendet. Auf Basis der EmailAdressen der FFG Datenbank wurden die Fragebögen zunächst an die hauptverantwortlichen technischen Projektleiterlnnen übermittelt. Wenn eine Email-Adresse nicht mehr zugestellt werden konnte, wurden - soweit vorhanden - deren Vorgesetzte, und als dritte Präferenz die kaufmännischen Ansprechpersonen angeschrieben. Bei Nichtverfügbarkeit alternativer Adressen wurden die Einladungen zur Teilnahme an allgemeine Unternehmensadressen (z.B. Sekretariate) mit der Bitte um Weiterleitung verschickt.

Von der FFG wurden Daten zu insgesamt 662 Projektteilnahmen von Forschungseinrichtungen zur Verfügung gestellt. Bereinigt nach den bewährten Kriterien des Wirkungsmonitorings der Unternehmen (siehe dort), bzw. dem Ausschluss nicht relevanter Instrumentengruppen, verblieben 446 Forschungsbeteiligungen, die angeschrieben wurden. Weitere 30 Projektteilnahmen mussten aufgrund von z.B. der Schließung von Instituten, Doppelnennungen, Ausstieg während der Projektteilnahme, und 13 Fragebögen aufgrund von Nicht-Zustellbarkeit ausgeschieden werden (bei der ersten Aussendung konnten noch 123 Emails nicht zugestellt werden). Damit hat der Fragebogen letztlich in 403 Fällen (rd. 91\% der Grundgesamtheit) die jeweiligen Adressaten erreicht.

Von den ursprünglich 446 bzw. dann 403 Adressaten beantworteten 192 den Fragebogen. Das entspricht einer Netto-Rücklaufquote von rd. 48\% (Vorjahr: 50\%). Die 192 analysierten Projektteilnahmen wurden in 163 unterschiedlichen Projekten von 118 verschiedenen FE umgesetzt (FFG Firmennummer als Alleinstellungsmerkmal).

Im klaren Unterschied zur Unternehmensbefragung liegt bei der Befragung der FE der Schwerpunkt der Antworten nicht beim Basisprogramm (4\%), sondern bei den Thematischen Programmen (67\%), gefolgt von BRIDGE (15\%) und den Strukturprogrammen (12\%). Der Grund liegt darin, dass Forschungseinrichtungen im Basisprogramm nur als Projektnehmer aufscheinen, wenn sie eigene kommerzielle Interessen verfolgen, d.h. ähnlich wie Unternehmen agieren. Im Normalfall treten im Basis- 
programm nur Unternehmen als Fördernehmer der FFG auf, und Forschungsinstitute werden als Sub-Vertragsnehmer der Unternehmen tätig. In letzterem Fall hat die FFG keine Daten zu den involvierten Forschungseinheiten in ihrer Monitoring Datenbank. Die Schwerpunkte der im Sample enthaltenen Programmbeteiligungen sind dementsprechend bei Neue Energien, BRIDGE, IV2S(plus), FIT-IT sowie COIN.

Tabelle 142011 abgeschlossene Projekte; Rücklauf der online befragten Forschungseinheiten nach Programmbeteiligung

\begin{tabular}{|c|c|c|c|c|c|c|}
\hline $\begin{array}{l}\text { Be- } \\
\text { reich }\end{array}$ & Programm & $\begin{array}{c}\text { Ver- } \\
\text { sendet }\end{array}$ & $\begin{array}{l}\text { Erreicht } \\
\text { und } \\
\text { relevant }\end{array}$ & $\begin{array}{c}\text { Aus- } \\
\text { gewertet }\end{array}$ & $\begin{array}{l}\text { Netto- } \\
\text { Rücklauf }\end{array}$ & $\begin{array}{c}\text { Anteil an } \\
\text { ausgewer- } \\
\text { teten Fra- } \\
\text { gebögen }\end{array}$ \\
\hline \multirow{3}{*}{ BP } & BRIDGE & 57 & 53 & 28 & $53 \%$ & $15 \%$ \\
\hline & Basisprogramm & 24 & 20 & 8 & $40 \%$ & $4 \%$ \\
\hline & EUROSTARS & 3 & 3 & 0 & - & - \\
\hline \multirow{11}{*}{ TP } & Neue Energien 2020 & 110 & 104 & 52 & $50 \%$ & $27 \%$ \\
\hline & IV2S(plus) & 71 & 64 & 23 & $36 \%$ & $12 \%$ \\
\hline & FIT-IT & 38 & 36 & 19 & $53 \%$ & $10 \%$ \\
\hline & $\mathrm{EdZ}$ & 21 & 17 & 9 & $53 \%$ & $5 \%$ \\
\hline & KIRAS & 13 & 11 & 6 & $55 \%$ & $3 \%$ \\
\hline & TAKE OFF & 14 & 13 & 6 & $46 \%$ & $3 \%$ \\
\hline & NANO & 22 & 16 & 6 & $38 \%$ & $3 \%$ \\
\hline & benefit & 10 & 10 & 4 & $40 \%$ & $2 \%$ \\
\hline & NAWI & 6 & 6 & 2 & $33 \%$ & $1 \%$ \\
\hline & GEN-AU & 5 & 3 & 2 & $67 \%$ & $1 \%$ \\
\hline & AT:net & 2 & 0 & 0 & - & - \\
\hline \multirow{3}{*}{ SP } & COIN & 22 & 21 & 13 & $62 \%$ & $7 \%$ \\
\hline & $\begin{array}{l}\text { Research Studios } \\
\text { Austria }\end{array}$ & 16 & 16 & 10 & $63 \%$ & $5 \%$ \\
\hline & FHplus & 2 & 0 & 0 & - & - \\
\hline \multirow[t]{2}{*}{ ALR } & ASAP & 10 & 10 & 4 & $40 \%$ & $2 \%$ \\
\hline & Gesamt & 446 & 403 & 192 & $48 \%$ & $100 \%$ \\
\hline
\end{tabular}

Quelle: KMU Forschung Austria; BP = Bereich Basisprogramme, TP = Thematische Programme, $\mathrm{SP}=$ Strukturprogramme, ALR = Agentur für Luft- und Raumfahrt 
Nach Organisationsart liegt der Schwerpunkt bei Universitäten und außeruniversitären Forschungsinstituten.

Tabelle 152011 abgeschlossene Projekte; Rücklauf der online befragten Forschungseinheiten nach Organisationsart

\begin{tabular}{|l|c|r|r|r|r|c|}
\hline Organisationsart & $\begin{array}{c}\text { Ver- } \\
\text { sendet }\end{array}$ & $\begin{array}{c}\text { Erreicht } \\
\text { und } \\
\text { relevant }\end{array}$ & $\begin{array}{c}\text { Anteil } \\
\text { erreicht }\end{array}$ & $\begin{array}{c}\text { Ausge- } \\
\text { wertet }\end{array}$ & $\begin{array}{c}\text { Netto- } \\
\text { Rück- } \\
\text { lauf }\end{array}$ & $\begin{array}{c}\text { Anteil an } \\
\text { ausgewer- } \\
\text { teten Fra- } \\
\text { gebögen }\end{array}$ \\
\hline Universitätsinstitute & 202 & 183 & $91 \%$ & 89 & $49 \%$ & $46 \%$ \\
\hline Fachhochschulen & 26 & 24 & $92 \%$ & 12 & $50 \%$ & $6 \%$ \\
\hline $\begin{array}{l}\text { Außeruniversitäre } \\
\text { Forschungsinstitute }\end{array}$ & 182 & 165 & $91 \%$ & 77 & $47 \%$ & $40 \%$ \\
\hline Kompetenzzentren & 23 & 21 & $91 \%$ & 11 & $52 \%$ & $6 \%$ \\
\hline Sonstige & 13 & 10 & $77 \%$ & 3 & $30 \%$ & $2 \%$ \\
\hline \hline Gesamt & 446 & 403 & & 192 & $48 \%$ & $100 \%$ \\
\hline
\end{tabular}

Quelle: KMU Forschung Austria; * = inklusive Privatuniversitäten; Kooperative FE ist eine FFG-interne Klassifikation, die nicht mit ACR Instituten verwechselt werden sollte

Die Zuordnung der Teilprojekte nach den Förderungs- und Finanzierungsinstrumenten der FFG zeigt einen überwältigenden Schwerpunkt beim Instrument Kooperationsprojekt. Deshalb wird auf die Auswertung nach Instrumenten analog zur Unternehmensbefragung verzichtet. Vielmehr werden Resultate nach Programmbereichen bzw. einzelnen Programmen dargestellt.

Tabelle 162011 abgeschlossene Projekte; Instrumentenzuordnung

\begin{tabular}{|l|l|c|c|}
\hline Förderinstrument & \multicolumn{1}{|c|}{ Zuordenbare Programme } & Anzahl & Anteil \\
\hline C3 Einzelprojekt & Basisprogramm & 8 & $4 \%$ \\
\hline C4 Kooperationsprojekt & $\begin{array}{l}\text { ASAP, benefit, Energie der Zukunft, } \\
\text { GEN-AU, FIT-IT, IV2Splus, KIRAS, } \\
\text { NAWI, NANO, Neue Energien } \\
\text { 2020, TAKE OFF }\end{array}$ & 131 & $68 \%$ \\
\hline C5 Leitprojekt & $\begin{array}{l}\text { Energie der Zukunft, Neue Ener- } \\
\text { gien 2020 }\end{array}$ & 2 & $1 \%$ \\
\hline C6 Wissenschaftstransfer & BRIDGE & 28 & $15 \%$ \\
\hline C9 Strukturaufbau & Research Studios Austria, COIN & 11 & $6 \%$ \\
\hline C10 Innovationsnetzwerk & COIN & 12 & $6 \%$ \\
\hline \hline Gesamt & & 192 & $100 \%$ \\
\hline
\end{tabular}

Quelle: KMU Forschung Austria 


\section{Initiierung und Positionierung der Projekte im Portfolio der Forschungseinheiten}

Der Impuls zur Durchführung der Projekte ist in knapp 46\% der Fälle von den FE selbst ausgegangen. Insgesamt zeigen sich die FE heuer etwas initiativer (Vorjahr: $39 \%$ ). Dies ist hauptsächlich auf den höheren Anteil von Projekten aus dem Programm Neue Energien zurückzuführen, die mit rd. 70\% Projektideen aus dem eigenen Forschungsinstitut umsetzten.

Bei rd. $28 \%$ war die Projektdurchführung eine gemeinsame Initiative, und bei weiteren $23 \%$ konnte diese eindeutig dem Unternehmenssektor zugeordnet werden. Die gemeinsame Definition von Projektideen erfolgte besonders in Fachhochschulen, KZentren und außeruniversitären FE, die tendenziell stärker mit Unternehmen zusammenarbeiten. Bei den Universitäten entsprechen die Antwortkategorien nahezu der Gesamtverteilung.

Abbildung 24 Woher kam der Impuls für das Projekt

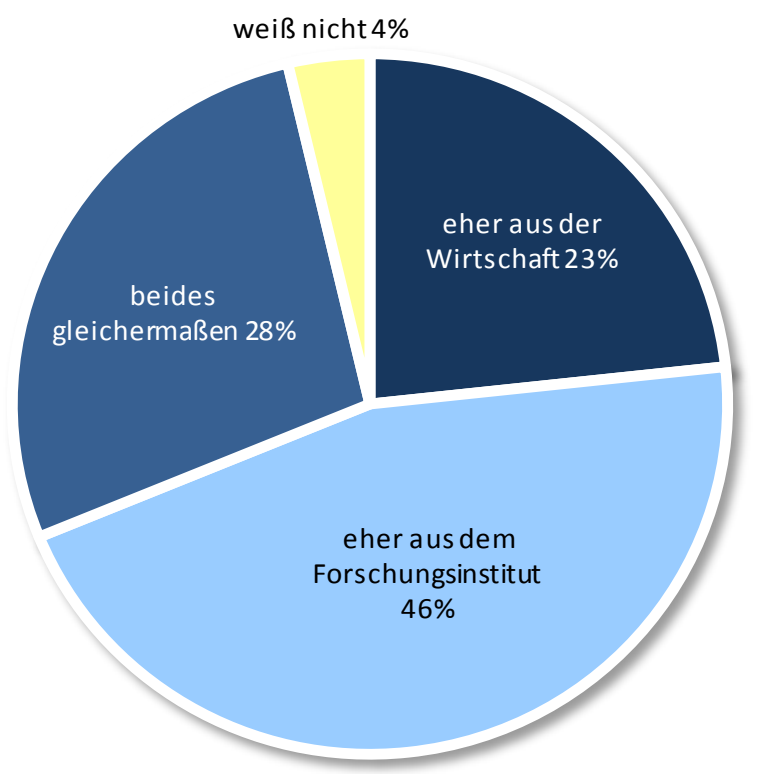

Quelle: KMU Forschung Austria; N=192 Projektbeteiligungen durch Forschungseinheiten

Der hohe Anteil an unmittelbar zuordenbaren Vorprojekten betont die kontinuierliche und kumulative Natur der Forschung in den FE

Wie im Vorjahr konnten 61\% der Projektteilnahmen inhaltlich zumindest einem FuEVorprojekt in der FE zugeordnet werden. Im Bereich TP wurden rd. 40\% der Projekte ohne unmittelbare Vorprojekte gestartet. Im restlichen FFG Portfolio ist der Anteil deutlich geringer. 
Abbildung 25 Zuordnung zu unmittelbaren FuE-Vorprojekten

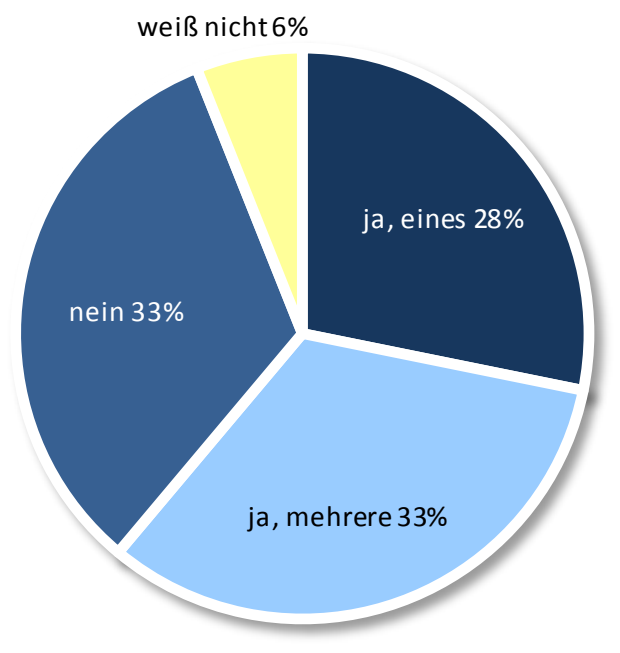

Quelle: KMU Forschung Austria; N=190 Projektteilnahmen

$80 \%$ der Vorprojekte wurden durch Steuergeld unterstützt (Vorjahr: 84\%).

Dies erfolgte ebenfalls überwiegend durch die FFG, rd. drei Viertel $(78 \%)$ der relevanten Fördernehmer haben im Vorfeld ein FFG-Projekt abgewickelt. In 30\% der Fälle wurden Mittel der Europäischen Kommission, Landesförderungen, oder des FWF in Anspruch genommen. 10 dieser Projektteilnahmen haben im Vorfeld auf mehrere Finanzierungsquellen zurückgegriffen.

44\% der relevanten Projektteilnehmer geben an, dass das Vorprojekt von einem Unternehmen (mit-)finanziert wurde (Vorjahr: 30\%) und bei 27\% der Vorprojekte erfolgte zu einem bestimmten Ausmaß eine Eigenfinanzierung durch die Institute (Vorjahr: 28\%).

\section{Die Projekte begründen zu einem Drittel neue thematische Schwerpunkte an den Instituten}

Auch die folgende Tabelle veranschaulicht, dass die Projekte in den Forschungseinrichtungen zu einem wesentlichen Anteil bereits in einen thematischen Schwerpunkt des Instituts subsumiert werden konnten (99 von 189 Projekten bzw. 52\%; Vorjahr: 60\%). Weitere 33\% (Vorjahr: 35\%) wurden als Grundlage für einen neuen thematischen Institutsschwerpunkt interpretiert.

Der Anteil von 13\% an Projekten, die inhaltliche Randbereiche des Instituts darstellen und aufgrund dessen eher keine weiterführenden Aktivitäten am Institut nach sich ziehen werden, ist erfreulicherweise niedrig, hat sich aber gegenüber dem Vorjahr erhöht (7\%). 
Tabelle 17 Verortung der Projekte im Institutsprofil

\begin{tabular}{|c|c|c|c|c|c|c|c|}
\hline & \multicolumn{6}{|c|}{ War das Projekt an Ihrem Institut ein ... } \\
\hline & & \multicolumn{2}{|c|}{$\begin{array}{l}\text { Singuläres } \\
\text { Projekt }\end{array}$} & \multicolumn{2}{|c|}{$\begin{array}{l}\text { Teil eines } \\
\text { größeren } \\
\text { Vorhabens }\end{array}$} & \multicolumn{2}{|c|}{ Gesamt } \\
\hline & & Anzahl & $\%$ & Anzahl & $\%$ & Anzahl & $\%$ \\
\hline \multirow{5}{*}{ 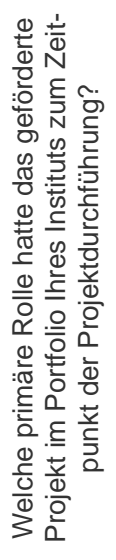 } & $\begin{array}{l}\text { Das Projekt war Teil } \\
\text { eines bestehenden the- } \\
\text { matischen Schwer- } \\
\text { punkts, mit einer Mehr- } \\
\text { zahl an Projekten }\end{array}$ & 62 & $48 \%$ & 37 & $62 \%$ & 99 & $52 \%$ \\
\hline & $\begin{array}{l}\text { Das Projekt war die } \\
\text { Grundlage für einen } \\
\text { neuen thematischen } \\
\text { Schwerpunkt }\end{array}$ & 42 & $33 \%$ & 21 & $35 \%$ & 63 & $33 \%$ \\
\hline & $\begin{array}{l}\text { Das Projekt betraf eher } \\
\text { einen Randbereich }\end{array}$ & 23 & $18 \%$ & 1 & $2 \%$ & 24 & $13 \%$ \\
\hline & Sonstiges & 2 & $2 \%$ & 1 & $2 \%$ & 3 & $2 \%$ \\
\hline & Gesamt & 129 & $100 \%$ & 60 & $100 \%$ & 189 & $100 \%$ \\
\hline
\end{tabular}

Quelle: KMU Forschung Austria

Dennoch darf man wohl schließen, dass das Matching von Unternehmen und Forschungsinstituten mit der relevanten Kompetenz bzw. Interesse im industrienahen Bereich bereits gut funktioniert.

Durchschnittlich knapp 2 Diplomarbeiten, 1,5 Dissertationen, und 3,1 ForschungsmitarbeiterInnen ab Post-Doc Niveau je Projektteilnahme

Für die Erfüllung der FuE-Tätigkeiten wurden von den 118 Forschungseinrichtungen 971 MitarbeiterInnen zur Bearbeitung der Aufgabenstellungen einbezogen. Mehr als die Hälfte davon war Forschungspersonal auf der Ebene von Post-docs, LabormitarbeiterInnen, DozentInnen, etc. (82\% der FE setzten MitarbeiterInnen dieser Kategorie ein). Des Weiteren waren 254 Diplomandlnnen (in 107 Projekten) und 204 Doktorandlnnen (in 111 Projekten) in die FuE-Tätigkeiten eingebunden. Die Projekte mit der höchsten MitarbeiterInnenzahl sind logischerweise bei den strukturellen Investitionen, z.B. bei dem Research Studios Austria, aber auch bei den FHs zu finden. Im Median sind je Projekt ein/e Diplomandln sowie ein/e Dissertantln, und zwei Forschungsmitarbeiterlnnen involviert. 
Tabelle 18 Verteilung der am Projekt beteiligten FuE-Mitarbeiterlnnen

\begin{tabular}{|l|c|c|}
\hline Projektmitarbeiterlnnen & Anzahl & Anteil \\
\hline DiplomandInnen & 254 & $26 \%$ \\
\hline Doktorandlnnen & 204 & $21 \%$ \\
\hline Anderes Forschungspersonal & 513 & $53 \%$ \\
\hline \hline Gesamt & 971 & $100 \%$ \\
\hline
\end{tabular}

Quelle: KMU Forschung Austria; $n=185$ Projektteilnahmen

Die Größe der involvierten Projektteams lag zwischen 19 und einer FuEMitarbeiterln. Für 58\% der Projekte wurden kleine Forschungsgruppen zwischen zwei und fünf MitarbeiterInnen eingesetzt (Vorjahr: 60\%).

\section{Abbildung 26 Größe der involvierten FuE-Mitarbeiterlnnenteams}

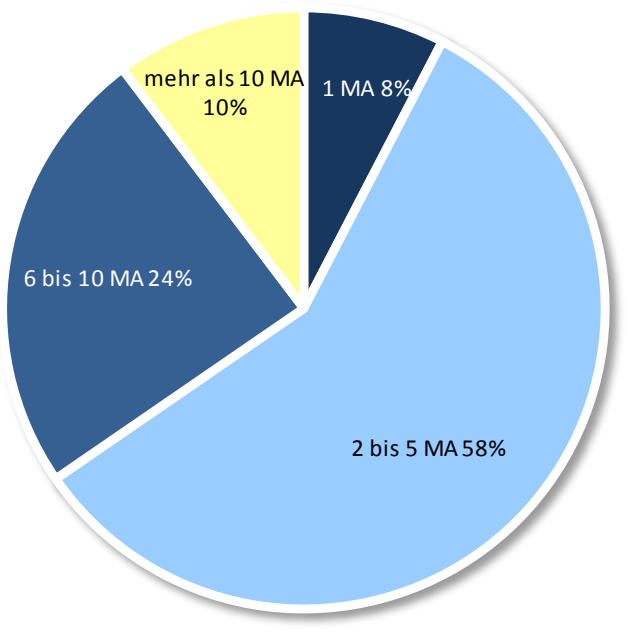

Quelle: KMU Forschung Austria; N=189 Projektteilnahmen 


\section{Hohe technische Erfolgsrate bei der Befragung der Forschungseinrichtungen bestätigt die langjährigen Ergebnisse der Unternehmensbefragung}

Knapp zwei Drittel der Forschungseinheiten konnten die eigenen Projektteile in vollem Umfang umsetzen. Nach Einschätzung der FE konnten in der Hälfte der Projekte auch die Ziele des Gesamtprojektes erreicht werden; in weiteren $44 \%$ „trifft [dies] ziemlich zu“. Dies darf wohl dahingehend interpretiert werden, dass die Projekte insofern erfolgreich waren, als die Resultate nutzbringend eingesetzt werden konnten, auch wenn nicht alle Projektziele erfüllt wurden. Zusammengenommen decken sich die rd. 90\% mit den Angaben der Unternehmen zur technischen Erfolgsrate der Projekte, und signalisiert die Plausibilität der Werte.

\section{Abbildung 27 Inhaltliche Projektzielerreichung}

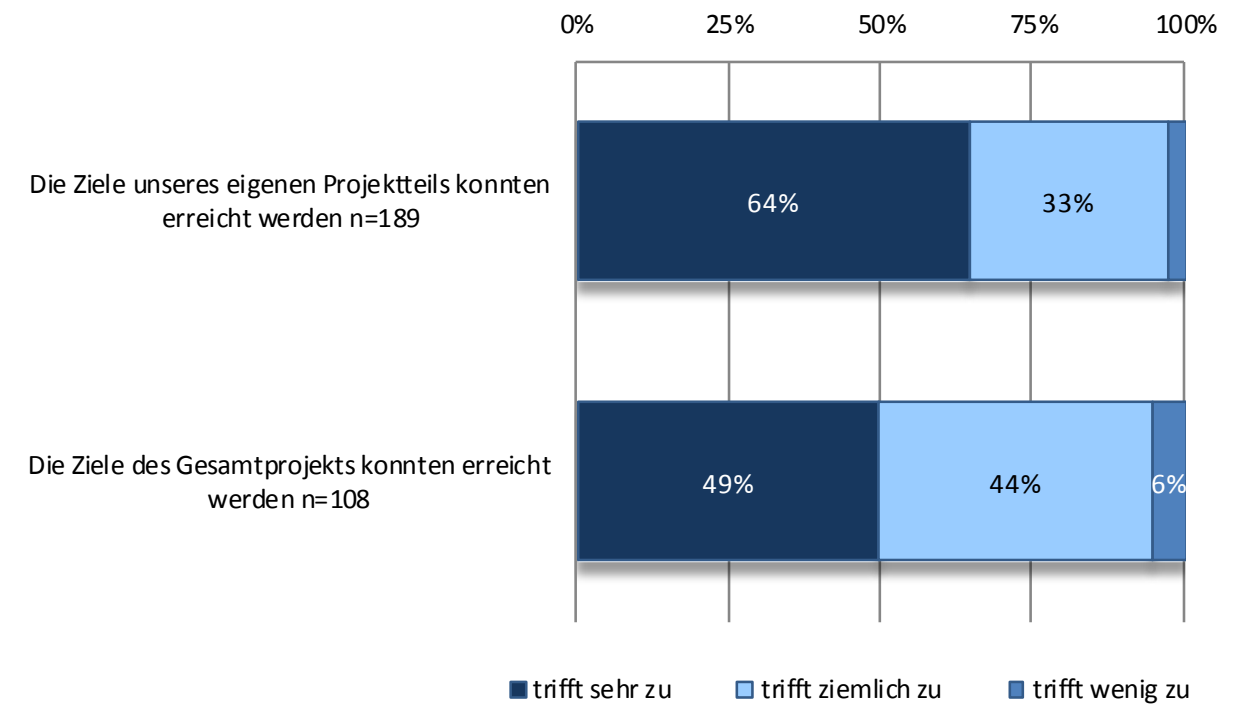

Quelle: KMU Forschung Austria; Zeile 2 bereinigt um Mehrfachnennungen durch Projektteilnehmer

\section{Bei den Projekten werden neue Kooperationen angestoßen, die eine relativ hohe Zufriedenheit auch mit neuen Partnern nach sich ziehen}

Bei den untersuchten Projekten handelt es sich nahezu ausschließlich um Kooperationsprojekte (94\%). Die vorherige Kooperationserfahrung mit einem wissenschaftlichen Partner beeinflusst die Zufriedenheit im Nachhinein nicht sehr. Bei ersteren ist die Zufriedenheit mit 48\% (sehr erfolgreich) nicht dramatisch höher als bei einer erstmaligen Zusammenarbeit $41 \%$. Das insgesamt doch gute Ergebnis wird statistisch etwas ins Negative verzerrt, da einige der Antwortenden nicht direkt mit den Projektpartnern interagierten, und aufgrund dessen diese Frage nicht beantworten konnten. Dafür ist der zeitliche Abstand zum Projektende von vier Jahren verantwortlich. 
Eine nicht zufriedenstellende FuE Kooperation begründen die FE meist mit fehlendem Engagement der Projektpartner (eher Unternehmen), geringe Forschungskapazitäten der Partner oder fehlende Folgeprojekte. Eine Auswertung nach Instrumenten ist hier erst mit Daten für mehrere Jahre zusammengenommen anzuraten.

Abbildung 28 Wie erfolgreich stufen Sie die FuE Kooperation im konkreten Projekt ein?

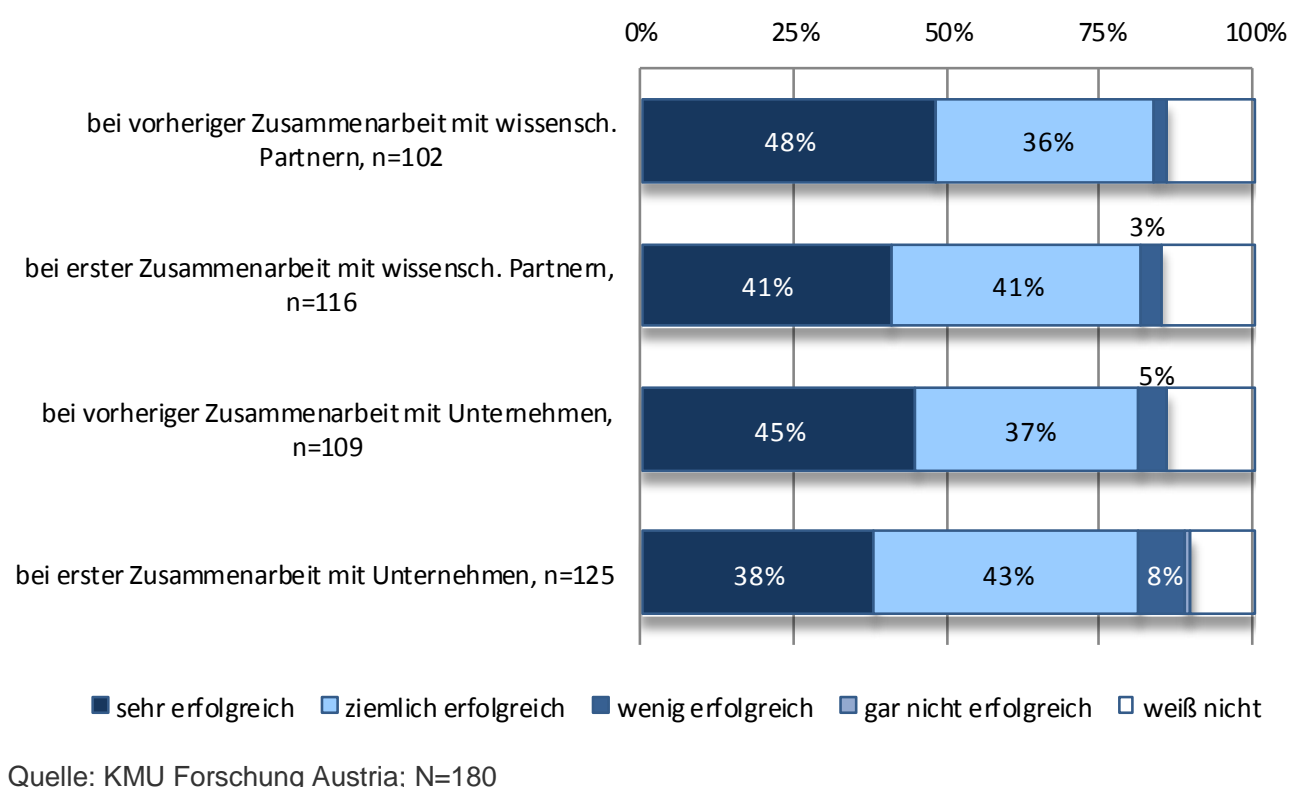

Quelle: KMU Forschung Austria; N=180

Im zweiten Teil der Fragestellung konnten die FE angeben, ob sie mit den jeweiligen Unternehmen bzw. wissenschaftlichen Partnern auch in Zukunft zusammenarbeiten wollen. Wenig überraschend stehen bereits längerfristig bestehende Kooperationen noch höher im Kurs (wissenschaftlicher Partner: 69\%, Unternehmen 58\% mit eindeutigem ,Ja'), aber auch eine erstmalige Kooperation (wissenschafticher Partner: $52 \%$, Unternehmen $54 \%$ ) hat relativ oft den Boden für eine mögliche weitere $\mathrm{Zu}$ sammenarbeit aufbereitet. 


\section{Effekte auf die Forschungseinheiten und Spillovers}

In 57\% aller 163 Forschungsprojekte (93 Projekte = 114 Projektteilnahmen) gab es eine Festlegung der Verwertungsrechte (in Form von Konsortialverträgen oder Absprachen).

Von diesen 114 Projektteilnehmern waren in 15\% der Fälle FE schlussendlich auch an den Schutzrechten beteiligt. Die FE sicherten sich in 17 Projektteilnahmen mit 25 Schutzrechten ihre Forschungsergebnisse entweder allein oder zusammen mit Projektpartnern ab. Bezogen auf alle 192 Projektteilnahmen bedeutet dies einen Anteil von 9\% (Vorjahr: 8\%). 15\% der Befragten konnten diese Frage jedoch nicht beantworten.

Aus der Unternehmensbefragung wissen wir, dass 24\% der Projektresultate zu Schutzrechtsanmeldungen führten. Letzteres ist ein erheblicher Anteil und unterstreicht, auch vor dem Hintergrund der fallweisen Interessenskonflikte hinsichtlich der wirtschaftlichen Umsetzung der Projektergebnisse, die von der FFG vorangetriebene Durchsetzung von Konsortialverträgen.

Tabelle 19 Gewerbliche Schutzrechte unter Teilnahme der Forschungseinheiten

\begin{tabular}{|l|c|c|c|c|}
\hline \multirow{2}{*}{ Art der Schutzrechte } & \multicolumn{2}{c|}{ Eigene Institution } & \multicolumn{2}{c|}{ Mit Projektpartner } \\
\cline { 2 - 5 } & Nennung & Anzahl & Nennung & Anzahl \\
\hline Technische: Patente & 5 & 7 & 10 & 14 \\
\hline $\begin{array}{l}\text { Technische: Gebrauchsmuster, Sorten- } \\
\text { oder Halbleiterschutz, etc. }\end{array}$ & - & - & 1 & 1 \\
\hline $\begin{array}{l}\text { Nicht-technische: Marken, eingetragene } \\
\text { Designs, etc. }\end{array}$ & 1 & 3 & - & - \\
\hline \hline Gesamt & 6 & 10 & 11 & 15 \\
\hline
\end{tabular}

Quelle: KMU Forschung Austria; N=192 


\section{Projektergebnisse benötigen einerseits noch weiterer Entwicklungen, und sind andererseits auch für weitere Anwendungsgebiete interessant}

Die folgende Fragestellung gibt Hinweise zum Stadium sowie dem Spektrum der Umsetzung von Projektergebnissen, soweit dies den FE bekannt ist. Der manchmal doch beträchtliche Anteil der Kategorie „weiß nicht“ beleuchtet, dass die FE nicht in jedem Fall in etwaige innerbetriebliche Prozesse eingeweiht sind. Insgesamt sind die Resultate beinahe ident zum Vorjahresergebnis.

Der vorwettbewerbliche Charakter einiger Projekte wird dadurch unterstrichen, dass rd. ein Drittel der Projektergebnisse noch Entwicklungsschritte benötigen, um anwendbar zu werden. Jedes zweite Projektergebnis wird für die potenzielle Nutzung in anderen Anwendungsgebieten als zumindest ziemlich relevant eingeschätzt. In einer offenen Fragestellung, in welchen anderen Anwendungsgebieten die Projektergebnisse genutzt werden könnten, werden 76 weitere Anwendungsgebiete angesprochen, von denen einige präzise formuliert werden und eine spezielle Branche adressieren, und andere sehr generischer Natur sind und deshalb ein sehr breites Anwendungsfeld in sehr unterschiedlichen Branchen versprechen. Es stellt sich die Frage, inwiefern eine Überführung in andere Anwendungsgebiete erleichtert werden kann. Hier könnten sich manche FE als zentrale Akteure anbieten. Die Transfermechanismen des FFG Portfolios sowie die Rolle der Ausgestaltung von Verwertungsrechten könnten daraufhin noch analysiert werden.

\section{Abbildung 29 Wie beurteilen Sie die Umsetzungs- und Nutzungsmöglichkeiten der} Projektergebnisse?

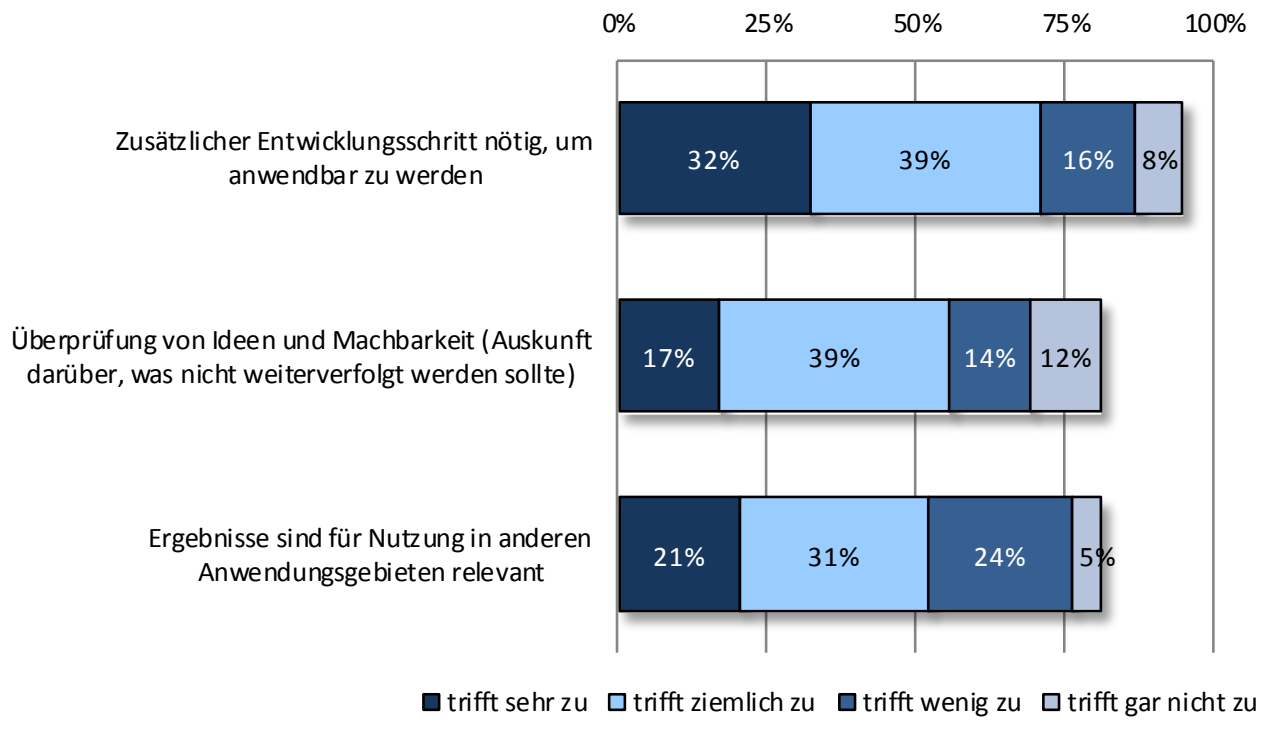

Quelle: KMU Forschung Austria, N=187 Projektteilnahmen; die fehlenden Antwortkategorien auf $100 \%$ verteilen sich auf ,weiß nicht' und ,nicht relevant". 


\section{Neue Kontakte sowie die Heranführung von wissenschaftlichem Nachwuchs als wichtige Wirkungskategorien}

Beinahe drei Viertel der Projektteilnahmen führten zu neuen Kontakten/ Kooperationen für die FE. Diese wurden mit 345 neuen Kontakten quantifiziert (1,8/Projekt). In rd. der Hälfte der Fälle wurden nach Projektende insgesamt 217 der beteiligten DiplomandInnen/DissertantInnen an den FE weiterbeschäftigt. Ebenso bei $51 \%$ führte das Projekt zu insgesamt 141 weiterführenden Diplomarbeiten oder Dissertationen an den FE.

Der Wechsel von FuE-Personal (inkl. Diplomandlnnen oder Dissertantlnnen) zu Unternehmenspartnern des Projekts erfolgte bei 16\% der Projektbeteiligungen und bei weiteren $7 \% \mathrm{zu}$ wissenschaftlichen und anderen Kooperationspartnern. Alle Prozentwerte sind nahezu ident mit den Ergebnissen des Vorjahres.

Abbildung 30 Welche der folgenden Effekte ergaben sich aufgrund des FFG-Projekts?

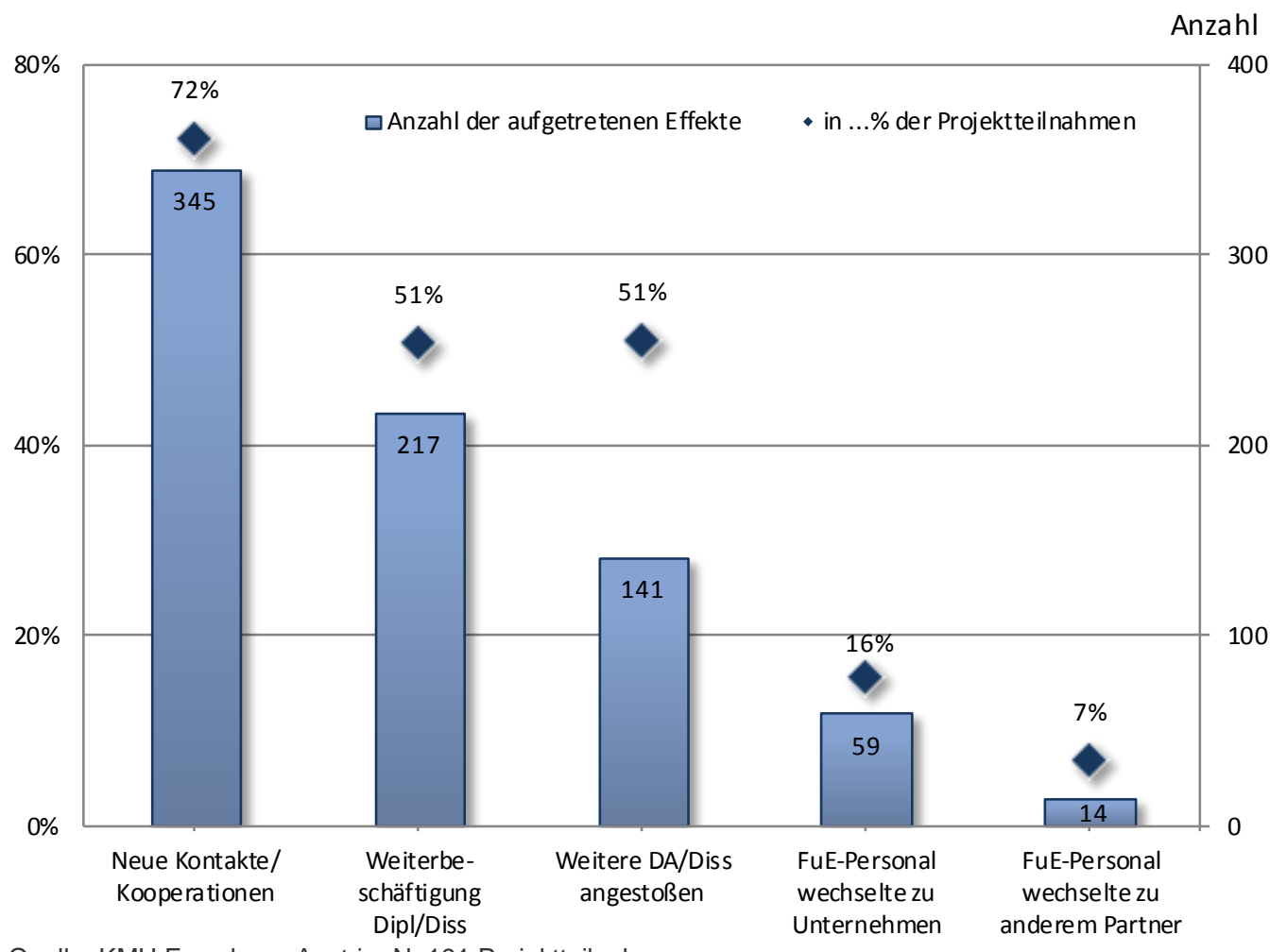

Quelle: KMU Forschung Austria; N=191 Projektteilnahmen 


\section{Folgeprojekte werden zu rd. $42 \%$ wieder von der FFG unterstützt}

Im Rahmen von 109 Projektbeteiligungen (57\% aller Projektbeteiligungen) sind bislang mindestens 246 Folgeprojekte entstanden (einige Teilnehmer konnten die Zahl der entstandenen Projekte nicht konkretisieren). Darüber hinaus wurde von den befragten Forschungseinrichtungen angegeben, wie die Folgeprojekte finanziert wurden und welche Partner involviert waren. Folgeprojekte wurden in 35 Fällen mit demselben Konsortium umgesetzt, weitere 109 mit Teilen des Konsortiums und 108 mit neuen Partnern. Diese Nennungen summieren sich auf insgesamt 286 fortgesetzte FuE-Aktivitäten in verschiedenen Konsortien.

Rd. 43\% der Projektteilnahmen werden wieder durch die FFG unterstützt, der nächsthöhere Anteil sind direkt durch Unternehmen (u.a. Institutionen) unterstützte Projekte (21\%); die 41 Folgeprojekte auf EU Ebene entsprechen rd. 14\% der 286 Nennungen.

Auf die ursprünglichen 192 Projektbeteiligungen bezogen lautet die Verteilung der Finanzierung der Folgeprojekte folgendermaßen: 64\% (FFG), 31\% (Unternehmen), $21 \%$ (EU), sowie 20\% (andere Förderprogramme).Immerhin 14\% der Folgeprojekte sind eigenfinanziert, da das Eigeninteresse der Forschungseinrichtung groß genug zu sein schien, um Verwertungspotentiale heben zu wollen.

Tabelle 20 Anzahl und Art der Folgeprojekte und deren Finanzierung

\begin{tabular}{|l|c|c|c|c|c||c|c|}
\hline & $\begin{array}{c}\text { Selbes } \\
\text { Konsor- } \\
\text { tium } \\
\text { Konsor- } \\
\text { tiums }\end{array}$ & $\begin{array}{c}\text { Teile } \\
\text { Partner }\end{array}$ & Alleine & Gesamt & $\begin{array}{c}\text { Anteil } \\
\text { an } \\
\text { Folge- } \\
\text { projek- } \\
\text { ten }\end{array}$ & $\begin{array}{c}\text { Anteil } \\
\text { an } \\
\text { Pro- } \\
\text { jektbe- } \\
\text { teili- } \\
\text { gungen }\end{array}$ \\
\hline $\begin{array}{l}\text { FFG Folge- } \\
\text { projekte }\end{array}$ & 18 & 52 & 48 & 4 & 122 & $43 \%$ & $64 \%$ \\
\hline $\begin{array}{l}\text { EU Folge- } \\
\text { projekte }\end{array}$ & 2 & 18 & 21 & 0 & 41 & $14 \%$ & $21 \%$ \\
\hline $\begin{array}{l}\text { Andere Förder- } \\
\text { programme }\end{array}$ & 4 & 15 & 11 & 8 & 38 & $13 \%$ & $20 \%$ \\
\hline $\begin{array}{l}\text { Direkt durch } \\
\text { Unternehmen / } \\
\text { Institutionen } \\
\text { finanziert }\end{array}$ & 6 & 18 & 24 & 11 & 59 & $21 \%$ & $31 \%$ \\
\hline Eigenfinanziert & 5 & 6 & 4 & 11 & 26 & $9 \%$ & $14 \%$ \\
\hline \hline Gesamt & 35 & 109 & 108 & 34 & 286 & $100 \%$ & $149 \%$ \\
\hline
\end{tabular}

Quelle: KMU Forschung Austria; N=192 Projektteilnahmen 


\section{Disseminationsaktivitäten erfolgen primär mit Beteiligung der Projektpartner}

Die mit rd. 79\% am häufigsten gewählte Art der Dissemination der Projektergebnisse war zum einen Publikationen in akademisch referierten Journalen und Konferenzen. Der überwiegende Teil davon erfolgte zusammen mit den Projektpartnern, was auf eine relativ enge Kooperation während des Forschungsprozesses schließen lässt.

Weitere zwei Drittel (69\%) präsentierten die Forschungsergebnisse im Rahmen von akademischen Konferenzen, ohne dass damit eine referierte Publikation verbunden ist. Die Publikation in praxisorientierten Fachzeitschriften passierte in rd. der Hälfte der Projekte (57\%), hier sind die Projektpartner noch stärker eingebunden. Andersherum formuliert werden dieser Verbreitungsmedien aber schon in $44 \%$ der Projekte nicht genutzt, da Unternehmen dies wohl kaum allein umsetzen.

Aufgrund der institutionellen Anreizmechanismen legen Universitäten und Kompetenzzentren mehr Wert auf referierte Journalartikel sowie Konferenzpräsentationen als außeruniversitäre FE.

Abbildung 31 Welche der folgenden Aktivitäten zum Transfer der Projektergebnisse wurden von Ihnen gesetzt?

Publikationen in akademischen Journalen und Konferenzen (referiert)

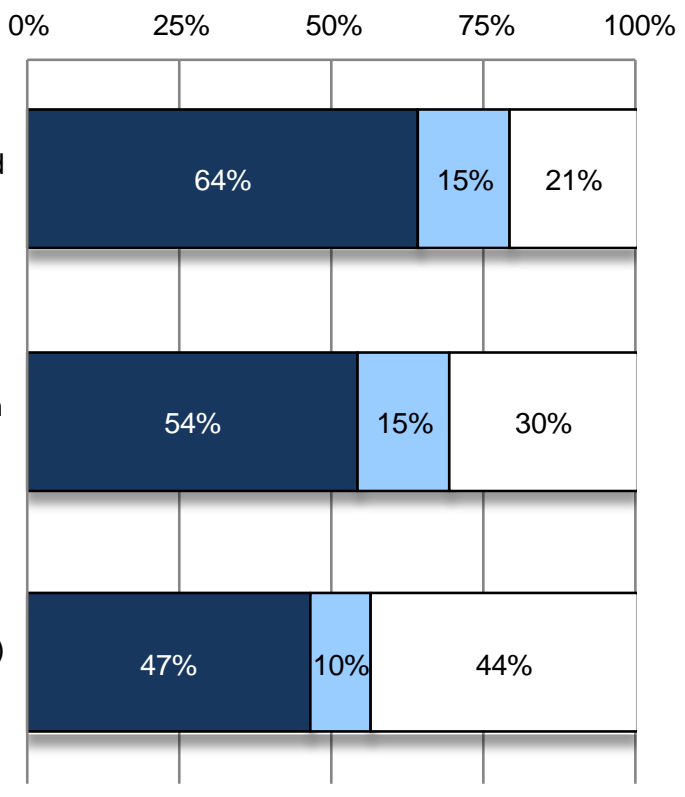

$\square$ Ja, mit Beteiligung der Projektpartner

$\square \mathrm{Ja}$, ohne Beteiligung der Projektpartner $\quad \square$ nein

Quelle: KMU Forschung Austria; N=191 Projektteilnahmen 
Die folgende Abbildung quantifiziert die Transferaktivitäten der FE sowie möglich (35 TeilnehmerInnen konnte die Zahl der Publikationen nicht spezifizieren). In Summe belaufen sich die Disseminationstätigkeiten auf 958 gesetzte Maßnahmen (durchschnittlich 5,0 je Projektteilnahme). Davon sind 255 referierte Publikationen mit, und 179 Publikationen ohne Kooperationspartner. Diese 493 Publikationen verteilen sich auf 104 Projektteilnahmen.

\section{Abbildung 32 Häufigkeiten der Disseminationsergebnisse}

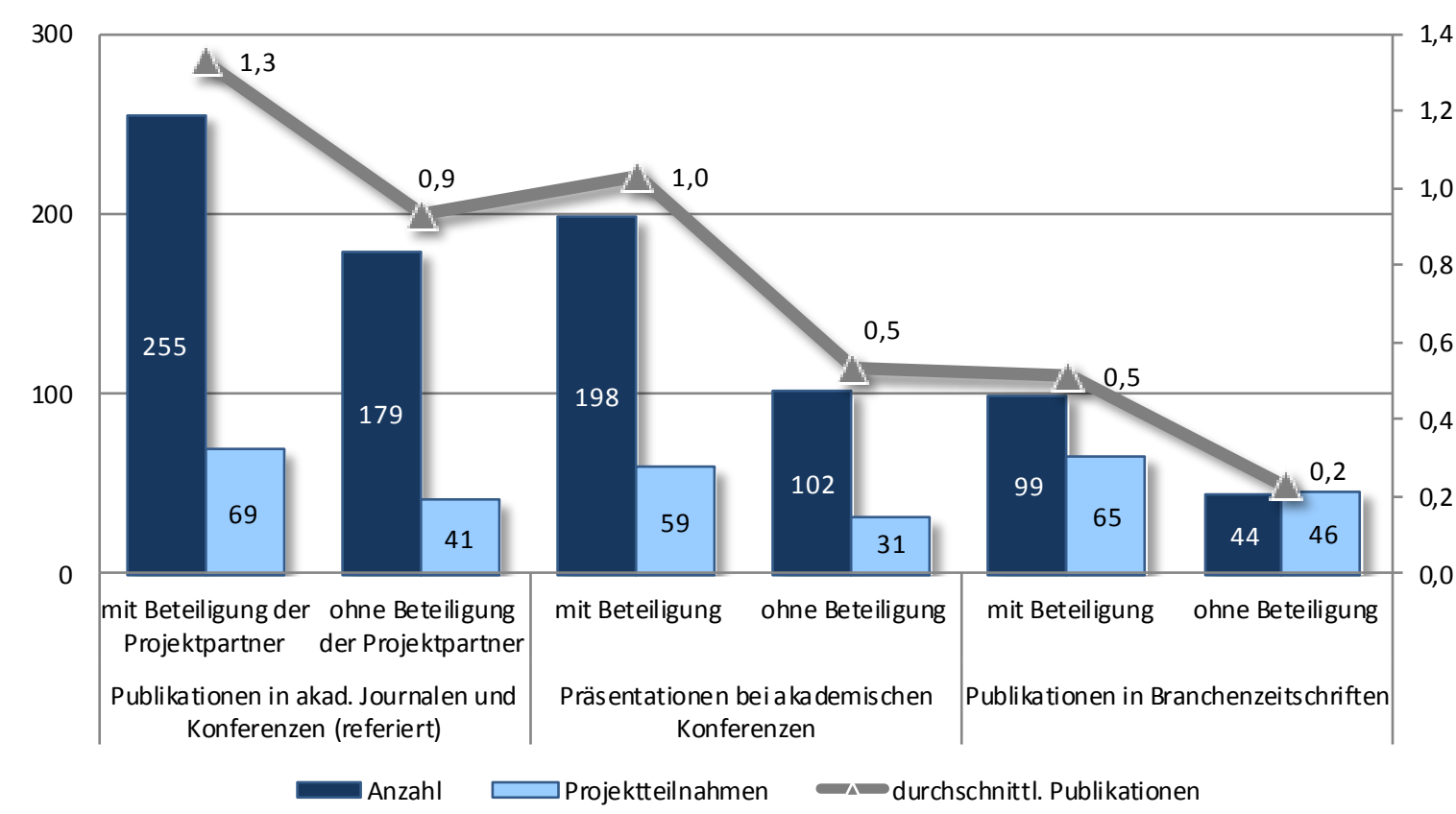

Quelle: KMU Forschung Austria; N=192 Projektteilnahmen (Dissemination mit Beteiligung von Projektpartnern bereinigt um Mehrfachnennungen) 


\section{Über Publikationen hinausgehend, noch weitere fünf Disseminationsaktivitä- ten je Projektbeteiligung}

Abgesehen von diesen „klassischen“ Wegen der Ergebnisverbreitung haben die Befragten FE auch weitere Aktivitäten durchgeführt. Etwa die Präsentation bei Veranstaltungen, oder die Zielgruppe war bereits in die Projektdurchführung eingebunden, bzw. die Zielgruppe des Projektes wurde bei der Umsetzung unterstützt. Auch wurden die Ergebnisse auf der Homepage der FE dargestellt bzw. wurden auch Forschungsberichte von den FE weitergegeben.

Den FE wurde die Möglichkeit gegeben die Zahl der Tagungen, Messen, Workshops, etc. zu quantifizieren, auf welchen sie die Ergebnisse präsentiert haben. Durch 136 Projektbeteiligungen wurden 678-mal die erzielten Resultate an ein Fachpublikum weitergegeben.

\section{Abbildung 33 Welche weiteren Transferaktivitäten führten Sie durch?}

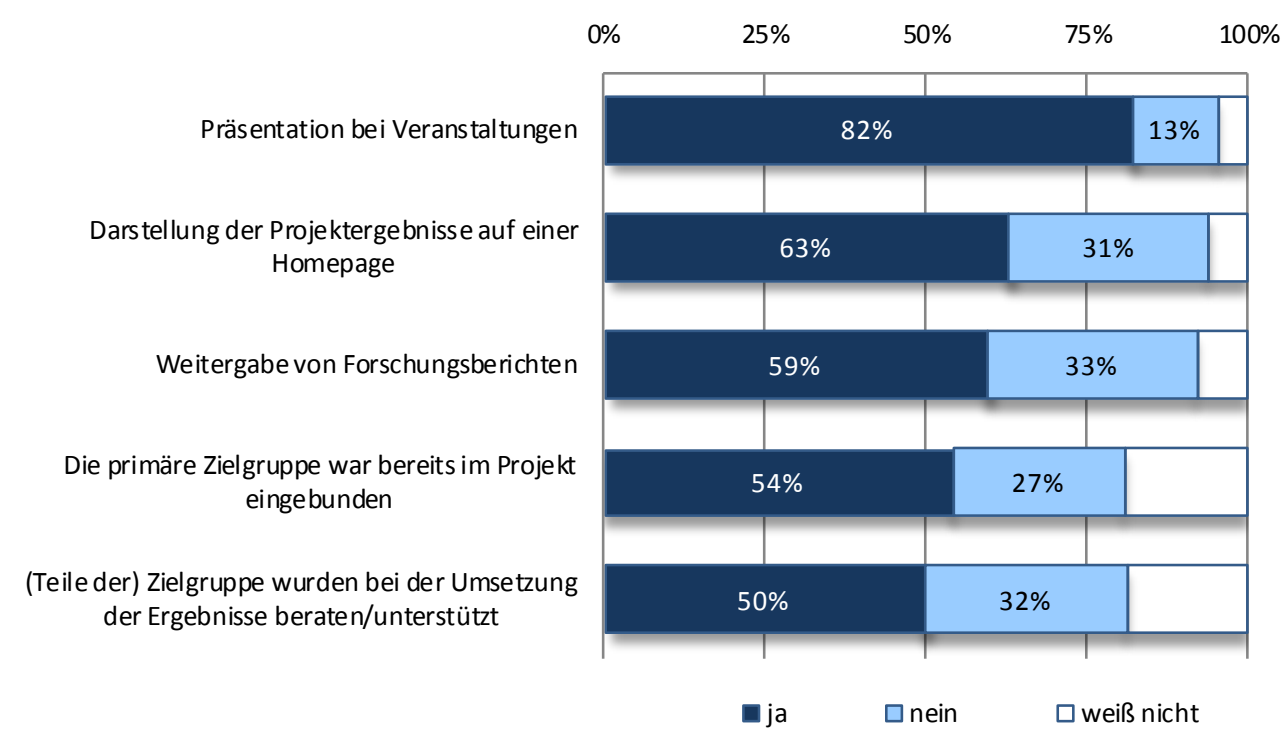

Quelle: KMU Forschung Austria; N=189 Projektteilnahmen 


\section{Wirkungen der Projekte primär durch erweiterte Expertise, Reputation und Netzwerkeffekte}

Über 90\% der Forschungseinheiten gestanden dem Projekt zu, die Expertise des Instituts erweitert zu haben. Rund vier Fünftel der Forschungsvorhaben führten zu einer erhöhten Attraktivität als Kooperationspartner und zur erhöhten Sichtbarkeit der Forschungseinrichtungen in der Zielgruppe. Auch die Erweiterung des Forschungsnetzwerks bzw. die Weiterverfolgung von anwendungsorientierten Fragen durch das Projekt wurden häufig als sehr zutreffend beurteilt. Neue Lehrinhalte sind natürlich nur für lehrende Forschungseinheiten relevant; hier ergeben sich jedoch deutliche Wechselwirkungen zwischen Lehre und Forschung.

Abbildung 34 Wie schätzen Sie die Wirkung des Projekts auf Ihr Institut ein?

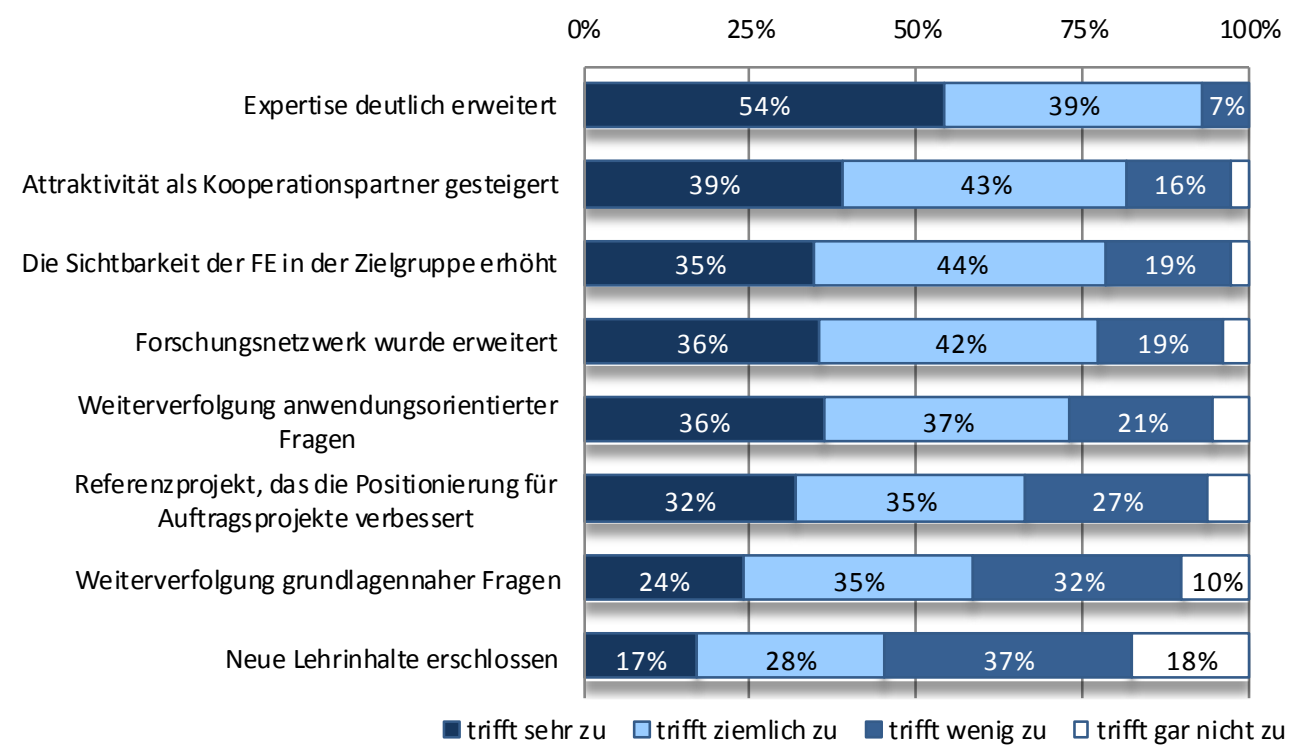

Quelle: KMU Forschung Austria; N=191 Projektteilnahmen

Abschließend wurden noch Fragen zum strukturellen Hintergrund der Forschungseinheiten gestellt, um deren Nähe zur industriellen Basis näherungsweise auszuleuchten. 
Knapp die Hälfte der FE ist häufig in der privaten, direkten Auftragsforschung aktiv; etwa ein Drittel auch häufig in beratender Funktion

Wie auch im letzten Jahr zeigen sich bei der Durchführung von privat beauftragten Projekten Unterschiede bei den verschiedenen Organisationstypen. Während rd. $65 \%$ der untersuchten außeruniversitären FE angeben (ähnlich auch K-Zentren), häufig private Auftragsforschung durchzuführen, sind es $44 \%$ bei den Universitätsinstituten (ähnlich auch FH-Institute).

Beratungsleistungen stellen für die Mehrheit der Forschungseinheiten eher die Ausnahme dar. Hier wurde nur mehr von rd. 30\% der FE angegeben, dass häufig direkte Beratungen für Unternehmen und Institutionen durchgeführt werden. Der Anteil häufig beratender AUF-Institute liegt bei rd. 50\%, jener der universitären FE bei rd. $25 \%$ und jener der $\mathrm{FH}-$ Institute bei $17 \%$.

Abbildung 35 Wie häufig führt Ihr Institut private Auftragsforschung bzw. Beratungsleistungen direkt für Unternehmen oder Institutionen durch?

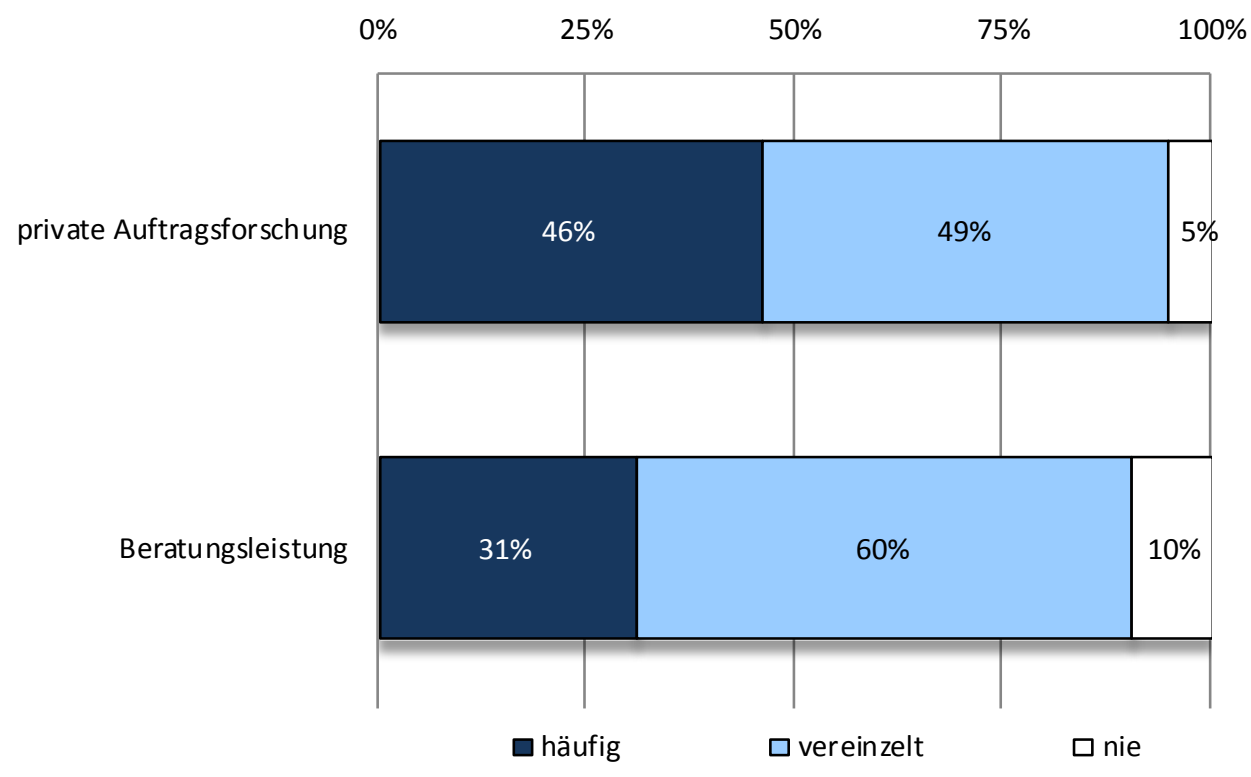

Quelle: KMU Forschung Austria; N=118 Forschungseinheiten 


\section{Anhang}

\section{Berechnungen}

\section{Beschäftigungseffekte}

zusätzliche Mitarbeiterlnnen

+ gesicherte Arbeitsplätze

- $\quad$ freigesetzte MitarbeiterInnen

Direkter Beschäftigungseffekt

\section{Multiplikator}

$$
\text { Fördermultiplikator M3 }=\frac{\text { Lizenzerlöse }+ \text { Zusatzumsätze }}{\text { Barwert der Förderungen }}
$$

\section{Tabellen}

Tabelle 21 KMU-Definition der Europäische Kommission

\begin{tabular}{|c|c|c|c|c|c|}
\hline Unternehmenskategorie & Mitarbeiterlnnen & \multirow{4}{*}{ 号 } & Umsatz & & ilanzsumme \\
\hline Kleinstunternehmen & $<10 \mathrm{VZÄ}$ & & $\leq 2$ Mio. $€$ & \multirow{3}{*}{$\begin{array}{l}\stackrel{⿰}{山} \\
\text { ○ }\end{array}$} & $\leq 2$ Mio. $€$ \\
\hline Kleinunternehmen & $<50$ VZÄ & & $\leq 10$ Mio. $€$ & & $\leq 10$ Mio. $€$ \\
\hline Mittlere Unternehmen & $<250$ VZÄ & & $\leq 50$ Mio. $€$ & & $\leq 43$ Mio. $€$ \\
\hline Großunternehmen & \multicolumn{5}{|c|}{ Abweichende Werte bzw. Eigentümerverhältnisse } \\
\hline
\end{tabular}

Quelle: Europäische Kommission

Diese Grenzwerte dürfen auch gemeinsam mit „Partnerunternehmen“ bzw. „verbundenen Unternehmen" nicht überschritten werden, die allein oder gemeinsam mit einem oder mehreren verbundenen Unternehmen 25\% oder mehr des Kapitals oder der Stimmrechte eines anderen Unternehmens halten. 


\section{Non-Response Analyse Unternehmensbefragung}

Hier werden die Projektteilnahmen näher analysiert, die einen Fragebogen zwar erhalten, aber nicht ausgefüllt haben. Entsprechend der Differenz von versendeten und eingegangenen Fragbögen (siehe Tabelle 1) können 185 Projektteilnahmen durch 154 Unternehmen als nicht beantwortet gewertet werden.

Nach Instrumenten überwiegen die Kooperationsprojekte (92 bzw. 50\%), ein weiteres Drittel entfällt auf die Einzelprojekte (63, bzw. 24\%) und rd. 9\% (16) sind Projekte der Innovationsnetzwerke. In Hinblick auf die Größenklassen der nicht-teilnehmenden Unternehmen lassen sich keine Unterschiede zu den antwortenden Fördernehmern feststellen. 47\% der Projektteilnahmen sind auf KU zurückzuführen, 14\% auf MU und $39 \%$ GU.

Auch in Bezug auf die Projektgrößen und Fördersummen sind die Unterschiede gering. Die Gesamtkosten der relevanten Projekte belaufen sich auf rd. $€ 77,3$ Mio. bei einem Förderbarwert von $€ 26,5$ Mio. Dies entspricht wiederum einem Anteil von etwa einem Drittel der Gesamtsummen. Die zugehörigen Durchschnittswerte liegen bei rd. $€ 417.790$ und $€ 143.250$ und unterscheiden sich konsequenterweise nur marginal von den teilnehmenden Unternehmen.

Die untenstehende Tabelle ist mit jener in Kapitel 4 zu vergleichen. Es fällt einzig auf, dass sich nach Größenklassen Unternehmen mit leicht höheren Projektvolumina an der Befragung beteiligten.

Tabelle 22 Durchschnittliche Gesamtkosten und Förderbarwerte nichtantwortender Unternehmen

\begin{tabular}{|c|c|c|c|c|}
\hline \multirow{2}{*}{} & \multicolumn{2}{|c|}{ Gesamtkosten } & \multicolumn{2}{c|}{ Förderbarwert } \\
\cline { 2 - 5 } & Mittelwert & Median & Mittelwert & Median \\
\hline Einzelprojekt & 827.484 & 340.000 & 229.295 & 119.973 \\
\hline Koop.Projekt & 229.608 & 106.455 & 113.514 & 62.975 \\
\hline Wiss.Transfer & 49.363 & 50.750 & 30.553 & 28.950 \\
\hline Markteinführung & 337.435 & 246.409 & 84.359 & 61.602 \\
\hline Inno.Netzwerk & 101.058 & 66.880 & 53.876 & 37.049 \\
\hline \hline KU & 188.247 & 115.059 & 94.469 & 63.670 \\
\hline MU & 234.461 & 145.700 & 92.069 & 63.000 \\
\hline GU & 754.149 & 223.462 & 218.916 & 75.000 \\
\hline
\end{tabular}

Quelle: KMU Forschung Austria, N=185

52\% der nicht-beantworteten Projektteilnahmen sind Bestandskunden der FFG zuzurechnen. Dementsprechend gibt es auch hier lediglich marginale Unterschiede zu den Befragungsteilnehmern. 


\section{Non-Response Analyse Forschungseinrichtungen}

Die nicht-beantworteten Projektteilnahmen der FE lassen sich von Tabelle 15 ableiten. Insgesamt haben 211 (52\%) der 403 Befragten nicht geantwortet, wobei diese sich auf 170 Projekte aufteilen. Die dominierenden Programme sind dabei auf Grund der hohen Projektzahlen: Neue Energien 2020 (25\%) und IV2Splus (19\%) bzw. das Förderinstrument der Kooperationsprojekte im Allgemeinen (74\%, BRIDGE 12\%, und Einzelprojekte 7\%). Dabei verteilen sich die Projektteilnahmen auf 139 unterschiedliche Forschungseinheiten.

Fast die Hälfte der ausstehenden Fragebögen können Universitätsinstituten zugerechnet werden (48\%) und weitere $35 \%$ außeruniversitären Forschungseinrichtungen. FHs sind 12 Projektteilnahmen schuldig geblieben (6\%) und Kompetenzzentren haben in elf Fällen nicht geantwortet (6\%).

Die Gesamtkosten der relevanten Projekte belaufen sich auf rd. $€ 43,1$ Mio. bei einem Förderbarwert von $€ 25,7$ Mio. Die zugehörigen Durchschnittswerte liegen bei rd. $€ 204.400$ und $€$ 121.800. In Bezug auf die einzelnen Instrumente und Größenklassen zeigen sich nur Unterschiede bei den Kategorien mit geringen Fallzahlen.

Diese Ergebnisse entsprechen den antwortenden FE erstaunlich gut, und es kann von einem repräsentativen Ausschnitt der FFG geförderten Forschungseinheiten ausgegangen werden. Dies wird auch dadurch unterstützt, als die Ergebnisse dieses Jahres mit jenen des Vorjahres in sehr hohem Ausmaß übereinstimmen. 
Tabelle 23 Frage 7: Wurde das Projektziel aus technischer / wirtschaftlicher Sicht erreicht? Im Bundesländervergleich

\begin{tabular}{|c|c|c|c|c|c|c|c|c|c|c|c|c|c|c|c|c|c|c|c|c|}
\hline & \multicolumn{2}{|c|}{$\begin{array}{l}\text { Gesamt- } \\
\text { summe }\end{array}$} & \multicolumn{2}{|c|}{ Burgenland } & \multicolumn{2}{|c|}{ Kärnten } & \multicolumn{2}{|c|}{$\begin{array}{l}\text { Niederöster- } \\
\text { reich }\end{array}$} & \multicolumn{2}{|c|}{$\begin{array}{l}\text { Oberöster- } \\
\text { reich }\end{array}$} & \multicolumn{2}{|c|}{ Salzburg } & \multicolumn{2}{|c|}{ Steiermark } & \multicolumn{2}{|c|}{ Tirol } & \multicolumn{2}{|c|}{ Vorarlberg } & \multicolumn{2}{|c|}{ Wien } \\
\hline & abs. & in $\%$ & abs. & in $\%$ & abs. & in $\%$ & abs. & in $\%$ & abs. & in $\%$ & abs. & in $\%$ & abs. & in $\%$ & abs. & in $\%$ & abs. & in $\%$ & abs. & in $\%$ \\
\hline Projekte insgesamt & 548 & 100,0 & 5 & 0,9 & 25 & 4,6 & 66 & 12,0 & 108 & 19,7 & 26 & 4,7 & 107 & 19,5 & 28 & 5,1 & 22 & 4,0 & 161 & 29,4 \\
\hline Gesamtzuschuss*) & 75,5 & 100,0 & 0,8 & 1,0 & 4,3 & 5,6 & 7,7 & 10,3 & 13,8 & 18,3 & 2,5 & 3,4 & 16,7 & 22,1 & 4,9 & 6,5 & 4,4 & 5,8 & 20,4 & 27,1 \\
\hline Darlehen*) & 30,2 & 100,0 & 0,1 & 0,2 & 0,8 & 2,5 & 3,5 & 11,7 & 5,6 & 18,5 & 1,5 & 4,8 & 7,4 & 24,5 & 1,3 & 4,2 & 2,3 & 7,6 & 7,8 & 26,0 \\
\hline technischer Sicht & 480 & 87,6 & 5 & 1,0 & 22 & 4,6 & 61 & 12,7 & 92 & 19,2 & 22 & 4,6 & 91 & 19,0 & 21 & 4,4 & 21 & 4,4 & 145 & 30,2 \\
\hline Gesamtzuschuss*) & 65,3 & 86,5 & 0,8 & 1,2 & 3,8 & 5,9 & 7,2 & 11,0 & 11,2 & 17,1 & 2,3 & 3,6 & 15,1 & 23,1 & 3,0 & 4,5 & 4,3 & 6,6 & 17,7 & 27,0 \\
\hline Darlehen*) & 26,5 & 87,8 & 0,1 & 0,2 & 0,8 & 2,9 & 2,9 & 10,8 & 4,7 & 17,7 & 1,1 & 4,3 & 7,1 & 26,6 & 0,9 & 3,2 & 2,3 & 8,5 & 6,9 & 25,8 \\
\hline $\begin{array}{l}\text { wirtschaftlicher } \\
\text { Sicht }\end{array}$ & 293 & 53,5 & 3 & 1,0 & 10 & 3,4 & 35 & 11,9 & 57 & 19,5 & 12 & 4,1 & 66 & 22,5 & 12 & 4,1 & 13 & 4,4 & 85 & 29,0 \\
\hline Gesamtzuschuss*) & 45,3 & 59,9 & 0,7 & 1,5 & 1,5 & 3,3 & 5,2 & 11,5 & 8,5 & 18,8 & 1,2 & 2,6 & 12,4 & 27,4 & 1,8 & 3,9 & 2,5 & 5,5 & 11,6 & 25,5 \\
\hline Darlehen*) & 20,1 & 66,4 & 0,0 & 0,0 & 0,5 & 2,6 & 2,9 & 14,4 & 3,6 & 17,8 & 0,9 & 4,4 & 5,4 & 26,8 & 0,6 & 3,1 & 1,4 & 7,1 & 4,8 & 23,7 \\
\hline
\end{tabular}

*) Beträge in Millionen Euro 
Frage 10: Werden die Projektergebnisse im Unternehmen wirtschaftlich verwertet? Im Bundesländervergleich

\begin{tabular}{|c|c|c|c|c|c|c|c|c|c|c|c|c|c|c|c|c|c|c|c|c|}
\hline & \multicolumn{2}{|c|}{$\begin{array}{l}\text { Gesamt- } \\
\text { summe }\end{array}$} & \multicolumn{2}{|c|}{ Burgenland } & \multicolumn{2}{|c|}{ Kärnten } & \multicolumn{2}{|c|}{$\begin{array}{l}\text { Niederöster- } \\
\text { reich }\end{array}$} & \multicolumn{2}{|c|}{$\begin{array}{l}\text { Oberöster- } \\
\text { reich }\end{array}$} & \multicolumn{2}{|c|}{ Salzburg } & \multicolumn{2}{|c|}{ Steiermark } & \multicolumn{2}{|c|}{ Tirol } & \multicolumn{2}{|c|}{ Vorarlberg } & \multicolumn{2}{|c|}{ Wien } \\
\hline & abs. & in $\%$ & abs. & in $\%$ & abs. & in $\%$ & abs. & in \% & abs. & in $\%$ & abs. & in $\%$ & abs. & in \% & abs. & in \% & abs. & in $\%$ & abs. & in \% \\
\hline Projekte insgesamt & 548 & 100,0 & 5 & 0,9 & 25 & 4,6 & 66 & 12,0 & 108 & 19,7 & 26 & 4,7 & 107 & 19,5 & 28 & 5,1 & 22 & 4,0 & 161 & 29,4 \\
\hline Gesamtzuschuss*) & 75,5 & 100,0 & 0,8 & 1,0 & 4,3 & 5,6 & 7,7 & 10,3 & 13,8 & 18,3 & 2,5 & 3,4 & 16,7 & 22,1 & 4,9 & 6,5 & 4,4 & 5,8 & 20,4 & 27,1 \\
\hline Darlehen*) & 30,2 & 100,0 & 0,1 & 0,2 & 0,8 & 2,5 & 3,5 & 11,7 & 5,6 & 18,5 & 1,5 & 4,8 & 7,4 & 24,5 & 1,3 & 4,2 & 2,3 & 7,6 & 7,8 & 26,0 \\
\hline $\begin{array}{l}\text { werden wirtsch. } \\
\text { verwertet }\end{array}$ & 266 & 48,5 & 2 & 0,8 & 14 & 5,3 & 36 & 13,5 & 54 & 20,3 & 10 & 3,8 & 59 & 22,2 & 13 & 4,9 & 13 & 4,9 & 65 & 24,4 \\
\hline Gesamtzuschuss*) & 43,6 & 57,7 & 0,5 & 1,2 & 1,9 & 4,3 & 5,0 & 11,6 & 8,0 & 18,3 & 1,3 & 3,0 & 11,1 & 25,6 & 3,1 & 7,1 & 3,0 & 6,8 & 9,6 & 22,1 \\
\hline Darlehen*) & 20,2 & 66,8 & 0,0 & 0,0 & 0,8 & 3,8 & 3,0 & 15,0 & 3,7 & 18,4 & 1,1 & 5,2 & 5,7 & 28,4 & 0,7 & 3,5 & 0,8 & 3,7 & 4,4 & 21,8 \\
\hline $\begin{array}{l}\text { in Zukunft wirtsch. } \\
\text { verwertet }\end{array}$ & 95 & 17,3 & 2 & 2,1 & 6 & 6,3 & 16 & 16,8 & 14 & 14,7 & 7 & 7,4 & 17 & 17,9 & 7 & 7,4 & 7 & 7,4 & 19 & 20,0 \\
\hline Gesamtzuschuss*) & 14,0 & 18,6 & 0,2 & 1,4 & 1,8 & 12,9 & 1,8 & 12,8 & 1,8 & 13,0 & 0,3 & 2,4 & 2,0 & 14,0 & 0,5 & 3,9 & 1,3 & 9,5 & 4,2 & 30,1 \\
\hline Darlehen ${ }^{\star}$ ) & 5,1 & 16,9 & 0,1 & 1,0 & 0,0 & 0,0 & 0,3 & 5,9 & 0,6 & 11,0 & 0,1 & 2,8 & 0,4 & 8,6 & 0,3 & 6,5 & 1,6 & 30,4 & 1,7 & 33,9 \\
\hline Erkenntniserweiterung & 132 & 24,1 & 1 & 0,8 & 2 & 1,5 & 10 & 7,6 & 27 & 20,5 & 7 & 5,3 & 26 & 19,7 & 5 & 3,8 & 1 & 0,8 & 53 & 40,2 \\
\hline Gesamtzuschuss*) & 13,4 & 17,8 & 0,0 & 0,3 & 0,1 & 0,9 & 0,6 & 4,6 & 2,5 & 18,8 & 0,7 & 5,4 & 3,4 & 25,6 & 1,0 & 7,8 & 0,0 & 0,3 & 4,9 & 36,3 \\
\hline Darlehen*) & 4,2 & 13,7 & 0,0 & 0,0 & 0,0 & 0,0 & 0,2 & 3,9 & 0,9 & 22,1 & 0,2 & 5,5 & 1,2 & 29,6 & 0,0 & 0,9 & 0,0 & 0,0 & 1,6 & 38,0 \\
\hline keine Verwertung & 23 & 4,2 & 0 & 0,0 & 1 & 4,3 & 3 & 13,0 & 7 & 30,4 & 2 & 8,7 & 1 & 4,3 & 2 & 8,7 & 1 & 4,3 & 6 & 26,1 \\
\hline Gesamtzuschuss*) & 2,6 & 3,4 & 0,0 & 0,0 & 0,3 & 9,8 & 0,1 & 4,2 & 1,1 & 42,6 & 0,2 & 7,1 & 0,0 & 1,0 & 0,1 & 4,9 & 0,0 & 1,4 & 0,8 & 29,0 \\
\hline Darlehen*) & 0,6 & 0,7 & 0,0 & 0,0 & 0,0 & 0,0 & 0,0 & 2,6 & 0,4 & 70,9 & 0,0 & 4,0 & 0,0 & 0,0 & 0,1 & 9,8 & 0,0 & 0,0 & 0,1 & 12,7 \\
\hline kein Verwertungsziel & 30 & 0,0 & 0 & 0,0 & 1 & 3,3 & 0 & 0,0 & 6 & 20,0 & 0 & 0,0 & 4 & 13,3 & 1 & 3,3 & 0 & 0,0 & 18 & 60,0 \\
\hline Gesamtzuschuss*) & 1,6 & 2,1 & 0,0 & 0,0 & 0,1 & 3,2 & 0,0 & 0,0 & 0,4 & 25,1 & 0,0 & 0,0 & 0,1 & 7,4 & 0,1 & 4,9 & 0,0 & 0,0 & 1,0 & 59,4 \\
\hline Darlehen*) & 0,2 & 0,6 & 0,0 & 0,0 & 0,0 & 0,0 & 0,0 & 0,0 & 0,0 & 0,0 & 0,0 & 0,0 & 0,0 & 0,0 & 0,1 & 70,7 & 0,0 & 0,0 & 0,1 & 29,3 \\
\hline
\end{tabular}

$\left.{ }^{*}\right)$ Beträge in Millionen Euro 


\begin{tabular}{|c|c|c|c|c|c|c|c|c|c|c|c|c|c|c|c|c|c|c|c|c|}
\hline & \multicolumn{2}{|c|}{ Insgesamt } & \multicolumn{2}{|c|}{ Burgenland } & \multicolumn{2}{|c|}{ Kärnten } & \multicolumn{2}{|c|}{$\begin{array}{l}\text { Niederöster- } \\
\text { reich }\end{array}$} & \multicolumn{2}{|c|}{$\begin{array}{l}\text { Oberöster- } \\
\text { reich }\end{array}$} & \multicolumn{2}{|c|}{ Salzburg } & \multicolumn{2}{|c|}{ Steiermark } & \multicolumn{2}{|c|}{ Tirol } & \multicolumn{2}{|c|}{ Vorarlberg } & \multicolumn{2}{|c|}{ Wien } \\
\hline & abs. & in \% & abs. & in $\%$ & abs. & in $\%$ & abs. & in $\%$ & abs. & in $\%$ & abs. & in $\%$ & abs. & in $\%$ & abs. & in $\%$ & abs. & in $\%$ & abs. & in \% \\
\hline $\begin{array}{l}\text { Kontakte entstanden bzw. } \\
\text { intensiviert }{ }^{\star} \text { ) }\end{array}$ & 425 & 78,1 & 3 & 0,7 & 19 & 4,5 & 55 & 12,9 & 86 & 20,2 & 22 & 5,2 & 84 & 19,8 & 20 & 4,7 & 16 & 3,8 & 120 & 28,2 \\
\hline nein $\left.^{\star}\right)$ & 88 & 16,2 & 2 & 2,3 & 4 & 4,5 & 8 & 9,1 & 17 & 19,3 & 2 & 2,3 & 18 & 20,5 & 5 & 5,7 & 5 & 5,7 & 27 & 30,7 \\
\hline weiß nicht *) & 31 & 5,7 & 0 & 0,0 & 2 & 6,5 & 2 & 6,5 & 4 & 12,9 & 2 & 6,5 & 5 & 16,1 & 3 & 9,7 & 1 & 3,2 & 12 & 38,7 \\
\hline
\end{tabular}

Tabelle 26 Frage 23: Wurden Kontakte nach Projektabschluss weitergeführt?

\begin{tabular}{|c|c|c|c|c|c|c|c|c|c|c|c|c|c|c|c|c|c|c|c|c|}
\hline & \multicolumn{2}{|c|}{ Insgesamt } & \multicolumn{2}{|c|}{ Burgenland } & \multicolumn{2}{|c|}{ Kärnten } & \multicolumn{2}{|c|}{$\begin{array}{l}\text { Niederöster- } \\
\text { reich }\end{array}$} & \multicolumn{2}{|c|}{$\begin{array}{l}\text { Oberöster- } \\
\text { reich }\end{array}$} & \multicolumn{2}{|c|}{ Salzburg } & \multicolumn{2}{|c|}{ Steiermark } & \multicolumn{2}{|c|}{ Tirol } & \multicolumn{2}{|c|}{ Vorarlberg } & \multicolumn{2}{|c|}{ Wien } \\
\hline & abs. & in $\%$ & abs. & in $\%$ & abs. & in $\%$ & abs. & in $\%$ & abs. & in $\%$ & abs. & in $\%$ & abs. & in $\%$ & abs. & in $\%$ & abs. & in $\%$ & abs. & in \% \\
\hline $\begin{array}{l}\text { In Folgeprojekten weiter- } \\
{\text { geführt }{ }^{*} \text { ) }}\end{array}$ & 283 & 67,2 & 3 & 1,1 & 13 & 4,6 & 37 & 13,1 & 58 & 20,5 & 11 & 3,9 & 59 & 20,8 & 16 & 5,7 & 11 & 3,9 & 75 & 26,5 \\
\hline in Publikationen weitergeführt *) & 77 & 18,3 & 1 & 1,3 & 6 & 7,8 & 9 & 11,7 & 15 & 19,5 & 2 & 2,6 & 10 & 13,0 & 7 & 9,1 & 1 & 1,3 & 26 & 33,8 \\
\hline $\begin{array}{l}\text { ohne konkrete Projekte weiter- } \\
\text { geführt }{ }^{*} \text { ) }\end{array}$ & 155 & 36,8 & 1 & 0,6 & 7 & 4,5 & 25 & 16,1 & 28 & 18,1 & 9 & 5,8 & 29 & 18,7 & 4 & 2,6 & 5 & 3,2 & 47 & 30,3 \\
\hline nicht weitergeführt*) & 36 & 8,6 & 0 & 0,0 & 0 & 0,0 & 5 & 13,9 & 8 & 22,2 & 2 & 5,6 & 6 & 16,7 & 2 & 5,6 & 1 & 2,8 & 12 & 33,3 \\
\hline
\end{tabular}

*) Mehrfachnennungen erlaubt. Die Prozentangaben beziehen sich auf die $\mathrm{N}$ im jeweiligen Bundesland 
Tabelle 27 Frage 7: Wurde das Projektziel aus technisch/wirtschaftlicher Sicht erreicht? Nach Beschäftigtengrößenklassen

\begin{tabular}{|c|c|c|c|c|c|c|c|c|c|c|c|c|c|c|c|c|}
\hline & \multicolumn{2}{|c|}{ Gesamtsumme } & \multicolumn{2}{|c|}{$\begin{array}{c}1 \text { bis } 20 \\
\text { Beschäftigte }\end{array}$} & \multicolumn{2}{|c|}{$\begin{array}{c}21 \text { bis } 50 \\
\text { Beschäftigte }\end{array}$} & \multicolumn{2}{|c|}{$\begin{array}{c}51 \text { bis } 100 \\
\text { Beschäftigte }\end{array}$} & \multicolumn{2}{|c|}{$\begin{array}{l}101 \text { bis } 250 \\
\text { Beschäftigte }\end{array}$} & \multicolumn{2}{|c|}{$\begin{array}{l}251 \text { bis } 500 \\
\text { Beschäftigte }\end{array}$} & \multicolumn{2}{|c|}{$\begin{array}{l}501 \text { bis } 1000 \\
\text { Beschäftigte }\end{array}$} & \multicolumn{2}{|c|}{$\begin{array}{c}\text { über } 1000 \\
\text { Beschäftigte }\end{array}$} \\
\hline & abs. & in $\%$ & abs. & in $\%$ & abs. & in $\%$ & abs. & in $\%$ & abs. & in $\%$ & abs. & in $\%$ & abs. & in $\%$ & abs. & in $\%$ \\
\hline $\begin{array}{l}\text { Projekte insge- } \\
\text { samt }\end{array}$ & 532 & 100,0 & 202 & 33,8 & 75 & 16,1 & 44 & 6,0 & 68 & 14,3 & 37 & 8,5 & 39 & 8,1 & 67 & 13,2 \\
\hline $\begin{array}{l}\text { Gesamtzu- } \\
\text { schuss }^{*} \text { ) }\end{array}$ & 73,9 & 100,0 & 17,5 & 23,7 & 10,7 & 14,5 & 4,1 & 5,5 & 10,9 & 14,7 & 7,4 & 10,0 & 6,2 & 8,4 & 17,1 & 23,2 \\
\hline Darlehen*) & 29,8 & 100,0 & 4,1 & 13,8 & 4,2 & 14,1 & 2,2 & 7,3 & 4,8 & 16,0 & 4,6 & 15,5 & 2,8 & 9,4 & 7,1 & 23,8 \\
\hline $\begin{array}{l}\text { technischer } \\
\text { Sicht }\end{array}$ & 465 & 87,4 & 180 & 38,7 & 62 & 13,3 & 31 & 6,7 & 60 & 12,9 & 35 & 7,5 & 36 & 7,7 & 61 & 13,1 \\
\hline $\begin{array}{l}\text { Gesamtzu- } \\
\text { schuss }^{*} \text { ) }\end{array}$ & 63,8 & 86,3 & 15,7 & 24,6 & 8,6 & 13,4 & 3,1 & 4,9 & 8,7 & 13,7 & 7,0 & 11,0 & 5,7 & 8,9 & 15,0 & 23,5 \\
\hline Darlehen*) & 26,1 & 35,4 & 3,6 & 13,6 & 3,7 & 14,3 & 1,6 & 6,0 & 4,2 & 16,0 & 4,1 & 15,7 & 2,8 & 10,7 & 6,2 & 23,7 \\
\hline $\begin{array}{l}\text { wirtschaftlicher } \\
\text { Sicht }\end{array}$ & 284 & 53,4 & 93 & 32,7 & 42 & 14,8 & 19 & 6,7 & 35 & 12,3 & 27 & 9,5 & 25 & 8,8 & 43 & 15,1 \\
\hline $\begin{array}{l}\text { Gesamtzu- } \\
\text { schuss }^{*} \text { ) }\end{array}$ & 44,1 & 59,7 & 8,0 & 18,1 & 7,8 & 17,7 & 2,3 & 5,1 & 5,3 & 12,0 & 4,6 & 10,5 & 4,3 & 9,8 & 11,8 & 26,8 \\
\hline Darlehen*) & 19,8 & 66,2 & 2,0 & 10,3 & 3,3 & 16,6 & 1,4 & 7,1 & 2,7 & 13,6 & 3,9 & 19,6 & 2,8 & 14,0 & 3,7 & 18,8 \\
\hline
\end{tabular}

*) Mehrfachnennungen erlaubt. Die Prozentangaben beziehen sich auf die N in der jeweiligen Größenklasse 
Tabelle 28 Frage 10: Werden die Projektergebnisse im Unternehmen wirtschaftlich verwertet? Nach Beschäftigtengrößenklassen

\begin{tabular}{|c|c|c|c|c|c|c|c|c|c|c|c|c|c|c|c|c|}
\hline & \multicolumn{2}{|c|}{ Gesamtsumme } & \multicolumn{2}{|c|}{$\begin{array}{c}1 \text { bis } 20 \\
\text { Beschäftigte }\end{array}$} & \multicolumn{2}{|c|}{$\begin{array}{c}21 \text { bis } 50 \\
\text { Beschäftigte }\end{array}$} & \multicolumn{2}{|c|}{$\begin{array}{c}51 \text { bis } 100 \\
\text { Beschäftigte }\end{array}$} & \multicolumn{2}{|c|}{$\begin{array}{c}101 \text { bis } 250 \\
\text { Beschäftigte }\end{array}$} & \multicolumn{2}{|c|}{$\begin{array}{l}251 \text { bis } 500 \\
\text { Beschäftigte }\end{array}$} & \multicolumn{2}{|c|}{$\begin{array}{l}501 \text { bis } 1000 \\
\text { Beschäftigte }\end{array}$} & \multicolumn{2}{|c|}{$\begin{array}{c}\text { über } 1000 \\
\text { Beschäftigte }\end{array}$} \\
\hline & abs. & in $\%$ & abs. & in \% & abs. & in $\%$ & abs. & in $\%$ & abs. & in \% & abs. & in $\%$ & abs. & in $\%$ & abs. & in $\%$ \\
\hline $\begin{array}{l}\text { Projekte insge- } \\
\text { samt }\end{array}$ & 532 & 100,0 & 202 & 33,8 & 75 & 16,1 & 44 & 6,0 & 68 & 14,3 & 37 & 8,5 & 39 & 8,1 & 67 & 13,2 \\
\hline Gesamtzuschuss*) & 73,9 & 100,0 & 17,5 & 23,7 & 10,7 & 14,5 & 4,1 & 5,5 & 10,9 & 14,7 & 7,4 & 10,0 & 6,2 & 8,4 & 17,1 & 23,2 \\
\hline Darlehen*) & 29,8 & 100,0 & 4,1 & 13,8 & 4,2 & 14,1 & 2,2 & 7,3 & 4,8 & 16,0 & 4,6 & 15,5 & 2,8 & 9,4 & 7,1 & 23,8 \\
\hline $\begin{array}{l}\text { werden wirtsch. } \\
\text { verwertet }\end{array}$ & 259 & 48,7 & 92 & 35,5 & 39 & 15,1 & 16 & 6,2 & 31 & 12,0 & 24 & 9,3 & 23 & 8,9 & 34 & 13,1 \\
\hline Gesamtzuschuss*) & 42,4 & 57,4 & 8,5 & 20,1 & 6,3 & 14,9 & 2,2 & 5,2 & 6,5 & 15,2 & 4,8 & 11,3 & 3,6 & 8,5 & 10,5 & 24,8 \\
\hline Darlehen*) & 19,9 & 66,6 & 3,1 & 15,6 & 3,4 & 17,0 & 1,5 & 7,4 & 3,2 & 16,3 & 2,9 & 14,5 & 2,4 & 12,1 & 3,4 & 17,1 \\
\hline $\begin{array}{l}\text { in Zukunft wirtsch. } \\
\text { verwertet }\end{array}$ & 89 & 16,7 & 37 & 41,6 & 11 & 12,4 & 4 & 4,5 & 13 & 14,6 & 2 & 2,2 & 6 & 6,7 & 16 & 18,0 \\
\hline Gesamtzuschuss*) & 13,6 & 18,5 & 4,0 & 29,5 & 1,9 & 13,8 & 0,2 & 1,7 & 1,0 & 7,4 & 0,6 & 4,3 & 1,5 & 11,0 & 4,4 & 32,3 \\
\hline Darlehen*) & 5,0 & 16,9 & 0,5 & 8,9 & 0,5 & 10,3 & 0,1 & 1,2 & 0,5 & 9,3 & 0,6 & 11,1 & 0,3 & 6,6 & 2,7 & 52,7 \\
\hline $\begin{array}{l}\text { Erkenntnis- } \\
\text { erweiterung }\end{array}$ & 131 & 24,6 & 44 & 33,6 & 19 & 14,5 & 16 & 12,2 & 21 & 16,0 & 10 & 7,6 & 7 & 5,3 & 14 & 10,7 \\
\hline 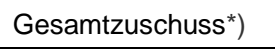 & 13,3 & 18,0 & 3,2 & 23,7 & 1,6 & 11,9 & 1,1 & 8,2 & 2,9 & 22,0 & 2,0 & 14,8 & 0,6 & 4,2 & 2,0 & 15,3 \\
\hline Darlehen*) & 4,2 & 13,9 & 0,3 & 8,3 & 0,3 & 7,4 & 0,5 & 12,6 & 0,8 & 19,0 & 1,2 & 28,9 & 0,1 & 1,7 & 0,9 & 22,1 \\
\hline keine Verwertung & 22 & 4,1 & 10 & 45,5 & 3 & 13,6 & 2 & 9,1 & 3 & 13,6 & 1 & 4,5 & 3 & 13,6 & 0 & 0,0 \\
\hline Gesamtzuschuss*) & 2,6 & 3,5 & 0,7 & 26,2 & 0,7 & 27,7 & 0,1 & 4,0 & 0,5 & 18,7 & 0,1 & 2,8 & 0,5 & 20,6 & 0,0 & 0,0 \\
\hline Darlehen $\left.^{\star}\right)$ & 0,6 & 0,8 & 0,2 & 38,8 & 0,0 & 0,0 & 0,1 & 12,7 & 0,3 & 48,5 & 0,0 & 0,0 & 0,0 & 0,0 & 0,0 & 0,0 \\
\hline $\begin{array}{l}\text { kein Verwertungs- } \\
\text { ziel }\end{array}$ & 29 & 5,5 & 18 & 62,1 & 2 & 6,9 & 6 & 20,7 & 0 & 0,0 & 0 & 0,0 & 0 & 0,0 & 3 & 10,3 \\
\hline Gesamtzuschuss*) & 1,6 & 2,1 & 0,9 & 57,5 & 0,1 & 4,5 & 0,4 & 28,4 & 0,0 & 0,0 & 0,0 & 0,0 & 0,0 & 0,0 & 0,1 & 9,5 \\
\hline Darlehen*) & 0,2 & 0,6 & 0,0 & 0,0 & 0,0 & 0,0 & 0,1 & 29,3 & 0,0 & 0,0 & 0,0 & 0,0 & 0,0 & 0,0 & 0,1 & 70,7 \\
\hline
\end{tabular}

*) Beträge in Millionen Euro 
Tabelle 29 Frage 22: Sind neue Kontakte/Kooperationen entstanden?

\begin{tabular}{|c|c|c|c|c|c|c|c|c|c|c|c|c|c|c|c|c|}
\hline & \multicolumn{2}{|c|}{ Insgesamt } & \multicolumn{2}{|c|}{$\begin{array}{c}1 \text { bis } 20 \\
\text { Beschäftigte }\end{array}$} & \multicolumn{2}{|c|}{$\begin{array}{c}21 \text { bis } 50 \\
\text { Beschäftigte }\end{array}$} & \multicolumn{2}{|c|}{$\begin{array}{l}51 \text { bis } 100 \\
\text { Beschäftigte }\end{array}$} & \multicolumn{2}{|c|}{$\begin{array}{c}101 \text { bis } 250 \\
\text { Beschäftigte }\end{array}$} & \multicolumn{2}{|c|}{$\begin{array}{l}251 \text { bis } 500 \\
\text { Beschäftigte }\end{array}$} & \multicolumn{2}{|c|}{$\begin{array}{l}501 \text { bis } 1000 \\
\text { Beschäftigte }\end{array}$} & \multicolumn{2}{|c|}{$\begin{array}{c}\text { über } 1000 \\
\text { Beschäftigte }\end{array}$} \\
\hline & abs. & in $\%$ & abs. & in $\%$ & abs. & in $\%$ & abs. & in $\%$ & abs. & in $\%$ & abs. & in $\%$ & abs. & in $\%$ & abs. & in $\%$ \\
\hline $\begin{array}{l}\text { Neue Kontakte ent- } \\
\text { standen }\end{array}$ & 410 & 77,7 & 146 & 35,6 & 57 & 13,9 & 35 & 8,5 & 51 & 12,4 & 29 & 7,1 & 33 & 8,0 & 59 & 14,4 \\
\hline nein & 87 & 16,5 & 41 & 47,1 & 12 & 13,8 & 8 & 9,2 & 13 & 14,9 & 5 & 5,7 & 5 & 5,7 & 3 & 3,4 \\
\hline weiß nicht & 31 & 5,9 & 12 & 38,7 & 5 & 16,1 & 1 & 3,2 & 4 & 12,9 & 3 & 9,7 & 1 & 3,2 & 5 & 16,1 \\
\hline
\end{tabular}

\section{Tabelle $30 \quad$ Frage 23: Wurden Kontakte nach Projektabschluss weitergeführt?}

\begin{tabular}{|c|c|c|c|c|c|c|c|c|c|c|c|c|c|c|c|c|}
\hline & \multicolumn{2}{|c|}{ Insgesamt } & \multicolumn{2}{|c|}{$\begin{array}{c}1 \text { bis } 20 \\
\text { Beschäftigte }\end{array}$} & \multicolumn{2}{|c|}{$\begin{array}{l}21 \text { bis } 50 \\
\text { Beschäftigte }\end{array}$} & \multicolumn{2}{|c|}{$\begin{array}{l}51 \text { bis } 100 \\
\text { Beschäftigte }\end{array}$} & \multicolumn{2}{|c|}{$\begin{array}{l}101 \text { bis } 250 \\
\text { Beschäftigte }\end{array}$} & \multicolumn{2}{|c|}{$\begin{array}{l}251 \text { bis } 500 \\
\text { Beschäftigte }\end{array}$} & \multicolumn{2}{|c|}{$\begin{array}{l}501 \text { bis } 1000 \\
\text { Beschäftigte }\end{array}$} & \multicolumn{2}{|c|}{$\begin{array}{l}\text { über } 1000 \\
\text { Beschäftigte }\end{array}$} \\
\hline & abs. & in $\%$ & abs. & in $\%$ & abs. & in $\%$ & abs. & in $\%$ & abs. & in $\%$ & abs. & in $\%$ & abs. & in \% & abs. & in $\%$ \\
\hline $\begin{array}{l}\text { In Folgeprojekten weiterge- } \\
\text { führt *) }\end{array}$ & 273 & 67,2 & 92 & 33,7 & 41 & 15,0 & 23 & 8,4 & 35 & 12,8 & 21 & 7,7 & 20 & 7,3 & 41 & 15,0 \\
\hline $\begin{array}{l}\text { in Publikationen weitergeführt } \\
\left.{ }^{*}\right)\end{array}$ & 76 & 18,7 & 32 & 42,1 & 15 & 19,7 & 2 & 2,6 & 5 & 6,6 & 2 & 2,6 & 5 & 6,6 & 15 & 19,7 \\
\hline $\begin{array}{l}\text { ohne konkrete Projekte wei- } \\
\text { tergeführt }{ }^{*} \text { ) }\end{array}$ & 152 & 37,4 & 63 & 41,4 & 19 & 12,5 & 13 & 8,6 & 16 & 10,5 & 11 & 7,2 & 11 & 7,2 & 19 & 12,5 \\
\hline nicht weitergeführt*) & 34 & 8,4 & 13 & 38,2 & 3 & 8,8 & 3 & 8,8 & 4 & 11,8 & 2 & 5,9 & 5 & 14,7 & 4 & 11,8 \\
\hline
\end{tabular}

*) Mehrfachnennungen erlaubt. Die Prozentangaben beziehen sich auf die N in der jeweiligen Größenklasse 
Tabelle $31 \quad$ Frage 7: Wurde das Projektziel aus technisch/wirtschaftlicher Sicht erreicht? Nach ÖNACE Klassifikation der Projekte

\begin{tabular}{|c|c|c|c|c|c|c|c|c|c|c|c|c|c|c|}
\hline & \multicolumn{2}{|c|}{ Gesamtsumme } & \multicolumn{2}{|c|}{ C } & \multicolumn{2}{|c|}{$\mathrm{D}, \mathrm{E}, \mathrm{F}$} & \multicolumn{2}{|c|}{ G,H } & \multicolumn{2}{|c|}{$\mathbf{J}$} & \multicolumn{2}{|c|}{ M } & \multicolumn{2}{|c|}{ Sonstige } \\
\hline & abs. & in $\%$ & abs. & in $\%$ & abs. & in \% & abs. & in $\%$ & abs. & in \% & abs. & in $\%$ & abs. & in \% \\
\hline Projekte insgesamt & 548 & $100,0 \%$ & 203 & $37,0 \%$ & 23 & $4,2 \%$ & 48 & $8,8 \%$ & 87 & $15,9 \%$ & 139 & $25,4 \%$ & 48 & $8,8 \%$ \\
\hline Gesamtzuschuss*) & 75,5 & $100,0 \%$ & 32,7 & $43,3 \%$ & 1,5 & $2,0 \%$ & 8,2 & $10,8 \%$ & 10,4 & $13,8 \%$ & 18,8 & $24,9 \%$ & 3,9 & $5,1 \%$ \\
\hline Darlehen*) & 30,2 & $100,0 \%$ & 16,3 & $54,0 \%$ & 0,7 & $2,4 \%$ & 4,7 & $15,4 \%$ & 3,0 & $9,9 \%$ & 5,2 & $17,2 \%$ & 0,3 & $1,1 \%$ \\
\hline technischer Sicht & 480 & $87,6 \%$ & 181 & $37,7 \%$ & 14 & $2,9 \%$ & 43 & $9,0 \%$ & 77 & $16,0 \%$ & 122 & $25,4 \%$ & 43 & $9,0 \%$ \\
\hline Gesamtzuschuss*) & 65,3 & $86,5 \%$ & 27,7 & $42,3 \%$ & 0,7 & $1,1 \%$ & 6,4 & $9,8 \%$ & 9,6 & $14,6 \%$ & 17,4 & $26,7 \%$ & 3,5 & $5,4 \%$ \\
\hline Darlehen*) & 26,5 & $87,8 \%$ & 14,8 & $55,6 \%$ & 0,1 & $0,3 \%$ & 3,8 & $14,3 \%$ & 2,7 & $10,0 \%$ & 5,0 & $18,8 \%$ & 0,3 & $1,1 \%$ \\
\hline wirtschaftlicher Sicht & 293 & $53,5 \%$ & 113 & $38,6 \%$ & 11 & $3,8 \%$ & 22 & $7,5 \%$ & 50 & $17,1 \%$ & 79 & $27,0 \%$ & 18 & $6,1 \%$ \\
\hline Gesamtzuschuss*) & 45,3 & $59,9 \%$ & 18,7 & $41,3 \%$ & 0,6 & $1,4 \%$ & 4,5 & $10,0 \%$ & 6,6 & $14,5 \%$ & 13,0 & $28,7 \%$ & 1,9 & $4,1 \%$ \\
\hline Darlehen*) & 20,1 & $66,4 \%$ & 11,4 & $56,6 \%$ & 0,0 & $0,1 \%$ & 1,6 & $7,8 \%$ & 2,3 & $11,2 \%$ & 4,5 & $22,6 \%$ & 0,3 & $1,7 \%$ \\
\hline
\end{tabular}

C: Herstellung von Waren

D:Abfallentsorgung/Beseitigung

E. Umweltverschmutzung: F. Bau
G: Handel, Instandhaltung und Reparatur von KFZ $\mathrm{H}$ : Verkehr und Lagerei

$\mathrm{J}$ : Information und Kommunikation

Sonstige, nicht eindeutig zuordenbare Projekte 
Tabelle 32 Frage 10: Werden die Projektergebnisse im Unternehmen wirtschaftlich verwertet? Nach ÖNACE Klassifikation der Projekte

\begin{tabular}{|c|c|c|c|c|c|c|c|c|c|c|c|c|c|c|}
\hline & \multicolumn{2}{|c|}{ Gesamtsumme } & \multicolumn{2}{|c|}{ C } & \multicolumn{2}{|c|}{$\mathrm{D}, \mathrm{E}, \mathrm{F}$} & \multicolumn{2}{|c|}{ G,H } & \multicolumn{2}{|c|}{ J } & \multicolumn{2}{|c|}{$\mathbf{M}$} & \multicolumn{2}{|c|}{ Sonstige } \\
\hline & abs. & in $\%$ & abs. & in $\%$ & abs. & in $\%$ & abs. & in $\%$ & abs. & in $\%$ & abs. & in $\%$ & abs. & in $\%$ \\
\hline Projekte insgesamt & 548 & $100,0 \%$ & 203 & $37,0 \%$ & 23 & $4,2 \%$ & 48 & $8,8 \%$ & 87 & $15,9 \%$ & 139 & $25,4 \%$ & 48 & $8,8 \%$ \\
\hline Gesamtzuschuss*) & 75,5 & $100,0 \%$ & 32,7 & $43,3 \%$ & 1,5 & $2,0 \%$ & 8,2 & $10,8 \%$ & 10,4 & $13,8 \%$ & 18,8 & $24,9 \%$ & 3,9 & $5,1 \%$ \\
\hline Darlehen*) & 30,2 & $100,0 \%$ & 16,3 & $54,0 \%$ & 0,7 & $2,4 \%$ & 4,7 & $15,4 \%$ & 3,0 & $9,9 \%$ & 5,2 & $17,2 \%$ & 0,3 & $1,1 \%$ \\
\hline $\begin{array}{l}\text { werden wirtsch. } \\
\text { verwertet }\end{array}$ & 266 & $48,5 \%$ & 113 & $42,5 \%$ & 10 & $3,8 \%$ & 20 & $7,5 \%$ & 47 & $17,7 \%$ & 60 & $22,6 \%$ & 16 & $6,0 \%$ \\
\hline Gesamtzuschuss*) & 43,6 & $57,7 \%$ & 20,3 & $46,5 \%$ & 0,7 & $1,6 \%$ & 4,4 & $10,0 \%$ & 6,4 & $14,8 \%$ & 10,6 & $24,3 \%$ & 1,2 & $2,7 \%$ \\
\hline Darlehen*) & 20,2 & $66,8 \%$ & 11,7 & $57,9 \%$ & 0,2 & $1,1 \%$ & 2,0 & $9,9 \%$ & 2,0 & $9,8 \%$ & 4,0 & $19,9 \%$ & 0,3 & $1,4 \%$ \\
\hline $\begin{array}{l}\text { in Zukunft wirtsch. } \\
\text { verwertet }\end{array}$ & 95 & $17,3 \%$ & 34 & $35,8 \%$ & 4 & $4,2 \%$ & 16 & $16,8 \%$ & 13 & $13,7 \%$ & 21 & $22,1 \%$ & 7 & $7,4 \%$ \\
\hline Gesamtzuschuss*) & 14,0 & $18,6 \%$ & 5,9 & $42,4 \%$ & 0,2 & $1,5 \%$ & 2,5 & $18,1 \%$ & 1,0 & $7,1 \%$ & 3,5 & $25,0 \%$ & 0,8 & $5,9 \%$ \\
\hline Darlehen*) & 5,1 & $16,9 \%$ & 2,4 & $47,8 \%$ & 0,0 & $0,0 \%$ & 1,7 & $32,4 \%$ & 0,3 & $5,1 \%$ & 0,8 & $14,7 \%$ & 0,0 & $0,0 \%$ \\
\hline Erkenntniserweiterung & 132 & $24,1 \%$ & 44 & $33,3 \%$ & 8 & $6,1 \%$ & 8 & $6,1 \%$ & 18 & $13,6 \%$ & 39 & $29,5 \%$ & 15 & $11,4 \%$ \\
\hline Gesamtzuschuss*) & 13,4 & $17,8 \%$ & 5,3 & $39,3 \%$ & 0,5 & $3,7 \%$ & 1,0 & $7,5 \%$ & 1,8 & $13,1 \%$ & 3,7 & $27,8 \%$ & 1,2 & $8,6 \%$ \\
\hline Darlehen*) & 4,2 & $13,7 \%$ & 2,0 & $49,2 \%$ & 0,2 & $5,5 \%$ & 0,9 & $22,6 \%$ & 0,6 & $13,4 \%$ & 0,3 & $8,2 \%$ & 0,0 & $1,1 \%$ \\
\hline keine Verwertung & 23 & $4,2 \%$ & 7 & $30,4 \%$ & 1 & $4,3 \%$ & 1 & $4,3 \%$ & 5 & $21,7 \%$ & 5 & $21,7 \%$ & 4 & $17,4 \%$ \\
\hline Gesamtzuschuss*) & 2,6 & $3,4 \%$ & 1,0 & $37,6 \%$ & 0,1 & $5,2 \%$ & 0,1 & $2,1 \%$ & 0,9 & $33,4 \%$ & 0,2 & $5,9 \%$ & 0,4 & $15,8 \%$ \\
\hline Darlehen*) & 0,6 & $0,7 \%$ & 0,0 & $2,6 \%$ & 0,3 & $48,5 \%$ & 0,1 & $9,8 \%$ & 0,1 & $22,4 \%$ & 0,1 & $16,7 \%$ & 0,0 & $0,0 \%$ \\
\hline kein Verwertungsziel & 30 & $5,5 \%$ & 5 & $16,7 \%$ & 0 & $0,0 \%$ & 3 & $10,0 \%$ & 3 & $10,0 \%$ & 13 & $43,3 \%$ & 6 & $20,0 \%$ \\
\hline Gesamtzuschuss*) & 1,6 & $3,7 \%$ & 0,3 & $16,4 \%$ & 0,0 & $0,0 \%$ & 0,2 & $13,0 \%$ & 0,2 & $12,8 \%$ & 0,7 & $40,7 \%$ & 0,3 & $17,1 \%$ \\
\hline Darlehen*) & 0,2 & $0,4 \%$ & 0,1 & $70,7 \%$ & 0,0 & $0,0 \%$ & 0,0 & $0,0 \%$ & 0,1 & $29,3 \%$ & 0,0 & $0,0 \%$ & 0,0 & $0,0 \%$ \\
\hline
\end{tabular}

C: Herstellung von Waren G: Handel, Instandhaltung und Reparatur von KFZ H: Verkehr und Lagerei

D:Abfallentsorgung/Beseitigung

$\mathrm{J}$ : Information und Kommunikation

M: Wissenschaftliche und technische Dienstleistungen

Sonstige, nicht eindeutig zuordenbare Projekte

$\mathrm{E}$ : Umweltverschmutzung; F: Bau 
Tabelle $33 \quad$ Frage 22: Sind neue Kontakte/Kooperationen entstanden?

\begin{tabular}{|c|c|c|c|c|c|c|c|c|c|c|c|c|c|c|}
\hline & \multicolumn{2}{|c|}{ Insgesamt } & \multicolumn{2}{|c|}{ C } & \multicolumn{2}{|c|}{$\mathrm{D}, \mathrm{E}, \mathrm{F}$} & \multicolumn{2}{|c|}{ G,H } & \multicolumn{2}{|c|}{$J$} & \multicolumn{2}{|c|}{$\mathbf{M}$} & \multicolumn{2}{|c|}{ Sonstige } \\
\hline & abs. & in $\%$ & abs. & in $\%$ & abs. & in $\%$ & abs. & in $\%$ & abs. & in $\%$ & abs. & in $\%$ & abs. & in $\%$ \\
\hline Neue Kontakte entstanden & 425 & $78,1 \%$ & 160 & $37,6 \%$ & 17 & $4,0 \%$ & 39 & $9,2 \%$ & 57 & $13,4 \%$ & 117 & $27,5 \%$ & 35 & $8,2 \%$ \\
\hline nein & 88 & $16,2 \%$ & 31 & $35,2 \%$ & 6 & $6,8 \%$ & 5 & $5,7 \%$ & 21 & $23,9 \%$ & 15 & $17,0 \%$ & 10 & $11,4 \%$ \\
\hline weiß nicht & 31 & $5,7 \%$ & 11 & $35,5 \%$ & 0 & $0,0 \%$ & 3 & $9,7 \%$ & 8 & $25,8 \%$ & 6 & $19,4 \%$ & 3 & $9,7 \%$ \\
\hline
\end{tabular}

C: Herstellung von Waren

G: Handel, Instandhaltung und Reparatur von KFZ

M: Wissenschaftliche und technische Dienstleistungen

D:Abfallentsorgung/Beseitigung $\mathrm{E}$ : Umweltverschmutzung; $\mathrm{F}$ : Bau $\mathrm{H}$ : Verkehr und Lagere

Sonstige, nicht eindeutig zuordenbare Projekte

Tabelle 34 Frage 23: Wurden Kontakte nach Projektabschluss weitergeführt?

\begin{tabular}{|c|c|c|c|c|c|c|c|c|c|c|c|c|c|c|}
\hline & \multicolumn{2}{|c|}{ Insgesamt } & \multicolumn{2}{|c|}{ C } & \multicolumn{2}{|c|}{$\mathrm{D}, \mathrm{E}, \mathrm{F}$} & \multicolumn{2}{|c|}{$\mathrm{G}, \mathrm{H}$} & \multicolumn{2}{|c|}{$\mathbf{J}$} & \multicolumn{2}{|c|}{$\mathbf{M}$} & \multicolumn{2}{|c|}{ Sonstige } \\
\hline & abs. & in $\%$ & abs. & in $\%$ & abs. & in \% & abs. & in $\%$ & abs. & in \% & abs. & in \% & abs. & in $\%$ \\
\hline In Folgeprojekten weitergeführt *) & 283 & $67,2 \%$ & 104 & $36,7 \%$ & 11 & $3,9 \%$ & 27 & $9,5 \%$ & 36 & $12,7 \%$ & 84 & $29,7 \%$ & 21 & $7,4 \%$ \\
\hline in Publikationen weitergeführt *) & 77 & $18,3 \%$ & 19 & $24,7 \%$ & 2 & $2,6 \%$ & 5 & $6,5 \%$ & 15 & $19,5 \%$ & 28 & $36,4 \%$ & 8 & $10,4 \%$ \\
\hline ohne konkrete Projekte weitergeführt *) & 155 & $36,8 \%$ & 59 & $38,1 \%$ & 8 & $5,2 \%$ & 15 & $9,7 \%$ & 19 & $12,3 \%$ & 42 & $27,1 \%$ & 12 & $7,7 \%$ \\
\hline nicht weitergeführt) & 36 & $8,6 \%$ & 16 & $44,4 \%$ & 1 & $2,8 \%$ & 1 & $2,8 \%$ & 4 & $11,1 \%$ & 8 & $22,2 \%$ & 6 & $16,7 \%$ \\
\hline
\end{tabular}

C: Herstellung von Waren

D:Abfallentsorgung/Beseitigung

E: Umweltverschmutzung: F: Bau
G: Handel, Instandhaltung und Reparatur von KFZ $\mathrm{H}$ : Verkehr und Lagerei

$\mathrm{J}$ : Information und Kommunikation
M: Wissenschaftliche und technische Dienstleistungen

Sonstige, nicht eindeutig zuordenbare Projekte 
KMU Forschung Austria

Austrian Institute for SME Research 